\title{
THE ALTERNATIVE TORUS AND THE STRUCTURE OF ELLIPTIC QUASI-SIMPLE LIE ALGEBRAS OF TYPE $A_{2}$
}

\author{
STEPHEN BERMAN, YUN GAO, YAROSLAV KRYLYUK, AND ERHARD NEHER \\ Dedicated to the memory of Professor Max Zorn
}

\begin{abstract}
We present the complete classification of the tame irreducible elliptic quasi-simple Lie algebras of type $A_{2}$, and in particular, specialize on the case where the coordinates are not associative. Here the coordinates are Cayley-Dickson algebras over Laurent polynomial rings in $\nu \geq 3$ variables, which we call alternative tori. In giving our classification we need to present much information on these alternative tori and the Lie algebras coordinatized by them.
\end{abstract}

\section{INTRODUCTION}

This paper is a follow-up to the work presented in [BGK] where a classification and realizations of certain simply-laced quasi-simple Lie algebras was carried out. In this paper we deal with the case where the core, $\mathscr{L}_{c}$, of the quasi-simple Lie algebra $\mathscr{L}$ over the complex field $\mathbb{C}$, is an $A_{2}$-graded Lie algebra. It is then the case that the core of $\mathscr{L}$ can be coordinatized by an alternative algebra with identity over $\mathbb{C}$. In the case when the alternative algebra is actually associative then it must be one of the quantum tori, $\mathbb{C}_{\mathbf{q}}$, studied in [BGK], and the algebras in question have the same structure and classification as those studied in [BGK], and, in fact, all of this follows as in that paper. There remains the intriguing possibility that the coordinates may be alternative but not associative, and then the question arises what these coordinate algebras may be, and what the structure of the attached quasi-simple Lie algebras is. This paper explicitly answers all of these questions by giving a classification and realizations of such algebras.

To be more specific recall that the concept of a quasi-simple Lie algebra was introduced in [H-KT] and, roughly speaking, this is a Lie algebra $\mathscr{L}$ over the complex field having an invariant, non-degenerate symmetric bilinear form

$$
(\cdot, \cdot): \mathscr{L} \times \mathscr{L} \rightarrow \mathbb{C},
$$

and a finite dimensional Cartan subalgebra $\mathscr{H} \subseteq \mathscr{L}$ so that the algebra has a

Received by the editors September 21, 1994.

1991 Mathematics Subject Classification. Primary 17B37, 17 B67.

The first and last authors gratefully acknowledge the support of the Natural Sciences and Engineering Research Council of Canada. 
root space decomposition

$$
\mathscr{L}=\bigoplus_{\alpha \in R} \mathscr{L}_{\alpha}
$$

where $\mathscr{L}_{\alpha}$ is defined in the usual way and $\mathscr{L}_{0}=\mathscr{H}$. Here $R$ is the root system and is to be discrete. One transfers the form to the dual space of $\mathscr{H}$ in the usual way and assumes that

$$
\operatorname{ad}_{\mathscr{L}} x \text { is nilpotent for } x \in \mathscr{L}_{\alpha} \text { when }(\alpha, \alpha) \neq 0,
$$

and that the form takes real values on the real span of the roots. $\mathscr{L}$ is said to be elliptic if the form is positive semi-definite on the real span of the roots. One attaches a finite root system, $\Delta$ (possibly non-reduced), to an elliptic $\mathscr{L}$ and says $\mathscr{L}$ is irreducible if $\Delta$ is irreducible and there are no isolated isotropic roots. One says that $\mathscr{L}$ is of type $X_{l}$ if $\Delta$ is the root system of type $X_{l}$. When $X_{l}$ is simply-laced of rank $l \geq 2$, so of the type $A_{l}, l \geq 2, D_{l}, l \geq 4$, or $E_{6}, E_{7}, E_{8}$, then the root system, $R$, of $\mathscr{L}$ is realized as $R=\Delta \times \Lambda$, where $\Lambda \cong \mathbb{Z}^{\nu}$ is a lattice of rank $\nu$. One says that $\nu$ is the nullity of $\mathscr{L}$ and it turns out for these simply-laced $\mathscr{L}$ that $\nu$ is an invariant of $\mathscr{L}$ since $\nu=\operatorname{dim}(\mathscr{L} /[\mathscr{L}, \mathscr{L}])$.

In [BGK] the simply-laced cases where $l \geq 3$ were dealt with. The method of attack is to define the core, $\mathscr{L}_{c}$, of $\mathscr{L}$ to be the subalgebra of $\mathscr{L}$ generated by the non-isotropic root spaces. It turns out that the core is an ideal of $\mathscr{L}$ so that one has the representation $\rho: \mathscr{L} \rightarrow$ End $\mathscr{L}_{c}$, induced by restricting the adjoint representation of $\mathscr{L}$ to $\mathscr{L}_{c}$. The core is always a $\Delta$-graded Lie algebra as defined in [BM] so in the simply-laced cases we use the coordinatization results there to give realizations of the core up to central extension. Already from these results it is apparent that only in the case where $\Delta$ is of type $A_{2}$ can one possibly have alternative, but not associative, coordinates. After realizing the core up to central extension one goes on to assume $\mathscr{L}$ is tame in the sense that $\operatorname{ker} \rho=\mathscr{Z}\left(\mathscr{L}_{c}\right)$, the center of the core. Then, using this and the known structure of the core one finds the algebra $\mathscr{L}$ roughly looks like a semi-direct product of $\mathscr{L}_{c}$ with an algebra of skew-symmetric outer derivations. Here "roughly" means there is a possible twisting by a 2-cocycle which is invariant and homogeneous in the sense of Definition 2.66. It is exactly this program that we carry out here for type $A_{2}$ tame, irreducible, elliptic quasi-simple Lie algebras with coordinates which are not associative. For simplicity we call such an algebra a QS algebra of type $A_{2}$.

If $\mathscr{L}$ is a $\mathrm{QS}$ algebra with core $\mathscr{L}_{c}$ then the coordinate algebra, $S$, of $\mathscr{L}_{c}$ must be an alternative algebra with identity $1 \in S$ over $\mathbb{C}$ having a $\mathbb{Z}^{\nu}$ gradation

$$
S=\bigoplus_{\mathbf{a} \in \mathbb{Z}^{\nu}} S_{\mathbf{a}}, \text { where } S_{0}=\mathbb{C} \cdot 1,
$$

and satisfying the rather strong properties that

$$
\begin{aligned}
& S_{\mathbf{a}} S_{\mathbf{b}}=S_{\mathbf{a}+\mathbf{b}}, \text { and } \\
& \operatorname{dim}_{\mathbb{C}} S_{\mathbf{a}}=1, \text { for all } \mathbf{a}, \mathbf{b} \in \mathbb{Z}^{\nu} .
\end{aligned}
$$

The quantum tori, $\mathbb{C}_{\mathbf{q}}$, are easily seen to be the only associative algebras with these properties (see (1.3) and Lemma 1.8). Moreover, if $\nu<3$ these are the only such algebras while there is one and only one such algebra $S$ which 
is not associative for each $\nu \geq 3$. We call these alternative tori and denote them by $\mathfrak{A}$. Once $\nu \geq 3$ is fixed, $\mathfrak{A}$ arises by three applications of the usual Cayley-Dickson process beginning with the commutative ring of Laurent polynomials $\mathbb{C}\left[t_{1}^{ \pm 1}, \ldots, t_{\nu}^{ \pm 1}\right]$ in $\nu$ variables. There are thus three choices of scalars, $\mu_{1}, \mu_{2}, \mu_{3}$, involved here and we always take $\mu_{i}=t_{i}, 1 \leq i \leq 3$. Thus, $\mathfrak{A}$ is an octonion or Cayley-Dickson algebra over the Laurent polynomial ring in $\nu$ variables.

In order to use $\mathfrak{A}$ as coordinates of a QS algebra $\mathscr{L}$ we need to understand some of its structural properties quite well. In particular, we need a full understanding of the derivation algebra $\operatorname{Der}_{\mathbb{C}}(\mathfrak{A})$. The Lie algebra $\operatorname{psl}_{\mathfrak{3}}(\mathfrak{A})$ can be most easily described as the quotient of the Steinberg Lie algebra $s_{3}(\mathfrak{A})$ with coordinates $\mathfrak{A}$ by its center. If $\mathscr{L}$ is a QS algebra with coordinates the alternative torus $\mathfrak{A}$ then there are surjective homomorphisms

$$
s t_{3}(\mathfrak{A}) \stackrel{\varphi}{\longrightarrow} \mathscr{L}_{c} \stackrel{\phi}{\longrightarrow} \operatorname{psl}_{3}(\mathfrak{A})
$$

with central kernels. In order to determine the structure of $\mathscr{L}$ from that of $\mathscr{L}_{c}$ it becomes important to understand the Schur multiplier $H_{2}\left(\operatorname{psl}_{3}(\mathfrak{A}), \mathbb{C}\right)=$ $\operatorname{ker}(\phi \circ \varphi)$. It turns out that this just depends on $\mathfrak{A}$, and in the associative cases one knows, by the results in [KL], that this is just the Connes first cyclic homology of $\mathfrak{A}$. We generalize all of this to the alternative case, and so define an analogue of this Connes first cyclic homology group for any alternative algebra and then go on to compute this in the case of the alternative torus. This is all we need here. It would be interesting to define $H C_{n}(S)$ for an alternative algebra $S$ and then, just as $H C_{n}\left(\mathbb{C}_{\mathbf{q}}\right)$ is computed in the recent paper [W], to compute $H C_{n}(\mathfrak{A})$.

The contents of the paper are as follows. In Section 1 we work with an alternative algebra and give our characterization of the alternative torus $\mathfrak{A}$. We then go on to study the derivation algebra of $\mathfrak{A}$ and the Connes first cyclic homology of $\mathfrak{A}$. For all of this we work over general fields $k$ of characteristic not 2 or 3 . The main results are Theorems 1.25, 1.40 and 1.64. In Section 2 we begin by showing how to obtain Lie algebras with coordinates an alternative algebra $S$, and we obtain such Lie algebras starting with any subspace $E$ of $H C_{1}(S)$. We also show that $H C_{1}(S)$ is the Schur multiplier of $p s l_{3}(S)$ by making use of the Steinberg Lie algebra $s t_{3}(S)$. We then study the spaces of invariant symmetric forms on these algebras and their derivation algebras, paying particular attention to the skew-symmetric derivations. The arguments about derivations resemble those in $[\mathrm{BGK}]$ and so we are brief here, referring the reader, for the most part, to that paper. Section 2 closes with a careful description of a class of Lie algebras, $L(\mathfrak{A}, \tau)$, which turn out to be all of the QS algebras with coordinates $\mathfrak{A}$. In particular, we show all such algebras have nondegenerate invariant symmetric bilinear forms. As in Section 1 we only need a base field $k$ of characteristic not 2 or 3 . In the third and final section we present our classification of the QS algebras of type $A_{2}$ having nonassociative coordinates. The arguments here are nearly identical with those in [BGK] and hence we are brief in our presentation. However, for the benefit of the reader, we have included the basic definitions and statements of the main theorems on the QS algebras from [H-KT] and [BGK] which we need, as well as the details necessary for our main theorem.

We feel that it is already clear that one can expect a complete classification 
of QS algebras of all types, even those of non-reduced type. Moreover, as QS algebras are very natural generalizations of both the finite dimensional simple Lie algebras and the affine Kac-Moody algebras (but include no other KacMoody algebras) one can hope for applications of this interesting class of Lie algebras. The works [BZ] and [N] present general coordinatization results in the non-simply-laced cases, generalizing those in [BM], and this is clearly the right starting point for classifying QS algebras. However, some of the coordinate algebras which arise seem quite complicated, and no doubt a classification of those with the necessary properties to be coordinates of QS algebras would be interesting in itself.

\section{The Alternative torus}

In this section we want to characterize the one alternative algebra over $\mathbb{C}$ which is not associative and which turns out to be the coordinates of some tame elliptic irreducible quasi-simple Lie algebras of type $A_{2}$. Although our main interest is in working over $\mathbb{C}$ we work over any field $k$ of characteristic not 2 at the outset since the restriction to $\mathbb{C}$ would not essentially shorten the presentation. We let $A_{[\nu]}$ (or $A_{[\nu]}(k)$ if reference to $k$ is necessary) be the ring of Laurent polynomials in the commuting variables $t_{1}, t_{2}, \ldots, t_{\nu}$ over $k$ so that

$$
A_{[\nu]}=k\left[t_{1}^{ \pm 1}, \ldots, t_{\nu}^{ \pm 1}\right]
$$

A variation of this leads to the so-called quantum torus, $k_{\mathbf{q}}$, associated to a $\nu \times \nu$ matrix $\mathbf{q}=\left(q_{i, j}\right)_{1 \leq i, j \leq \nu}$ of non-zero elements in $k$ satisfying

$$
q_{i, i}=1, \quad \text { and } \quad q_{i, j}=q_{j, i}^{-1}, \quad \text { for } \quad 1 \leq i, j \leq \nu .
$$

To define $k_{\mathbf{q}}$ one consideres the associative algebra $\tilde{A}_{[\nu]}$ of Laurent polynomials in the non-commuting variables $t_{1}, \ldots, t_{\nu}$ over $k$ and the 2-sided ideal

$$
I_{\mathbf{q}}=\left\langle t_{i} t_{j}-q_{i, j} t_{j} t_{i} \mid 1 \leq i, j \leq \nu\right\rangle \text {. }
$$

Then we define $k_{\mathbf{q}}$ by

$$
k_{\mathbf{q}}=\frac{\tilde{A}_{[\nu]}}{I_{\mathbf{q}}} .
$$

$k_{\mathbf{q}}$ is called the quantum torus associated to $\mathbf{q}$ (see [M]). Notice that $A_{[\nu]}$ is just $k_{1_{\nu}}$ where $1_{\nu}$ is the $\nu \times \nu$ matrix all of whose entries are 1 .

Let $\mathbb{Z}^{\nu}$ be the free abelian group of rank $\nu$. Then clearly $\tilde{A}_{[\nu]}$ has a $\mathbb{Z}^{\nu}$ gradation where $t_{i}$ is of degree $\epsilon_{i}=(0, \ldots, 0,1,0, \ldots, 0)$. Obviously $\epsilon_{i}$ is the vector with 1 in the $i^{\text {th }}$ place and zeros elsewhere. Moreover, $I_{\mathbf{q}}$ is homogeneous in this grading so that $k_{\mathbf{q}}$ is $\mathbb{Z}^{\nu}$ graded. Writing $A=k_{\mathbf{q}}$ we have

$$
A=\bigoplus_{\alpha \in \mathbb{Z}^{\nu}} A_{\alpha},
$$

with the following properties for $\alpha, \beta \in \mathbb{Z}^{\nu}$ and 1 the identity of $A$ :

$$
A_{\alpha} A_{\beta}=A_{\alpha+\beta}
$$

$$
\operatorname{dim} A_{\alpha}=1
$$


It then follows that

$$
A_{0}=k \cdot 1 \text {. }
$$

We let $t^{\alpha}=t_{1}^{\alpha_{1}} \cdots t_{\nu}^{\alpha_{\nu}}$ for $\alpha=\left(\alpha_{1}, \ldots, \alpha_{\nu}\right) \in \mathbb{Z}^{\nu}$ and have $A_{\alpha}=k \cdot t^{\alpha}$ for all $\alpha \in \mathbb{Z}^{\nu}$, so that $A$ has a basis consisting of monomials $t^{\alpha}, \alpha \in \mathbb{Z}^{\nu}$. It is clear that in such an algebra the monomials are units so that any non-zero homogeneous ideal is the whole algebra. Any such algebra is homogeneously simple, that is, has no non-trivial graded ideals. That the above properties characterize the associative algebras $k_{\mathbf{q}}$ is easy to see.

Lemma 1.8. Let $A$ be any $\mathbb{Z}^{\nu}$ graded associative algebra satisfying the above conditions (1.4), (1.5), (1.6), and (1.7). Then there is a $\nu \times \nu$ matrix of nonzero elements of $k, \mathbf{q}=\left(q_{i, j}\right)_{1 \leq i, j \leq \nu}$, satisfying $q_{i, i}=1, q_{i, j}=q_{j, i}^{-1}, 1 \leq$ $i, j \leq \nu$ such that $A$ is graded isomorphic to $k_{\mathbf{q}}$. Moreover, $A$ is commutative if and only if $\mathbf{q}=1_{\nu}$.

Proof. Clearly there is a surjective graded homomorphism $\phi: \tilde{A}_{\nu} \rightarrow A$ with $\phi(1)=1$ which is of degree 0 . Now $\phi\left(t_{i} t_{j}\right)=q_{i, j} \phi\left(t_{j} t_{i}\right)$ for some $q_{i, j} \in k$ since $\operatorname{dim} A_{\epsilon_{i}+\epsilon_{j}}=1$. Clearly $q_{i, i}=1$ and $q_{i, j} \neq 0$ because the monomials in $\tilde{A}_{[\nu]}$ are units and $\phi$ is non-zero on units. Then $0 \neq \phi\left(t_{i} t_{j}\right)=q_{i, j} \phi\left(t_{j} t_{i}\right)=$ $q_{i, j} q_{j, i} \phi\left(t_{i} t_{j}\right)$ implies that $q_{i, j} q_{j, i}=1$ so that $I_{\mathbf{q}}$ is contained in the kernel of $\phi$. But $k_{\mathbf{q}}$ is graded simple so that $I_{\mathbf{q}}=\operatorname{ker}(\phi)$, as desired.

We are interested in alternative algebras over $k$ which satisfy $(1.4),(1.5)$, $(1.6),(1.7)$. We first construct some algebras satisfying these properties and then go on to show that our construction yields all such algebras. For this we let $\nu \geq 3$ be fixed and consider

$$
K=A_{[\nu]}=k_{1_{\nu}}=k\left[t_{1}^{ \pm 1}, \ldots, t_{\nu}^{ \pm 1}\right],
$$

but this time with the grading by the abelian group $\Gamma=2 \mathbb{Z} \epsilon_{1} \oplus 2 \mathbb{Z} \epsilon_{2} \oplus 2 \mathbb{Z} \epsilon_{3} \oplus$ $\mathbb{Z} \epsilon_{4} \oplus \cdots \oplus \mathbb{Z} \epsilon_{\nu}$, where there are $\nu-3$ terms equal to $\mathbb{Z}$ and where

$$
\operatorname{deg}\left(t_{i}\right)=\left\{\begin{array}{rll}
2 \epsilon_{i} & \text { if } & 1 \leq i \leq 3, \\
\epsilon_{i} & \text { if } & 4 \leq i \leq \nu .
\end{array}\right.
$$

For any choice of non-zero scalars $s_{1}, s_{2}, s_{3} \in k$ let $A=A\left(K, s_{1} t_{1}, s_{2} t_{2}, s_{3} t_{3}\right)$ be the Cayley-Dickson algebra over the commutative ring $K$, which we consider as an algebra over $k$. We will see that $A$ has a $\mathbb{Z}^{\nu}$ gradation where (1.4), (1.5), (1.6), (1.7) hold.

We briefly recall this construction here. Towards this end let $B$ be any $K$ algebra with an identity 1 and with a $K$-linear involution $x \mapsto \bar{x}$ so that

$$
\left\{\begin{array}{c}
\overline{x y}=\bar{y} \bar{x} \quad \text { and }, \\
\overline{\bar{x}}=x \quad \text { for all } \quad x, y, \in B .
\end{array}\right.
$$

Then $\bar{a}=a$ for all $a \in K \cdot 1$. We assume that

$$
x \bar{x}=\bar{x} x \in K \cdot 1 \quad \text { for } \quad x \in B .
$$

Then

$$
x+\bar{x} \in K \cdot 1 \subseteq B
$$

follows. 
Notice that if we define $t$ and $n$ by $x+\bar{x}=t(x) \cdot 1$, and $x \bar{x}=\bar{x} x=n(x) \cdot 1$ for $x \in B$, then we have

$$
x^{2}-t(x) x+n(x) \cdot 1=0 \quad \text { for } \quad x \in B .
$$

Then $\overline{1}=1, t$ is $K$ linear, and $n$ is quadratic: $n(a \cdot 1)=a^{2}$ for $a \in K$,

$$
n(x, y):=n(x+y)-n(x)-n(y)=x \bar{y}+y \bar{x}
$$

is bilinear.

We now let $B^{\prime}$ be the algebra described as follows. $B^{\prime}=\left\{\left(b_{1}, b_{2}\right) \mid b_{1}, b_{2} \in\right.$ $B$ \} with addition and scalar multiplication by $K$ defined componentwise, and multiplication given by

$$
\left(b_{1}, c_{1}\right)\left(b_{2}, c_{2}\right)=\left(b_{1} b_{2}+\mu c_{2} \bar{c}_{1}, \bar{b}_{1} c_{2}+b_{2} c_{1}\right) \text {. }
$$

Here $\mu$ is choosen to be any element in $K$. Then $1=(1,0)$ is the identity of $B^{\prime}$, and if $j=(0,1)$ then $j^{2}=\mu \cdot 1$ and so we have

$$
B^{\prime}=B \oplus j B
$$

where we have identified $B$ as a subalgebra of $B^{\prime}$ by $b \leftrightarrow(b, 0)$.

Thus $B^{\prime}=\{b+j c \mid b, c \in B\}$ and multiplication is given by

$$
\left(b_{1}+j c_{1}\right)\left(b_{2}+j c_{2}\right)=\left(b_{1} b_{2}+\mu c_{2} \bar{c}_{1}\right)+j\left(\bar{b}_{1} c_{2}+b_{2} c_{1}\right) .
$$

We define an extension of our involution on $B$ by

$$
\overline{b+j c}=\bar{b}-j c,
$$

for all $b, c \in B$, and easily find that this is an involution on $B^{\prime}$. Also, we let $t, n$ be defined by $x+\bar{x}=t(x) \cdot 1=t(b) \cdot 1, n(x)=x \bar{x}(=\bar{x} x)=n(b)-\mu n(c)$, where $x=b+j c$, so that these maps also extend the corresponding ones on $B$.

We let $A_{0}=K$ and choose $\mu_{i}=s_{i} t_{i}, 1 \leq i \leq 3$, where $s_{i}$ is a non-zero element of $k$. Let $A_{i}=A_{i-1}^{\prime}$, for $1 \leq i \leq 3$ so that $A_{3}$ is the Cayley-Dickson algebra which we denoted before as $A\left(K, s_{1} t_{1}, s_{2} t_{2}, s_{3} t_{3}\right)$. Let

$$
\mathbb{Z}_{i}=\underbrace{\mathbb{Z} \oplus \cdots \oplus \mathbb{Z}}_{i \text {-times }} \oplus \underbrace{2 \mathbb{Z} \oplus \cdots \oplus 2 \mathbb{Z}}_{(3-i) \text {-times }} \oplus \mathbb{Z}^{\nu-3} \text {, for } i=0,1,2,3 .
$$

Note that $\mathbb{Z}_{0}=\Gamma$ so that $A_{0}=K$ has the grading which is specified in $(1.10)$, while we also have that $\mathbb{Z}_{3}=\mathbb{Z}^{\nu}$. We will show that each algebra $A_{i}$ has a grading by $\mathbb{Z}_{i}$ which satisfies properties analogous to (1.4) through (1.7) .

Lemma 1.20. With the notation as above we have the algebra $A_{i}$ has a grading by $\mathbb{Z}_{i}$ satisfying the following properties:

$$
A_{i}=\bigoplus_{\alpha \in \mathbb{Z}_{i}}\left(A_{i}\right)_{\alpha}
$$

where

$$
\begin{gathered}
\left(A_{i}\right)_{\alpha}\left(A_{i}\right)_{\beta}=\left(A_{i}\right)_{\alpha+\beta}, \\
\operatorname{dim}\left(A_{i}\right)_{\alpha}=1, \\
\left(A_{i}\right)_{0}=k \cdot 1
\end{gathered}
$$


for $\alpha, \beta \in \mathbb{Z}_{i}$ and where 1 is the identity of $A_{i}$. Moreover, the involution on $A_{i}$ satisfies $\bar{a}= \pm a$ for all $a \in\left(A_{i}\right)_{\alpha}$ and for all $\alpha \in \mathbb{Z}_{i}$.

Proof. We already know that $A_{0}$ has a gradation by $\mathbb{Z}_{0}$ satisfying the above properties (1)-(4) where we let $\bar{a}=a$ for all $a \in K=A_{0}$. By induction on $i$, if $A_{i-1}$ satisfies our requirements we simply let $\left(A_{i}\right)_{\alpha}=\left(A_{i-1}\right)_{\alpha}$ if $\alpha \in \mathbb{Z}_{i-1}$; while if $\alpha \in \mathbb{Z}_{i}$ but $\alpha \notin \mathbb{Z}_{i-1}$ we let $\left(A_{i}\right)_{\alpha}=j\left(A_{i-1}\right)_{\alpha-\epsilon_{i}}$. Then all properties are clear except possibly (2). For this consider the case where $\alpha \in \mathbb{Z}_{i-1}, \beta \in \mathbb{Z}_{i}$ but $\beta \notin \mathbb{Z}_{i-1}$. Let $\tilde{\beta}=\beta-\epsilon_{i}$ and let $a_{\alpha} \in\left(A_{i}\right)_{\alpha}, a_{\beta} \in\left(A_{i}\right)_{\beta}$, and say $e_{\tilde{\beta}} \in\left(A_{i-1}\right)_{\tilde{\beta}}$ satisfies $j e_{\tilde{\beta}}=a_{\beta}$. Then by $(1.17)$ we have $a_{\alpha} a_{\beta}=a_{\alpha} j e_{\tilde{\beta}}=$ $j\left(\bar{a}_{\alpha} e_{\tilde{\beta}}\right) \in\left(A_{i}\right)_{\alpha+\beta}$.

Next, consider the case where $\alpha, \beta \in \mathbb{Z}_{i}$ but $\alpha+\beta \notin \mathbb{Z}_{i-1}$, and use notation as above. We have by $(1.17)$ that $a_{\alpha} a_{\beta}=\left(j a_{\tilde{\alpha}}\right)\left(j a_{\tilde{\beta}}\right)$ so by our choice of scalars $\mu_{i}$ we get this equals $s_{i} t_{i} a_{\tilde{\beta}} \bar{a}_{\tilde{\alpha}}= \pm s_{i} t_{i} a_{\tilde{\beta}} a_{\tilde{\alpha}}$ which is in $\left(A_{i-1}\right)_{\alpha+\beta}$ since $\operatorname{deg}\left(t_{i}\right)=2 \epsilon_{i}$ by (1.10). The rest of the proof is obvious.

It is now clear that the algebra $A_{3}=A\left(K, s_{1} t_{1}, s_{2} t_{2}, s_{3} t_{3}\right)$ satisfies the properties (1.4), (1.5), (1.6), (1.7) and that this is an alternative algebra over $k$ which is not associative. We will denote this by $\mathfrak{A}$ or by $\mathfrak{A}\left(K, s_{1} t_{1}, s_{2} t_{2}, s_{3} t_{3}\right)$ if we need to refer to the scalars involved.

Definition 1.21. The algebra $\mathfrak{A}=\mathfrak{A}\left(K, s_{1} t_{1}, s_{2} t_{2}, s_{3} t_{3}\right)$ is called the alternative torus associated to the given scalars.

Remark 1.22. We recall here some basic facts on alternative and, in particular, octonion algebras. Let $\mathbb{O}$ be an octonion algebra over a ring $R$ without 2torsion. For $a, b, c \in \mathbb{O}$ we put

$$
[a, b]=a b-b a, \quad(a, b, c)=(a b) c-a(b c) .
$$

Since $\mathbb{O}$ is alternative, the nucleus $\mathscr{N}(\mathbb{O})$ of $\mathbb{O}$ is given by

$$
N(\mathbb{O})=\{x \in \mathbb{O}:(x, \mathbb{O}, \mathbb{O})=(0)\},
$$

while the center is

$$
\mathscr{Z}(\mathbb{O})=\{z \in N(\mathbb{O}):[z, \mathbb{O}]=(0)\} .
$$

Hence $R \cdot 1 \subseteq \mathscr{Z}(\mathbb{O}) \subseteq N(\mathbb{O})$. An easy calculation shows $N(\mathbb{O}) \subseteq R \cdot 1$, thus

$$
R \cdot 1=\mathscr{Z}(\mathbb{O})=N(\mathbb{O}) .
$$

We also put $\mathbb{O}^{\prime}=\{x \in \mathbb{O}: t(x)=0\}$ where $t$ is the trace map, and denote by $[\mathbb{O}, \mathbb{O}]($ resp. $(\mathbb{O}, \mathbb{O}, \mathbb{O})$ ) the span of all commutators (resp. associators) in $\mathbb{O}$. Then another calculation shows

$$
\mathbb{O}^{\prime}=[\mathbb{O}, \mathbb{O}]=(\mathbb{O}, \mathbb{O}, \mathbb{O}) .
$$

In particular, for $a, b, c \in \mathbb{O}$ we have

$$
t(a b)=t(b a), t((a, b, c))=0 .
$$

We now want to go towards our characterization of the alternative torus $\mathfrak{A}$. For this we need several lemmas. 
Lemma 1.23. Let $A$ be any alternative algebra over $k$ satisfying (1.4)-(1.7). Then

(i) $A$ is a domain in the sense that if $a, b \in A$ and $a b=0$ then either $a=0$ or $b=0$.

(ii) $A$ is prime in the sense that if $I, J$ are ideals of $A$ and $I J=(0)$, then either $I=(0)$ or $J=(0)$.

(iii) The center, $\mathscr{Z}(A)$, of $A$ is graded. In fact there is a subgroup $\Gamma_{\mathscr{Z}}$ of $\mathbb{Z}^{\nu}$ such that

$$
\mathscr{Z}(A)=\bigoplus_{\alpha \in \Gamma_{\mathscr{X}}} A_{\alpha}
$$

Proof. (i) is clear since $A$ is graded by $\mathbb{Z}^{\nu}$ and (1.5) also holds. (ii) follows immediately from (i). As for (iii) we see $\mathscr{Z}(A)$ is graded so that

$$
\mathscr{Z}(A)=\bigoplus_{\alpha \in \mathbb{Z}^{\nu}}\left(\mathscr{Z}(A) \cap A_{\alpha}\right),
$$

and either $\mathscr{Z}(A) \cap A_{\alpha}=(0)$ or $\mathscr{Z}(A) \cap A_{\alpha}=A_{\alpha}$ because of (1.6). Moreover, by (1.7) we have $A_{0}=k \cdot 1 \subseteq \mathscr{Z}(A)$ and $A_{\alpha} A_{-\alpha}=A_{0}=k \cdot 1$ so that $A_{\alpha} \subseteq \mathscr{Z}(A)$ if and only if $A_{-\alpha} \subseteq \mathscr{Z}(A)$. Thus, $\Gamma_{\mathscr{Z}}=\left\{\alpha \in \mathbb{Z}^{\nu} \mid A_{\alpha} \subseteq \mathscr{Z}(A)\right\}$ is a subgroup of $\mathbb{Z}^{\nu}$.

In the next lemma we consider a Cayley-Dickson algebra $\mathbb{O}$ over a field $k$ (we always assume the characteristic of $k$ is not 2$)$. Thus, $\mathbb{O}=A\left(k, \mu_{1}, \mu_{2}, \mu_{3}\right)$ for some choice of non-zero scalars $\mu_{1}, \mu_{2}, \mu_{3} \in k$. We have the norm and trace maps $n, t$ of $\mathbb{O} \rightarrow k$. We also have the bilinear form obtained from the quadratic form $n$ which we denote as $n(\cdot, \cdot): \mathbb{O} \times \mathbb{O} \rightarrow k$ defined by $n(x, y)=n(x+y)-n(x)-n(y)$ for all $x, y \in \mathbb{O}$.

Lemma 1.24. Let $\mathbb{O}$ be a Cayley-Dickson algebra over the field $k$ with identity element $1 \in \mathbb{O}$.

(i) If $x \in \mathbb{O}$ and $x \notin k \cdot 1$ then $x^{2} \in k \cdot 1 \Leftrightarrow t(x)=0 \Leftrightarrow \bar{x}=-x$.

(ii) Suppose $\mathbb{O}$ has a $(\mathbb{Z} / 2 \mathbb{Z})^{3}$ grading in the sense that $\mathbb{O}=\bigoplus_{\rho \in(\mathbb{Z} / 2 \mathbb{Z})^{3}} \mathbb{O}_{\rho}$ where $\mathbb{O}_{\rho} \mathbb{O}_{\mu} \subseteq \mathbb{O}_{\rho+\mu}, \mathbb{O}_{0}=k \cdot 1$ for $\rho, \mu \in(\mathbb{Z} / 2 \mathbb{Z})^{3}$. Then $n\left(\mathbb{O}_{\rho}, \mathbb{O}_{\mu}\right)=(0)$ if $\rho \neq \mu$.

Proof. We know that $x^{2}-t(x) x+n(x) \cdot 1=0$ for all $x \in \mathbb{O}$ so that $t(x)=0$ implies $x^{2}=-n(x) \cdot 1 \in k \cdot 1$. Also, letting $\mathbb{O}^{\prime}$ be the kernel of $t$ we have $\mathbb{O}=$ $k \cdot 1 \oplus \mathbb{O}^{\prime}$ so if $x=\alpha \cdot 1+x_{0}$ where $\alpha \in k, x_{0} \in \mathbb{O}^{\prime}$, then $x^{2}=\left(\alpha^{2} \cdot 1+x_{0}^{2}\right)+2 \alpha x_{0}$, where $\alpha^{2} \cdot 1+x_{0}^{2} \in k \cdot 1$ and $2 \alpha x_{0} \in \mathbb{O}^{\prime}$. Thus, $x^{2} \in k$ implies that $t(x)=0$. Also, for all $y \in \mathbb{O}$ we have $y+\bar{y}=t(y) \cdot 1$ so that $y+\bar{y}=0 \Leftrightarrow t(y)=0$. This proves (i).

As for (ii) note first that $\bar{x}=x$ for $x \in k \cdot 1$ and if $\rho \neq 0, x \in \mathbb{O}_{\rho}$ we have $x \notin k \cdot 1=\mathbb{O}_{0}$ but $x^{2} \in \mathbb{O}_{2 \rho}=\mathbb{O}_{0}=k \cdot 1$ so that by (i) $\bar{x}=-x$. For $x, y \in \mathbb{O}$ we have that

$$
n(x, y)=n(x+y)-n(x)-n(y)=x \bar{y}+y \bar{x},
$$

for $x, y \in \mathbb{O}$. Let $x \in \mathbb{O}_{\rho}, y \in \mathbb{O}_{\mu}$ with $\rho \neq \mu, \rho, \mu \in(\mathbb{Z} / 2 \mathbb{Z})^{3}$. If $\rho=0$ then $\bar{x}=x, \bar{y}=-y$ so $x \bar{y}+y \bar{x}=0$ as desired. By symmetry in $x$ and $y$ we may now assume $\mu \neq 0, \rho \neq 0$. Then $x \bar{y}+y \bar{x}=-(x y+y x)$. Now $\mu+\rho \neq 0$ since otherwise $\mu=-\rho=\rho$, so $x y \notin k \cdot 1=\mathbb{O}_{0}$. But $(x y)^{2} \in \mathbb{O}_{2(\mu+\rho)}=$ 
$\mathbb{O}_{0}=k \cdot 1$ so by (i) we get $\overline{x y}=-x y$ so $-x y=\bar{y} \bar{x}=(-y)(-x)=y x$ or $x y+y x=0$, giving us $n(x, y)=0$ as desired.

The following result is our main characterization of $\mathfrak{A}$.

Theorem 1.25. Let $A$ be any alternative algebra over $k$ satisfying (1.4)-(1.7) which is not associative. Then $A$ is isomorphic to an alternative torus of the form

$$
\mathfrak{A}\left(K, s_{1} t_{1}, s_{2} t_{2}, s_{3} t_{3}\right)
$$

for some choice of non-zero scalars $s_{i} \in k, 1 \leq i \leq 3$.

Proof. Note that by Lemma 1.23 and Lemma 1.8 the center of $A, \mathscr{Z}=\mathscr{Z}(A)$, is isomorphic to

$$
k\left[T_{1}^{ \pm 1}, \ldots, T_{m}^{ \pm 1}\right]
$$

for some variables $T_{1}, \ldots, T_{m}$, since every subgroup $\Gamma_{\mathscr{Z}}$ of $\mathbb{Z}^{\nu}$ is free of rank $m \leq \nu$. Moreover, letting $\mathscr{Z}^{*}$ denote the non-zero elements of $\mathscr{Z}$ and $\tilde{k}$ the quotient field we have $\left(\mathscr{Z}^{*}\right)^{-1} \mathscr{Z}=\tilde{k}$. Also, Lemma 1.23 shows that the hypothesis of Slater's Theorem holds for $A$ (see [SSSZ], p. 194) so since $A$ is not associative it is a Cayley-Dickson ring. Thus letting $\tilde{A}=\left(\mathscr{Z}^{*}\right)^{-1} A$ we have an embedding $A \hookrightarrow \tilde{A}$ where $\tilde{A}$ is a Cayley-Dickson algebra over the field $\tilde{k}$.

We are going to show that $\mathbb{Z}^{\nu} / \Gamma_{\mathscr{Z}} \cong(\mathbb{Z} / 2 \mathbb{Z})^{3}$, and to do this we introduce a new gradation on $A$ by the group $\Phi=\mathbb{Z}^{\nu} / \Gamma_{\mathscr{Z}}$. Let $\bar{\alpha}=\alpha+\Gamma_{\mathscr{Z}} \in \Phi$ and let

$$
A_{\overline{0}}=\mathscr{Z}(A), \quad A_{\bar{\alpha}}=\bigoplus_{\alpha \in \bar{\alpha}} A_{\alpha}, \text { for all } \bar{\alpha} \in \Phi \text {. }
$$

Thus $A=\bigoplus_{\bar{\alpha} \in \Phi} A_{\bar{\alpha}}$ and $A_{\bar{\alpha}} A_{\bar{\beta}}=A_{\bar{\alpha}+\bar{\beta}}, \mathscr{Z} A_{\bar{\alpha}}=A_{\bar{\alpha}}$ for all $\bar{\alpha} \in \Phi$. Next we introduce a $\Phi$-gradation on $\tilde{A}$ by saying $\tilde{A}_{\bar{\alpha}}=\left(\mathscr{Z}^{*}\right)^{-1} A_{\bar{\alpha}}$ so that $\tilde{A}_{0}=$ $\left(\mathscr{Z}^{*}\right)^{-1} \mathscr{Z}=\tilde{k}$. Clearly $\tilde{A}_{\bar{\alpha}}$ is a vector space over $\tilde{k}$ generated by any non-zero element $a_{\alpha} \in A_{\alpha}$ for $\alpha \in \bar{\alpha}$ so that $\tilde{A}_{\bar{\alpha}}$ is a 1-dimensional vector space over $\tilde{k}$. This implies that the order of $\Phi, o(\Phi)$, equals $8=\operatorname{dim}_{\tilde{k}} \tilde{A}$.

Next note that in the Cayley-Dickson algebra $\tilde{A}$ we have that for all $x \in A$

$$
x^{2}-t(x) x+n(x) \cdot 1=0,
$$

where $t$ is a $\tilde{k}$-linear and $n$ is a quadratic form on $\tilde{A}$ over $\tilde{k}$. Evaluating this for $x \in \tilde{A}_{\bar{\alpha}}, \bar{\alpha} \neq \overline{0}$, gives us

$$
x^{2}+n(x) \cdot 1=t(x) x \in \tilde{A}_{\bar{\alpha}} \cap\left(\tilde{A}_{2 \bar{\alpha}}+A_{\overline{0}}\right) .
$$

Now $\bar{\alpha} \neq 0$ implies $2 \bar{\alpha} \neq \bar{\alpha}$ so that $x^{2}+n(x) \cdot 1=t(x) x=0$. This implies that $x^{2}=-n(x) \cdot 1 \in \tilde{A}_{0} \cap \tilde{A}_{2 \bar{\alpha}}$ so we get that $2 \bar{\alpha}=0$. It follows that $\Phi \cong(\mathbb{Z} / 2 \mathbb{Z})^{3}$.

Now there is a basis $e_{1}, \ldots, e_{\nu}$ of $\mathbb{Z}^{\nu}$ and non-negative integers $d_{i} \geq 1$ with $d_{i} \mid d_{i-1}$ for $2 \leq i \leq \nu$ such that $\Gamma_{\mathscr{X}}=d_{1} \mathbb{Z} e_{1} \oplus \cdots \oplus d_{\nu} \mathbb{Z} e_{\nu}$. Since $\Phi=$ $\mathbb{Z}^{\nu} / \Gamma_{\mathscr{Z}} \cong(\mathbb{Z} / 2 \mathbb{Z})^{3}$ and since the elementary divisors $d_{1}, \ldots, d_{\nu}$ are uniquely determined by $\Gamma_{\mathscr{Z}}$ we find $d_{1}, d_{2}, d_{3}$ equal 2 and $d_{i}=1$ for $i \geq 4$. Thus, $\Gamma_{\mathscr{Z}} \cong \Gamma$. We now have $\nu \geq 3$ and and we can choose $0 \neq t_{i} \in A_{2 e_{i}}, 1 \leq i \leq 3$, $0 \neq t_{j} \in A_{e_{j}}, 4 \leq j \leq \nu$ such that

$$
K \cong k\left[T_{1}^{ \pm 1}, \ldots, T_{\nu}^{ \pm 1}\right] \rightarrow \mathscr{Z}(A)=k\left[t_{1}^{ \pm 1}, \ldots, t_{\nu}^{ \pm 1}\right]
$$

is an isomorphism of $\Gamma$-graded algebras where $K$ is given the $\Gamma$-grading as in (1.10). 
Next take $0 \neq a_{i} \in A_{e_{i}}, 1 \leq i \leq 3$ so we get $0 \neq a_{i}^{2} \in A_{2 e_{i}}=k t_{i}$ and thus there exists a non-zero element $s_{i} \in k$ such that $a_{i}^{2}=s_{i} t_{i}$. We know that $\tilde{A}$ is a Cayley-Dickson algebra over $\tilde{k}$ generated by $a_{1}, a_{2}, a_{3} \in A$. Moreover, it has as $\tilde{k}$ basis the elements $u_{1}=1, u_{2}=a_{1}, u_{3}=a_{2}, u_{4}=a_{1} a_{2}, u_{5}=a_{3}, u_{j+4}=$ $u_{j} u_{5}, 2 \leq j \leq 4$ and structure constants $\mu_{i}=s_{i} t_{i}, 1 \leq i \leq 3$, which are in $\mathscr{Z}(A)$. It now easily follows that $A$ is in fact a Cayley-Dickson algebra over the ring $\mathscr{Z}(A)$. To see this, we build up $\tilde{A}$ from $\tilde{k} 1$ using the Cayley-Dickson process. First, $a_{1} \in A_{e_{1}} \subseteq \tilde{A}_{\bar{e}_{1}}, \bar{e}_{1} \neq \overline{0}$, so $n\left(1, a_{1}\right)=0$ and we can use $a_{1}$ in the first step of the Cayley-Dickson process. Hence, the $\bar{k}$-subalgebra of $\tilde{A}$ generated by $a_{1}$ is $\tilde{k} 1 \oplus \tilde{k} a_{1}$ obtained by doubling $\tilde{k} 1$ with structure constant $s_{1} t_{1}$. Since $n\left(\tilde{k} 1 \oplus \tilde{k} a_{1}, a_{2}\right)=0$ by Lemma 1.24 (ii) and since $n$ is non-degenerate on $\tilde{k} 1 \oplus \tilde{k} a_{1}$ we can use $a_{2}$ in the next step of the CayleyDickson process. Repeating the process we can conclude that $A$ is a CayleyDickson algebra over the ring $\mathscr{Z}(A)$. So it is clear that $A$ has the elements $\left\{u_{1}, u_{2}, \ldots, u_{8}\right\}$ as a basis when considered as a free $\mathscr{Z}(A)$ module.

It is known that if $\mathfrak{A}=\mathfrak{A}\left(K, x_{1}, x_{2}, x_{3}\right)$ and $\mathfrak{A}^{\prime}=\mathfrak{A}\left(K, x_{1}^{\prime}, x_{2}^{\prime}, x_{3}^{\prime}\right)$ are Cayley-Dickson algebras such that $\frac{x_{i}}{x_{i}^{\prime}} \in\left(K^{*}\right)^{2}\left(K^{*}\right.$ is the non-zero element of $K$ ), then $\mathfrak{A} \cong \mathfrak{A}^{\prime}$. Thus, we have the following result.

Corollary 1.26. Let $A$ be an alternative algebra over the algebraically closed field $k$ which is not associative and satisfies (1.4)-(1.7). Then $A$ is isomorphic to $\mathfrak{A}=\mathfrak{A}\left(K, t_{1}, t_{2}, t_{3}\right)$, the alternative torus.

Our next goal is to obtain an understanding of the $k$-linear derivations of $\mathfrak{A}$, $\operatorname{Der}_{k}(\mathfrak{A})$, and the relationship of these to the $K$-linear derivations $\operatorname{Der}_{K}(\mathfrak{A})$, and the $k$-linear derivations of $K, \operatorname{Der}_{k}(K)$. In fact, we will show that we have a semi-direct product decomposition

$$
\operatorname{Der}_{k}(\mathfrak{A})=\operatorname{Der}_{K}(\mathfrak{A}) \oplus \operatorname{Der}_{k} K, \operatorname{Der}_{K}(\mathfrak{A}) \triangleleft \operatorname{Der}_{k}(\mathfrak{A})
$$

where $\operatorname{Der}_{k} K$ can be embedded in $\operatorname{Der}_{k}(\mathfrak{A})$ and turns out to be isomorphic to the outer $k$-derivations of $\mathfrak{A}$. These properties play an important role when we consider the first cyclic homology group of $\mathfrak{A}$ and construct quasi-simple algebras whose coordinates are the alternative torus $\mathfrak{A}$. Some of the techniques we use to study derivations of $\mathfrak{A}$ have appeared in other studies of derivations of Cayley-Dickson algebras or related algebras, and the reader can do no better than to consult $[\mathrm{McC}]$ for this and for further references on this point. Because derivations play such an important role in later developments we present most of the details necessary for the proofs, although we leave some of the more straightforward computations for the reader.

Remark 1.28. If $D \in \operatorname{Der}_{k}(\mathfrak{A})$, then, because $K$ equals the nucleus of $\mathfrak{A}$, we have, if $a \in K=N(\mathfrak{A})$,

$$
\begin{aligned}
0 & =D(0)=D((a, b, c)) \\
& =(D a, b, c)+(a, D b, c)+(a, b, D c)=(D a, b, c)
\end{aligned}
$$

for any $b, c \in \mathfrak{A}$ so that $D a \in K=N(\mathfrak{A})$. Thus, the restriction map $R$ : $\operatorname{Der}_{k}(\mathfrak{A}) \rightarrow \operatorname{Der}_{k}(K)$ defines a Lie algebra homomorphism whose kernel equals the ideal $\operatorname{Der}_{K}(\mathfrak{A})$ of the Lie algebra $\operatorname{Der}_{k}(\mathfrak{A})$. 
The Lie algebra of derivations, $\operatorname{Der}_{k}(K)$, is easy to understand. Towards this end we now introduce some notation. Recall that $K=k\left[t_{1}^{ \pm 1}, \ldots, t_{1}^{ \pm 1}\right]$ is $\Gamma=2 \mathbb{Z} \oplus 2 \mathbb{Z} \oplus 2 \mathbb{Z} \oplus \mathbb{Z}^{\nu-3}$ graded and that the alternative torus $\mathfrak{A}$ has a basis as a free $K$-module which we denote $u_{1}=1, u_{2}, \ldots, u_{8}$ and which satisfies the multiplication table on p. 5 of [S] where $\mu_{i}=s_{i} t_{i}$ for $1 \leq i \leq$ 3. Moreover, we usually identify $K$ with $K \cdot 1 \subseteq \mathfrak{A}$ and note that if we let $g_{1}=u_{2}, g_{2}=u_{3}$ and $g_{3}=u_{5}$, then $g_{i}^{2}=s_{i} t_{i}$ for $1 \leq i \leq 3$. As $g_{1}, g_{2}, g_{3}$ generated $\mathfrak{A}$ as a $K$-algebra then we find, letting $g_{j}=t_{j} \in K \subseteq \mathfrak{A}$ for $4 \leq j \leq \nu$, that $g_{1}^{ \pm 1}, \ldots, g_{\nu}^{ \pm 1}$ generate $\mathfrak{A}$ as a $k$-algebra. Also, $\operatorname{deg}\left(g_{i}\right)=\epsilon_{i}$ for $1 \leq i \leq \nu$. If $\mathbf{a}=\left(a_{1}, \ldots, a_{\nu}\right) \in \mathbb{Z}^{\nu}$ we define the element $g^{\mathbf{a}}$ by $g^{\mathbf{a}}=\left(\cdots\left(\left(g_{1}^{a_{1}} g_{2}^{a_{2}}\right) g_{3}^{a_{3}}\right) \cdots\right) g_{\nu}^{a_{\nu}}$. Then because (1.4)-(1.7) hold for $\mathfrak{A}$ we see that $g^{\mathfrak{a}}$ is a non-zero element in $\mathfrak{A}_{\mathbf{a}}$ so $\mathfrak{A}_{\mathfrak{a}}=k g^{\mathrm{a}}$. As $\mathfrak{A}$ is $\mathbb{Z}^{\nu}$-graded we have the $\nu$ degree derivations $d_{1}, \ldots, d_{\nu} \in \operatorname{Der}_{k}(\mathfrak{A})$ given by writing $d_{i}\left(g^{\mathbf{a}}\right)=a_{i} g^{\mathbf{a}}$ for any $\mathbf{a} \in \mathbb{Z}^{\nu}$. That is, $d_{i}$ acts as scalar multiplication by $a_{i}$ on $\mathfrak{A}_{\mathrm{a}}$, and in particular we have

$$
d_{i}\left(g_{j}\right)=\delta_{i j} g_{j}, \quad 1 \leq i, j \leq \nu .
$$

Moreover, it is very easy to see that if $D$ is any derivation of $\mathfrak{A}$ which is homogeneous of degree 0 then $D=\sum_{i=1}^{\nu} \alpha_{i} d_{i}$ for some scalars $\alpha_{1}, \ldots, \alpha_{\nu} \in$ $k$. We let

$$
\mathscr{D}_{0}=\left\{\sum_{i=1}^{\nu} \alpha_{i} d_{i} \mid \alpha_{i} \in k, 1 \leq i \leq \nu\right\} .
$$

Definition 1.31. For $\mathbf{a} \in \Gamma=2 \mathbb{Z} \oplus 2 \mathbb{Z} \oplus 2 \mathbb{Z} \oplus \mathbb{Z}^{\nu-3}$ and $\alpha \in k^{\nu}$ we define the map $\Gamma_{\mathbf{a}, \alpha}$ of $\mathfrak{A}$ to $\mathfrak{A}$ by

$$
\Gamma_{\mathbf{a}, \alpha}=g^{\mathbf{a}} \sum_{i=1}^{\nu} \alpha_{i} d_{i} .
$$

That is, $\Gamma_{\mathbf{a}, \alpha}\left(g^{\mathbf{b}}\right)=(\alpha \mid \mathbf{b}) g^{\mathbf{a}} g^{\mathbf{b}}$, where for any $\beta=\left(\beta_{1}, \ldots, \beta_{\nu}\right), \quad \gamma=$ $\left(\gamma_{1}, \ldots, \gamma_{\nu}\right)$ in $k^{\nu}$ we let $(\beta \mid \gamma)=\sum_{i=1}^{\nu} \beta_{i} \gamma_{i}$ be the usual dot product.

Since $K$ is the center of $\mathfrak{A}$ then if $\mathbf{a} \in \Gamma$ we know $\mathfrak{A}_{\mathfrak{a}}=k g^{\mathfrak{a}} \subseteq K$ so that $\Gamma_{\mathbf{a}, \alpha}$ is a derivation of $\mathfrak{A}$ which is in fact homogeneous of degree a because $\Gamma_{\mathbf{a}, \alpha}\left(\mathfrak{A}_{\mathbf{b}}\right) \subseteq \mathfrak{A}_{\mathbf{a}+\mathbf{b}}$ for any $\mathbf{b} \in \mathbb{Z}^{\nu}$. An easy computation leads to

$$
\left[\Gamma_{\mathbf{a}, \alpha}, \Gamma_{\mathbf{b}, \beta}\right]=\Gamma_{\mathbf{a}+\mathbf{b},(\alpha \mid \mathbf{b}) \beta-(\beta \mid \mathbf{a}) \alpha},
$$

for any $\mathbf{a}, \mathbf{b} \in \Gamma, \alpha, \beta \in k^{\nu}$.

It follows from this that the $k$-subspace $\mathscr{D}$ spanned by $\Gamma_{\mathbf{a}, \alpha}$ for $\mathbf{a} \in \Gamma, \alpha \in$ $k^{\nu}$ is a subalgebra of $\operatorname{Der}_{k}(\mathfrak{A})$. Also, we see that if $D=\sum_{\mathbf{a} \in \Gamma} \Gamma_{\mathbf{a}, \alpha(\mathbf{a})}$ is a derivation in $\mathscr{D}$, where $\alpha(\mathbf{a}) \in k^{\nu}$, which vanishes on $K$, then we have $D$ is identically zero on $\mathfrak{A}$. Indeed, if $i \in\{1,2,3\}$ then, as $a \in \Gamma$ implies $g^{\mathbf{a}}$ is central in $\mathfrak{A}$, we obtain from $D\left(g_{i}^{2}\right)=0$ that $2 g_{i} D\left(g_{i}\right)=0$ so that $g_{i}$ invertible implies that $D\left(g_{i}\right)=0$. It follows that $D$ is identically zero on $\mathfrak{A}$ and hence $R_{\mid \mathscr{D}}$, the restriction map $R$ from $\mathscr{D}$ to $\operatorname{Der}_{k}(K)$, is injective. We let $i: \operatorname{Der}_{k}(K) \rightarrow \operatorname{Der}_{k}(\mathfrak{A})$ be the inverse of $R_{\mid \mathscr{D}}$ and use this to identify $\operatorname{Der}_{k}(K)$ as a Lie subalgebra of $\operatorname{Der}_{k}(\mathfrak{A})$. It is a well-known fact that $\operatorname{Der}_{k}(K)=R(\mathscr{D})$ and so we now let $\mathscr{D}_{\mathbf{a}}=\left\{\Gamma_{\mathbf{a}, \alpha} \mid \alpha \in k^{\nu}\right\}$ for $\mathbf{a} \in \Gamma$, and use the above to identify $\operatorname{Der}_{k}(K)$ with $\mathscr{D}=\bigoplus_{\mathbf{a} \in \Gamma} \mathscr{D}_{\mathbf{a}}$. Thus, $i\left(\operatorname{Der}_{k}(K)\right)=\bigoplus_{\mathbf{a} \in \Gamma} \mathscr{D}_{\mathbf{a}}$. This leads us to the next result. 
Theorem 1.33. Let $\mathfrak{A}$ be an alternative torus over $k$. Then $\operatorname{Der}_{K}(\mathfrak{A})$ is an ideal of $\operatorname{Der}_{k}(\mathfrak{A})$ and we have the semi-direct product decomposition

$$
\operatorname{Der}_{k}(\mathfrak{A})=\operatorname{Der}_{K}(\mathfrak{A}) \oplus i\left(\operatorname{Der}_{k}(K)\right) .
$$

Proof. We have seen that $\operatorname{Der}_{K}(\mathfrak{A})$ is the kernel of $R$ and hence is an ideal of $\operatorname{Der}_{k}(\mathfrak{A})$. Moreover, if $D \in \operatorname{Der}_{k}(\mathfrak{A})$ then $D-i(R(D))$ is in the kernel of $R$ so $\operatorname{Der}_{k}(\mathfrak{A})=\operatorname{Der}_{K}(\mathfrak{A})+i\left(\operatorname{Der}_{k}(K)\right)$. Clearly the sum is direct.

Remark 1.34. A more general result than Theorem 1.33 is true. Let $\mathscr{C}$ be any Cayley-Dickson algebra, say $\mathscr{C}=\left(\mathscr{R}, \nu_{1}, \nu_{2}, \nu_{3}\right)$, over the commutative ring $\mathscr{R}$, where each $\nu_{i}$ is a non-zero invertible element in $\mathscr{R}$ and $\mathscr{R}$ is a $k$-algebra for some commutative ring $k$ :

(a) There is a unique Lie algebra homomorphism $i: \operatorname{Der}_{k}(\mathscr{R}) \rightarrow \operatorname{Der}_{k}(\mathscr{C})$ such that the restriction of $i(D)$ to $\mathscr{R}$ is just $D$ for $D \in \operatorname{Der}_{k}(\mathscr{R})$, and we have the semi-direct product decomposition

$$
\operatorname{Der}_{k}(\mathscr{C})=\operatorname{Der}_{\mathscr{R}}(\mathscr{C}) \oplus i\left(\operatorname{Der}_{k}(\mathscr{R})\right)
$$

(b) Let $1=u_{1}, u_{2}, \ldots, u_{8}$ be the standard $\mathscr{R}$ basis of $\mathscr{C}$ (see [S], p. 5) and let $g_{1}=u_{2}, g_{2}=u_{3}, g_{3}=u_{5}$ as above. Then for $D \in \operatorname{Der}_{k}(\mathscr{C})$ the following are equivalent:

(i) $D \in i\left(\operatorname{Der}_{k}(\mathscr{R})\right)$,

(ii) $D u_{i} \in \mathscr{R} u_{i}$ for $1 \leq i \leq 8$,

(iii) $D g_{i} \in \mathscr{R} g_{i}$ for $1 \leq i \leq 3$.

The proof of this, while not difficult, would take us too far afield of our main goal, and so is not included. Besides, the result in this generality is not needed for our later results.

We next investigate the inner derivations of $\mathfrak{A}$ and show they coincide with $\operatorname{Der}_{K}(\mathfrak{A})$. However, we work in more generality for a while and so let $\mathscr{S}$ be an alternative algebra over the commutative ring $\mathscr{R}$ of scalars. We assume $\mathscr{R}$ is an integral domain and its quotient field, $\mathscr{R}$, is not of characteristic 2 or 3 . Moreover, we assume $\mathscr{S}$ has an identity element $1 \in \mathscr{S}$ so $\mathscr{R}=\mathscr{R} \cdot 1 \subseteq \mathscr{S}$, with $\mathscr{R}$ in the center of $\mathscr{S}$.

Definition 1.35. Let $\mathscr{S}$ be as above and for $a \in \mathscr{S}$ we let $L_{a}$ (resp. $R_{a}$ ) denote the element in $\operatorname{End}_{\mathscr{R}}(\mathscr{S})$ denoting left (resp. right) multiplication on $\mathscr{S}$ by the element $a$. The inner derivation of $\mathscr{S}$ determined by the pair of elements $a, b \in \mathscr{S}$ is denoted $D_{a, b}$ and is defined by

$$
\begin{aligned}
D_{a, b} & =\left[L_{a}, L_{b}\right]+\left[L_{a}, R_{b}\right]+\left[R_{a}, R_{b}\right] \\
& =L_{[a, b]}-R_{[a, b]}-3\left[L_{a}, R_{b}\right] .
\end{aligned}
$$

Notice that the map $(a, b) \mapsto D_{a, b}$ is $\mathscr{R}$-bilinear. Moreover, it is a standard fact that $D_{a, b} \in \operatorname{Der}_{\mathscr{R}}(\mathscr{S})$, and that the following formulas hold. For references consult $[\mathrm{BK}]$ or $[\mathrm{S}]$.

$$
\begin{aligned}
& D_{a, b}+D_{b, a}=0, \\
& D_{a b, c}+D_{b c, a}+D_{c a, b}=0, \\
& {\left[D, D_{a, b}\right]=D_{D a, b}+D_{a, D b},}
\end{aligned}
$$

for any $a, b, c \in \mathscr{S}$, and any $D \in \operatorname{Der}_{\mathscr{R}}(\mathscr{S})$. 
Notice that it follows from these formulas and the definition that $D_{1, a}=$ 0 and $D_{a, a}=0$ for any $a \in \mathscr{S}$. We let $\operatorname{Inn}(\mathscr{S})$ denote the $\mathscr{R}$-span of all inner derivations $D_{a, b}$ for $a, b \in \mathscr{S}$. In our next result we let $\mathscr{S}=$ $\mathbb{O}$ be an octonian algebra over $\mathscr{R}$, so $\mathbb{O}$ is constructed from $\mathscr{R}$ by the 3 fold Cayley-Dickson process and is a free $\mathscr{R}$-module of rank 8 with a basis $u_{1}=1, u_{2}, \ldots, u_{8}$ satisfying the usual multiplication found in [S], p. 5 with structure constants $\mu_{1}, \mu_{2}, \mu_{3}$ being invertible elements in $\mathscr{R}$. It is a standard fact (see [BK] or [S]) that if $\mathscr{R}$ is a field of characteristic not 3 , then $\operatorname{Der}_{\mathscr{R}}(\mathbb{O})$ equals $\operatorname{Inn}(\mathbb{O})$ and that this is a 14-dimensional space. We will use this in our next result where we compare $\operatorname{Der}_{\mathscr{R}}(\mathbb{O}), \operatorname{Inn}(\mathbb{O})$ and the derivation algebra $\operatorname{Der}_{\mathscr{R}}(\tilde{\mathbb{O}})=\operatorname{Inn}(\tilde{\mathbb{O}})$ where $\tilde{\mathbb{O}}=\tilde{\mathscr{R}} \otimes_{\mathscr{R}} \mathbb{O}$ is the octonian algebra over the field $\tilde{\mathscr{R}}$ obtained from $\mathbb{O}$ by extending scalars.

Lemma 1.39. With the notation as above we have

(a) $\operatorname{Inn}(\mathbb{O})$ is a free $\mathscr{R}$-module of rank 14 .

(b) For every $D \in \operatorname{Der}_{\mathscr{R}}(\mathbb{O})$ there is a non-zero element $a \in \mathscr{R}$ such that $a D \in \operatorname{Inn}(\mathbb{O})$.

Proof. Since the map $(a, b) \mapsto D_{a, b} \in \operatorname{Inn}(\mathbb{C})$ is $\mathscr{R}$-bilinear and $D_{1, a}=0=$ $D_{a, a}$ for $a \in \mathbb{O}$ then $D_{a, b}=-D_{b, a}$ for $a, b \in \mathbb{O}$ implies $\operatorname{Inn}(\mathbb{O})$ is the $\mathscr{R}$-span of the 21 derivations $D_{u_{i}, u_{j}}=-D_{u_{j}, u_{i}}$ for $2 \leq i, j \leq 8, i \neq j$.

Next we use the relation (1.37), noting $u_{3} u_{2}=-u_{4}$, to get

$$
D_{-u_{4}, u_{i}}+D_{u_{2} u_{i}, u_{3}}+D_{u_{i} u_{3}, u_{2}}=0
$$

for $5 \leq i \leq 8$. Noting from the multiplication table of the $u$ 's that $\left\{u_{2} u_{i} \mid 5 \leq\right.$ $i \leq 8\}$ and $\left\{u_{i} u_{3} \mid 5 \leq i \leq 8\right\}$ both have $\mathscr{R}$-span equal to $\bigoplus_{i=5}^{8} \mathscr{R} u_{i}$, we see we can leave out the 4 derivations $D_{u_{j}, u_{2}}$ for $5 \leq j \leq 8$. Next note that $u_{5} u_{2}=u_{6}$ so

$$
D_{u_{6}, u_{i}}+D_{u_{2} u_{i}, u_{5}}+D_{u_{i} u_{5}, u_{2}}=0
$$

for $i=7,8$. Since the $\mathscr{R}$-span of $\left\{u_{2} u_{i} \mid i=7,8\right\}$ is $\bigoplus_{i=7}^{8} \mathscr{R} u_{i}$ and that of $\left\{u_{i} u_{5} \mid i=7,8\right\}$ is $\mathscr{R} u_{3} \oplus \mathscr{R} u_{4}$, we can get rid of two more derivations, namely $D_{u_{j}, u_{2}}$ for $j=3,4$. Finally, using the triple $u_{3}, u_{5}, u_{8}$ and noting $u_{3} u_{5}=-u_{7}, u_{5} u_{8}=\mu_{3} u_{4}, u_{8} u_{3}=\mu_{2} u_{6}$ give us

$$
D_{-u_{7}, u_{8}}+\mu_{3} D_{u_{4}, u_{3}}+\mu_{2} D_{u_{6}, u_{5}}=0
$$

so we can shed one more derivation, leaving us with the fact that $\operatorname{Inn}(\mathbb{O})$ is the $\mathscr{R}$-span of 14 derivations.

We note that any derivation $D \in \operatorname{Der}_{\mathscr{R}}(\mathbb{O})$ determines a derivation

$$
\tilde{D} \in \operatorname{Der}_{\tilde{R}}(\tilde{\mathbb{O}})=\operatorname{Inn}(\tilde{\mathbb{O}})
$$

given by $\tilde{D}(r \otimes u)=r \otimes D(u)$ for $r \in \mathscr{R}, u \in \mathbb{O}$. If $E$ is any inner derivation of $\tilde{\mathbb{O}}$, say $E=\sum_{2 \leq i<j \leq 8} r_{i j} D_{u_{i}, u_{j}}$ where $r_{i j} \in \tilde{\mathscr{R}}$ and we may suppose that $r_{i j}=\frac{s_{i j}}{s}$ for $s_{i j}, s \in \mathscr{R}$, then letting

$$
D=\sum_{2 \leq i<j \leq 8} s_{i j} D_{u_{i}, u_{j}} \in \operatorname{Inn}_{\mathscr{R}}(\mathbb{O})
$$

we find that $\frac{1}{s} \tilde{D}=E$. Thus, the map

$$
\lambda: \tilde{\mathscr{R}} \otimes_{\mathscr{R}} \operatorname{Inn}(\mathbb{O}) \rightarrow \operatorname{Inn}(\tilde{\mathbb{O}})
$$


given by $\lambda(r \otimes D)=r \tilde{D}$ for $r \in \tilde{\mathscr{R}}, D \in \operatorname{Inn}(\mathbb{O})$ is surjective, so that since we know $\operatorname{dim}_{\tilde{R}} \operatorname{Inn}(\tilde{\mathbb{O}})=14$ then we get $\lambda$ is an $\tilde{\mathscr{R}}$-linear isomorphism. It follows that $\operatorname{Inn}(\mathbb{O})$ is a free $\mathscr{R}$-module of rank 14 and this proves (a).

As for (b) we begin with $D \in \operatorname{Der}_{\mathscr{R}}(\mathbb{O})$ so that

$$
\tilde{D} \in \operatorname{Der}_{\tilde{R}}(\tilde{\mathbb{O}})=\operatorname{Inn}(\tilde{\mathbb{O}}) \cong \tilde{\mathscr{R}} \otimes \mathscr{R} \operatorname{Inn}(\mathbb{O}) \text {. }
$$

Then clearly, as above, there is some $E \in \operatorname{Inn}(\mathbb{O})$ and non-zero $a \in \mathscr{R}$ for which $\tilde{D}=\frac{1}{a} E$ so on $\mathbb{O}$ we get $a D=E$.

We next want to apply this to our alternative torus $\mathfrak{A}=\mathfrak{A}\left(K, s_{1} t_{1}, s_{2} t_{2}, s_{3} t_{3}\right)$ where $K=k\left[t_{1}^{ \pm 1}, \ldots, t_{1}^{ \pm 1}\right]$ with $\nu \geq 3$ and the characteristic of $k$ is not 2 or 3 . We know that $\mathfrak{A}$ is $\mathbb{Z}^{\nu}$-graded so for $\mathbf{a} \in \mathbb{Z}^{\nu}$ let $P_{\mathbf{a}}: \mathfrak{A} \rightarrow \mathfrak{A}_{\mathbf{a}}$ denote the projection afforded by this gradation. For $D \in \operatorname{Der}_{k}(\mathfrak{A})$ we see that if

$$
D_{\mathbf{a}}=\sum_{\mathbf{b} \in \mathbb{Z}^{\nu}} P_{\mathbf{a}+\mathbf{b}} \circ D \circ P_{\mathbf{b}}
$$

then each $D_{\mathbf{a}}$ is a derivation of $\mathfrak{A}$ and is homogeneous of degree a . Moreover since $\mathfrak{A}$ is generated as $k$-algebra by $g_{1}^{ \pm 1}, \ldots, g_{\nu}^{ \pm 1}$ then we see that the sum $\sum_{\mathbf{a} \in \mathbb{Z}^{\nu}} D_{\mathbf{a}}$ is finite and equals $D$. Thus, the derivation algebra, $\operatorname{Der}_{k}(\mathfrak{A})$, is $\mathbb{Z}^{\nu}$-graded and we let $\mathscr{D}_{\mathbf{a}}$ be the space of elements of degree a. Clearly both $\operatorname{Der}_{K}(\mathfrak{A})$ and $\operatorname{Inn}(\mathfrak{A})$ are graded subalgebras and we know $\operatorname{Inn}(\mathfrak{A}) \subseteq \operatorname{Der}_{K}(\mathfrak{A})$. If $D \in \operatorname{Der}_{K}(\mathfrak{A})$ is homogeneous of degree $\mathbf{a} \in \mathbb{Z}^{\nu}$, then by Lemma 1.43(b) there is some non-zero $r \in K$, for which $E=r D \in \operatorname{Inn}(\mathfrak{A})$. Writing $E=$ $\sum_{\mathbf{b} \in \mathbb{Z}^{\nu}} E_{\mathbf{b}}$, where $\bar{E}_{\mathbf{b}} \in \operatorname{Inn}(\mathfrak{A})$ is of degree $\mathbf{b}$ and $r=\sum_{\mathbf{b} \in \mathbb{Z}^{\nu}} r_{\mathbf{b}}$ where $r_{\mathbf{b}} \in K_{\mathbf{b}}$ is of degree $\mathbf{b}$, we find $r_{\mathbf{b}} D=E_{\mathbf{a}+\mathbf{b}}$ for all $\mathbf{b} \in \mathbb{Z}^{\nu}$. Now $r \neq 0$ implies $r_{\mathbf{b}} \neq 0$ for some $\mathbf{b} \in \mathbb{Z}^{\nu}$, so because $K_{\mathbf{b}}$ is a 1-dimensional $k$-vector space spanned by an invertible element in $K$ we obtain that $D=\frac{1}{r_{\mathbf{b}}} E_{\mathbf{a}+\mathbf{b}} \in \operatorname{Inn}(\mathfrak{A})$ so that $\operatorname{Inn}(\mathfrak{A})=\operatorname{Der}_{K}(\mathfrak{A})$. We can now state our main result on the derivation algebra $\operatorname{Der}_{k}(\mathfrak{A})$.

Theorem 1.40. Let $k$ be a field of characteristic not 2 or 3 and let

$$
\mathfrak{A}=\mathfrak{A}\left(K, s_{1} t_{1}, s_{2} t_{2}, s_{3} t_{3}\right)
$$

be an alternative torus. Then $\operatorname{Der}_{k}(\mathfrak{A})$ is $\mathbb{Z}^{\nu}$-graded so that

$$
\operatorname{Der}_{k}(\mathfrak{A})=\bigoplus_{\mathbf{a} \in \mathbb{Z}^{\nu}} \mathscr{D}_{\mathbf{a}}
$$

where $\mathscr{D}_{\mathbf{a}}$ is the space of derivations of degree a. We have

$$
\operatorname{Der}_{K}(\mathfrak{A})=\operatorname{Inn}(\mathfrak{A})=\bigoplus_{\mathbf{a} \in \mathbb{Z}^{\nu} \backslash \Gamma} \mathscr{D}_{\mathbf{a}}
$$

is an ideal of $\operatorname{Der}_{k}(\mathfrak{A})$ which is a free $K$-module of rank 14 while

$$
\operatorname{Der}_{k}(K)=\bigoplus_{\mathbf{a} \in \Gamma} \mathscr{D}_{\mathbf{a}}
$$

is a subalgebra of $\operatorname{Der}_{k}(K)$ which is isomorphic to the algebra of outer derivations of $\mathfrak{A}$. Moreover, if $\mathbf{a} \in \Gamma$, then $\mathscr{D}_{\mathbf{a}}=\left\{\Gamma_{\mathbf{a}, \alpha} \mid \alpha \in k^{\nu}\right\}$ is of dimension $\nu$, while if $\mathbf{a} \in \mathbb{Z}^{\nu} \backslash \Gamma$ then the dimension of the space $\mathscr{D}_{\mathbf{a}}$ equals 2 .

Proof. Recall $D_{1, a}=0=D_{a, a}$ for any $a \in K$ and $(a, b) \mapsto D_{a, b}$ is $K$ bilinear. Also, for $a, b \in \mathfrak{A}$ homogeneous and $r \in K$ homogeneous we have

$$
\operatorname{deg} r D_{a, b}=\operatorname{deg} r+\operatorname{deg} a+\operatorname{deg} b .
$$


We have seen that $\operatorname{Der}_{K}(\mathfrak{A})$ has a $K$ basis of elements of type $D_{u_{i}, u_{j}}$ for $2 \leq i \neq j \leq 8$ with $\operatorname{deg} u_{i}+\operatorname{deg} u_{j} \notin \Gamma$. Hence, any non-zero homogeneous element of $\operatorname{Inn}(\mathfrak{A})$ has its degree in $\mathbb{Z}^{\nu} \backslash \Gamma$. That is, we know $\operatorname{Der}_{K}(\mathfrak{A})=$ $\operatorname{Inn}(\mathfrak{A})=\bigoplus_{\mathbf{a} \in \mathbb{Z}^{\nu} \backslash \Gamma} \mathscr{D}_{\mathbf{a}}$.

Also, as $g^{\mathbf{b}} \mathscr{D}_{\mathbf{a}}=\mathscr{D}_{\mathbf{a}+\mathbf{b}}$ for $\mathbf{b} \in \Gamma$ (since $K=\bigoplus_{\mathbf{b} \in \Gamma} \mathfrak{A}_{\mathbf{b}}$ is the center of $\mathfrak{A}$ ), we need only compute the dimension of $\mathscr{D}_{\delta}$, for $\delta$ any representative of the 7 cosets of $\mathbb{Z}^{\nu} / \Gamma$ different from the coset $\Gamma$. Now $\mathbb{Z}^{\nu} / \Gamma$ is a direct sum of 3 cyclic groups of order 2 , so that if $\rho$ is any non-zero element of $\mathbb{Z}^{\nu} / \Gamma$ then there are exactly three 2-element subsets of $\mathbb{Z}^{\nu} / \Gamma$, say $\left\{\alpha_{i}, \beta_{i}\right\}$ for $i=1,2,3$, with $\alpha_{i}+\beta_{i}=\rho$. Then (1.37) leads to a non-trivial $K$-linear relation among the elements $D_{\alpha_{1}, \beta_{1}}, D_{\alpha_{2}, \beta_{2}}, D_{\alpha_{3}, \beta_{3}}$. But we know $\operatorname{Inn}(\mathfrak{A})$ is free of rank 14 as a $K$-module, so this implies that $\operatorname{dim} \mathscr{D}_{\mathbf{a}}=2$ for $\mathbf{a} \in \mathbb{Z}^{\nu} \backslash \Gamma$. The rest of the theorem has already been established.

We now want to discuss the analogue of the (Connes) first cyclic homology group of the alternative torus $\mathfrak{A}$. We begin by working with an arbitrary alternative algebra $S$ over the field $k$ which has an identity element $1 \in S$. Towards this end, let $I$ be the $k$-subspace of $S \otimes_{k} S$ spanned by the elements

$$
a \otimes b+b \otimes a, a b \otimes c+b c \otimes a+c a \otimes b,
$$

for all $a, b, c \in S$. Thus, we can form the factor space

$$
\langle S, S\rangle=\left(S \otimes_{k} S\right) / I .
$$

For notation we let $\langle a, b\rangle$ denote the coset $a \otimes b+I$ of $\langle S, S\rangle$. Then it follows that

$$
\begin{aligned}
& \langle a, b\rangle=-\langle b, a\rangle, \text { and } \\
& \langle a b, c\rangle+\langle b c, a\rangle+\langle c a, b\rangle=0,
\end{aligned}
$$

for all $a, b, c \in S$. Also, $\langle\cdot, \cdot\rangle$ is $k$-bilinear. It follows from (1.43) and (1.44) that $\langle 1, a\rangle=\langle a, 1\rangle=0$ and $\langle a, a\rangle=0$ for all $a \in S$. Because of (1.36), (1.37) there exists a linear map $\rho:\langle S, S\rangle \rightarrow \operatorname{Inn}(S)$ such that $\rho(\langle a, b\rangle)=D_{a, b}$.

Definition 1.45. We define the Connes first cyclic homology group of $S$, denoted $H C_{1}(S)$, by

$$
H C_{1}(S)=\left\{\sum_{i \in \mathscr{I}}\left\langle a_{i}, b_{i}\right\rangle \in\langle S, S\rangle \mid \sum_{i \in \mathcal{I}} D_{a_{i}, b_{i}}=0\right\}=\operatorname{ker} \rho,
$$

where $\mathscr{I}$ is a finite index set.

The reason for the name is that in the case $S$ is associative with $[S, S] \cap$ $\mathscr{Z}(S)=(0), H C_{1}(S)$ is indeed the usual Connes first cyclic homology group of $S$, see [BGK] or [KL].

Our goal is to see that $H C_{1}(S)$ is $\mathbb{Z}^{\nu}$ graded when $S=\mathfrak{A}$ and to compute the dimensions of the homogeneous spaces. This will be needed when we build quasi-simple algebras from $\mathfrak{A}$. Moreover, we will need some properties concerning $k$-bilinear maps satisfying identities like (1.43) and (1.44) so our next result concerns itself with this. 
Lemma 1.46. Let $\mathscr{E}$ be a $k$-vector space and $\{\cdot, \cdot\}: S \otimes_{k} S \rightarrow \mathscr{E}$ a $k$-bilinear mapping satisfying

$$
\begin{aligned}
& \{a, b\}+\{b, a\}=0, \text { and } \\
& \{a b, c\}+\{b c, a\}+\{c a, b\}=0,
\end{aligned}
$$

for all $a, b, c \in S$. Let $(a, b, c)=(a b) c-a(b c)$ be the associator of the ordered triple $a, b, c$ of elements in $S$. Then we have

$$
\{(a, b, c), d\}+\{c,(a, b, d)\}+\{(c, d, a), b\}+\{a,(c, d, b)\}=0,
$$

and

$$
\left\{D_{a, b} c, d\right\}+\left\{c, D_{a, b} d\right\}+\left\{D_{c, d} a, b\right\}+\left\{a, D_{c, d} b\right\}=0,
$$

for all $a, b, c, d \in S$. Moreover, let $\mathscr{E}^{\prime}$ be the $k$-span of all elements $\{a, b\} \in$ $\mathscr{E}$ for all $a, b \in S$. If there is a $k$-linear map $\pi: \mathscr{E}^{\prime} \rightarrow \operatorname{Inn}(S)$ satisfying $\pi(\{a, b\})=D_{a, b}$, then defining $[\cdot, \cdot]: \mathscr{E}^{\prime} \otimes_{k} \mathscr{E}^{\prime} \rightarrow \mathscr{E}^{\prime}$ as the $k$-bilinear extension of $[\{a, b\},\{c, d\}]=\left\{D_{a, b} c, d\right\}+\left\{c, D_{a, b} d\right\}$, we get this is a well-defined $k$-bilinear map making $\mathscr{E}^{\prime}$ into a Lie algebra in such a way that $\pi$ is a surjective Lie algebra homomorphism.

Proof. Recalling that in an alternative algebra the associator is an alternating function of its variables, we see the left-hand side in (1) is

$$
\{(a, b, c), d\}+\{c,(d, a, b)\}+\{(c, d, a), b\}+\{a,(b, c, d)\} .
$$

Expanding this and rearranging terms gives us

$$
\begin{aligned}
& \{(a b) c, d\}-\{c, d(a b)\}-\{a(b c), d\}+\{a,(b c) d\} \\
& \quad+\{c,(d a) b\}-\{c(d a), b\}+\{(c d) a, b\}-\{a, b(c d)\},
\end{aligned}
$$

which equals

$$
\begin{aligned}
& (\{(a b) c, d\}+\{d(a b), c\})-(\{a(b c), d\}+\{(b c) d, a\}) \\
& \quad-(\{(d a) b, c\}+\{c(d a), b\})+(\{(c d) a, b\}+\{b(c d), a\}) .
\end{aligned}
$$

Now by (ii) this is

$$
-\{c d, a b\}+\{d a, b c\}+\{b c, d a\}-\{a b, c d\}
$$

which is 0 by (i). This proves ( 1 ).

Expand the left-hand side of (2) using Definition 1.39 and rearrange terms to get

$$
\begin{aligned}
& \{(a b-b a) c, d\}-\{c, d(a b-b a)\}-\{c(a b-b a), d\}+\{c,(a b-b a) d\} \\
& \quad+\{(c d-d c) a, b\}-\{a, b(c d-d c)\}-\{a(c d-d c), b\}+\{a,(c d-d c) b\} \\
& \quad-3\{(a, b, c), d\}-3\{c,(a, b, d)\}-3\{(c, d, a), b\}-3\{a,(c, d, b)\} .
\end{aligned}
$$

Using (1) and (i) this becomes

$$
\begin{aligned}
& (\{(a b-b a) c, d\}+\{d(a b-b a), c\})-(\{c(a b-b a), d\}+\{(a b-b a) c, d\}) \\
& \quad+(\{(c d-d c) a, b\}+\{b(c d-d c), a\}) \\
& \quad-(\{a(c d-d c), b\}+\{(c d-d c) b, a\}) .
\end{aligned}
$$

By (ii) this is

$$
\{-c d, a b-b a\}+\{d c, a b-b a\}-\{a b, c d-d c\}+\{b a, c d-d c\},
$$

which is 0 by (i). This proves (2). 
The hypothesis and $(2)$ insure that $[\cdot, \cdot]$ is well-defined and anti-commutative. As for the Jacobi identity, let $a, b, c, d, e, f \in S$. Then

$$
\begin{aligned}
{[[\{a, b\},\{c, d\}],\{e, f\}]+[[\{c, d\},\{e, f\}],\{a, b\}] } \\
+[[\{e, f\},\{a, b\}],\{c, d\}] \\
=[[\{a, b\},\{c, d\}],\{e, f\}]-[\{a, b\},[\{c, d\},\{e, f\}]] \\
\quad+[\{c, d\},[\{a, b\},\{e, f\}]] .
\end{aligned}
$$

By definition this equals

$$
\begin{aligned}
{\left[\left\{D_{a, b} c, d\right\}+\left\{c, D_{a, b} d\right\},\{e, f\}\right] } \\
-\left[\{a, b\},\left\{D_{c, d} e, f\right\}+\left\{e, D_{c, d} f\right\}\right] \\
+\left[\{c, d\},\left\{D_{a, b} e, f\right\}+\left\{e, D_{a, b} f\right\}\right] .
\end{aligned}
$$

Using our definition of the bracket again and (1.38) this becomes

$$
\begin{aligned}
&\left\{D_{D_{a, b} c, d} e, f\right\}+\left\{e, D_{D_{a, b} c, d} f\right\}+\left\{D_{c, D_{a, b} d} e, f\right\}+\left\{e, D_{c, D_{a, b} d} f\right\} \\
&-\left\{D_{a, b}\left(D_{c, d} e\right), f\right\}-\left\{D_{c, d} e, D_{a, b} f\right\}-\left\{D_{a, b} e, D_{c, d} f\right\} \\
&-\left\{e, D_{a, b}\left(D_{c, d} f\right)\right\}+\left\{D_{c, d}\left(D_{a, b} e\right), f\right\} \\
&+\left\{D_{a, b} e, D_{c, d} f\right\}+\left\{D_{c, d} e, D_{a, b} f\right\}+\left\{e, D_{c, d}\left(D_{a, b} f\right)\right\} \\
&=\left\{D_{D_{a, b} c, d} e+D_{c, D_{a, b} d} e, f\right\}+\left\{e, D_{D_{a, b} c, d} f+D_{c, D_{a, b}} f\right\} \\
&-\left\{D_{a, b}\left(D_{c, d} e\right)-D_{c, d}\left(D_{a, b} e\right), f\right\}-\left\{e, D_{a, b}\left(D_{c, d} f\right)-D_{c, d}\left(D_{a, b} f\right)\right\} \\
&=\left\{\left[D_{a, b}, D_{c, d}\right] e, f\right\}+\left\{e,\left[D_{a, b}, D_{c, d}\right] f\right\} \\
&-\left\{\left[D_{a, b}, D_{c, d}\right] e, f\right\}-\left\{e,\left[D_{a, b}, D_{c, d}\right] f\right\} \\
&= 0 .
\end{aligned}
$$

Note that if $D \in \operatorname{Der}_{k}(S)$ then $D$ acts on $S \otimes_{k} S$ by linear extension of $D(a \otimes b)=D(a) \otimes b+a \otimes D b$. It is clear that the subspace $I$ defined as the $k$-span of the elements in (1.41) is mapped to itself by $D$ so that $D(I) \subseteq I$ and we have an induced action on the quotient space $\langle S, S\rangle$ satisfying

$$
D(\langle a, b\rangle)=\langle D a, b\rangle+\langle a, D b\rangle,
$$

for all $a, b \in S$.

Lemma 1.48. (1) If $D \in \operatorname{Der}_{k}(S)$ then $D$ induces an action on $H C_{1}(S)$ in the sense that $D\left(H C_{1}(S)\right) \subseteq H C_{1}(S)$.

(2) If $D \in \operatorname{Inn}(S)$, then $D$ acts trivially, that is as 0 , on $H C_{1}(S)$.

Proof. Recall the map $\rho:\langle S, S\rangle \rightarrow \operatorname{Inn}(S)$ given by $\rho(\langle a, b\rangle)=D_{a, b}$. Since $H C_{1}(S)=\operatorname{ker} \rho$ it suffices to prove that $\rho$ is a $\operatorname{Der}_{k}(S)$-module homomorphism. In fact, we have

$$
\begin{aligned}
\rho(D\langle a, b\rangle) & =\rho(\langle D a, b\rangle+\langle a, D b\rangle \\
& =D_{D a, b}+D_{a, D b}=\left[D, D_{a, b}\right]
\end{aligned}
$$

for $a, b \in S, D \in \operatorname{Der}_{k}(S)$.

Notice that $\langle\cdot, \cdot\rangle$ satisfies (i) and (ii) of Lemma 1.46 so by (2) of that lemma we have

$$
\begin{aligned}
D_{a, b}(\langle c, d\rangle) & =\left\langle D_{a, b} c, d\right\rangle+\left\langle c, D_{a, b} d\right\rangle \\
& =-\left(\left\langle D_{c, d} a, b\right\rangle+\left\langle a, D_{c, d} b\right\rangle\right)=-D_{c, d}(\langle a, b\rangle) .
\end{aligned}
$$


Thus, if we have $\sum_{i \in \mathcal{I}} D_{a_{i}, b_{i}}=0$, then

$$
\begin{aligned}
D_{c, d}\left(\sum_{i \in \mathscr{I}}\left\langle a_{i}, b_{i}\right\rangle\right) & =\sum_{i \in \mathcal{I}}\left(\left\langle D_{c, d} a_{i}, b_{i}\right\rangle+\left\langle a_{i}, D_{c, d} b_{i}\right\rangle\right) \\
& =\sum_{i \in \mathscr{I}} D_{a_{i}, b_{i}}(\langle c, d\rangle)=0,
\end{aligned}
$$

so that $D_{c, d}\left(H C_{1}(S)\right)=(0)$.

We now specialize to the case where our alternative algebra $S$ is the alternative torus $\mathfrak{A}$. Since $\mathfrak{A}$ is $\mathbb{Z}^{\nu}$-graded then so is $\mathfrak{A} \otimes_{k} \mathfrak{A}$, where if $x, y \in \mathfrak{A}$ are homogeneous then $\operatorname{deg}(x \otimes y)=\operatorname{deg} x+\operatorname{deg} y$. Clearly the $k$-subspace $I$ spanned by the elements in $(1.45)$ is homogeneous so that $\langle\mathfrak{A}, \mathfrak{A}\rangle$ inherits this $\mathbb{Z}^{\nu}$ grading as does $H C_{1}(\mathfrak{A})$. Indeed, $H C_{1}(S)$ is the kernel of the homogeneous map $\rho:\langle a, b\rangle \mapsto D_{a, b}$ of $\langle\mathfrak{A}, \mathfrak{A}\rangle$ onto $\operatorname{Inn}(\mathfrak{A})=\operatorname{Der}_{K}(\mathfrak{A})$ and by Lemma 1.46 this is a surjective Lie algebra homomorphism.

Recall that $\operatorname{dim} \mathfrak{A}_{\mathbf{a}}=1$ for $\mathbf{a} \in \mathbb{Z}^{\nu}$ so that if $\mathbf{a}, \mathbf{b} \in \mathbb{Z}^{\nu}$, then $\left\langle g^{\mathbf{b}}\left(g^{\mathbf{a}}\right)^{-1}, g^{\mathbf{a}}\right\rangle$ $=\left\langle g^{\mathbf{b}}\left(\alpha g^{\mathbf{a}}\right)^{-1}, \alpha g^{\mathbf{a}}\right\rangle$ for any non-zero $\alpha \in k$, so that for any $\mathbf{c} \in \mathbb{Z}^{\nu}$ we have

$$
\left\langle g^{\mathbf{b}}\left(g^{\mathbf{a}} g^{\mathbf{c}}\right)^{-1}, g^{\mathbf{a}} g^{\mathbf{c}}\right\rangle=\left\langle g^{\mathbf{b}}\left(g^{\mathbf{a}+\mathbf{c}}\right)^{-1}, g^{\mathbf{a}+\mathbf{c}}\right\rangle .
$$

Moreover, we will use the fact that if $\mathbf{b} \in \Gamma$, then $g^{\mathbf{b}} \in K$, the center of $\mathfrak{A}$, so that $\left\langle g^{\mathbf{b}}\left(g^{\mathbf{a}}\right)^{-1}, g^{\mathbf{a}}\right\rangle=\left\langle\left(g^{\mathbf{a}}\right)^{-1} g^{\mathbf{b}}, g^{\mathbf{a}}\right\rangle$.

In any octonion algebra $\mathbb{O}$, the inverse of an invertible element $x \in \mathbb{O}$ lies in the unital subalgebra generated by $x$ as $x^{-1}=n(x)^{-1} \bar{x}=n(x)^{-1}(2 t(x) 1-x)$. Since in an alternative algebra the subalgebra generated by two elements is associative, we have for invertible $x, y \in \mathbb{O}$ and arbitrary $a \in \mathbb{O}$ :

$$
(x y)^{-1}=y^{-1} x^{-1}, \quad\left(a x^{-1}\right) x=a=x\left(x^{-1} a\right)
$$

We will use these remarks freely in the computations which follow. Also, recall the definition of the elements $g_{i}$ before (1.33).

Definition 1.49. For $\mathbf{a} \in \Gamma$ and $\beta=\left(\beta_{1}, \ldots, \beta_{\nu}\right) \in k^{\nu}$ we let $\omega_{\mathbf{a}, \beta}=$ $\sum_{i=1}^{\nu} \beta_{i}\left\langle g^{\mathbf{a}} g_{i}^{-1}, g_{i}\right\rangle$. Sometimes we write $g^{\mathbf{a}} \omega_{\beta}$ for the element $\omega_{\mathbf{a}, \beta}$.

Lemma 1.50. (1) For $\mathbf{a} \in \Gamma, \beta \in \mathbb{Z}^{\nu}$ we have

$$
g^{\mathbf{a}} \omega_{\beta}=\omega_{\mathbf{a}, \beta}=\sum_{i=1}^{\nu} \beta_{i}\left\langle g^{\mathbf{a}} g_{i}^{-1}, g_{i}\right\rangle=\left\langle g^{\mathbf{a}}\left(g^{\beta}\right)^{-1}, g^{\beta}\right\rangle,
$$

where $\beta=\left(\beta_{1}, \ldots, \beta_{\nu}\right)$.

(2) For $\mathbf{a}, \mathbf{b} \in \Gamma, \alpha, \beta \in k^{\nu}$ we have

$$
\left(g^{\mathbf{a}} \Gamma_{\alpha}\right)\left(g^{\mathbf{b}} \omega_{\beta}\right)=g^{\mathbf{a}+\mathbf{b}} \omega_{(\alpha \mid \mathbf{b}) \beta+(\alpha \mid \beta) \mathbf{a}} .
$$

Proof. For $\mathbf{b}=\left(b_{1}, \ldots, b_{\nu}\right) \in \mathbb{Z}^{\nu}$ we let $|\mathbf{b}|=\sum_{i=1}^{\nu}\left|b_{i}\right|$ and use induction on $|\mathbf{b}|$. Clearly when $|\mathbf{b}|=0$ the result in (1) is true, and when $|\mathbf{b}|=1$ it also clearly holds, since we have $\left\langle g^{\mathbf{a}} g_{i}, g_{i}^{-1}\right\rangle=-\left\langle 1, g^{\mathbf{a}}\right\rangle-\left\langle g_{i}^{-1} g^{\mathbf{a}}, g_{i}\right\rangle=$ $-\left\langle g^{\mathbf{a}} g_{i}^{-1}, g_{i}\right\rangle$ because $\langle 1, x\rangle=0$ and $g^{\mathbf{a}} \in K=\mathscr{Z}(\mathfrak{A})$. Now let $\mathbf{b}^{\prime}=\mathbf{b}+\epsilon_{j} \in$ $\mathbb{Z}^{\nu}$ and suppose, by induction, the formula holds up to $|\mathbf{b}|$. We have

$$
\begin{aligned}
& \left\langle g^{\mathbf{a}}\left(g^{\mathbf{b}^{\prime}}\right)^{-1}, g^{\mathbf{b}^{\prime}}\right\rangle=\left\langle g^{\mathbf{a}}\left(g_{j}^{-1}\left(g^{\mathbf{b}}\right)^{-1}\right), g^{\mathbf{b}} g_{j}\right\rangle \\
& \quad=\left\langle g_{j}\left(g^{\mathbf{a}}\left(g_{j}^{-1}\left(g^{\mathbf{b}}\right)^{-1}\right)\right), g^{\mathbf{b}}\right\rangle+\left\langle\left(g^{\mathbf{a}}\left(g_{j}^{-1}\left(g^{\mathbf{b}}\right)^{-1}\right)\right) g^{\mathbf{b}}, g_{j}\right\rangle,
\end{aligned}
$$


by (1.48). Since $\mathbf{a} \in \Gamma$ this is

$$
\left\langle g^{\mathbf{a}}\left(g_{j}\left(g_{j}^{-1}\left(g^{\mathbf{b}}\right)^{-1}\right)\right), g^{\mathbf{b}}\right\rangle+\left\langle g^{\mathbf{a}}\left(g_{j}^{-1}\left(g^{\mathbf{b}}\right)^{-1}\right) g^{\mathbf{b}}, g_{j}\right\rangle,
$$

which, by our previous remarks, is just $\left\langle g^{\mathbf{a}}\left(g^{\mathbf{b}}\right)^{-1}, g^{\mathbf{b}}\right\rangle+\left\langle g^{\mathbf{a}} g_{j}^{-1}, g_{j}\right\rangle$. By induction this is $\sum_{i=1}^{\nu}\left(b_{i}+\delta_{i j}\right)\left\langle g^{\mathbf{a}} g_{i}^{-1}, g_{i}\right\rangle$ as desired. The case where $\mathbf{b}^{\prime}=\mathbf{b}-\epsilon_{j}$ is dealt with in a similar way. This proves (1).

As for (2) we let $\mathbf{a}, \mathbf{b} \in \Gamma, \alpha, \beta \in k^{\nu}$ and consider $\left(g^{\mathbf{a}} \Gamma_{\alpha}\right)\left(g^{\mathbf{b}} \omega_{\beta}\right)$. Using (1.47) and the fact that $g^{\mathbf{a}}, g^{\mathrm{b}}$ are central in $\mathfrak{A}$ we have

$$
\begin{aligned}
\left(g^{\mathbf{a}} \Gamma_{\alpha}\right)\left(g^{\mathbf{b}} \omega_{\beta}\right)= & \sum_{j=1}^{\nu} \beta_{j}(\alpha \mid \mathbf{b})\left\langle g^{\mathbf{a}} g^{\mathbf{b}} g_{j}^{-1}, g_{j}\right\rangle \\
& +\sum_{j=1}^{\nu} \beta_{j}\left(\alpha \mid-\epsilon_{j}\right)\left\langle g^{\mathbf{a}} g^{\mathbf{b}} g_{j}^{-1}, g_{j}\right\rangle \\
& +\sum_{j=1}^{\nu} \beta_{j}\left(\alpha \mid \epsilon_{j}\right)\left\langle g^{\mathbf{b}} g_{j}^{-1}, g^{\mathbf{a}} g_{j}\right\rangle
\end{aligned}
$$

Writing

$$
\left\langle g^{\mathbf{b}} g_{j}^{-1}, g^{\mathbf{a}} g_{j}\right\rangle=\left\langle g^{\mathbf{b}} g_{j}^{-1} g^{\mathbf{a}}, g_{j}\right\rangle+\left\langle g_{j} g^{\mathbf{b}} g_{j}^{-1}, g^{\mathbf{a}}\right\rangle
$$

and cancelling yields

$$
\sum_{j=1}^{\nu} \beta_{j}(\alpha \mid \mathbf{b})\left\langle g^{\mathbf{a}+\mathbf{b}} g_{j}^{-1}, g_{j}\right\rangle+\sum_{i=1}^{\nu} \beta_{j}\left(\alpha \mid \epsilon_{j}\right)\left\langle g^{\mathbf{b}}, g^{\mathbf{a}}\right\rangle .
$$

But $\left\langle g^{\mathbf{b}}, g^{\mathbf{a}}\right\rangle=\left\langle g^{\mathbf{b}+\mathbf{a}}\left(g^{\mathbf{a}}\right)^{-1}, g^{\mathbf{a}}\right\rangle$ as $\mathbf{a}, \mathbf{b} \in \Gamma$ so that

$$
\left(g^{\mathbf{a}} \Gamma_{\alpha}\right)\left(g^{\mathbf{b}} \omega_{\beta}\right)=(\alpha \mid \mathbf{b}) g^{\mathbf{a}+\mathbf{b}} \omega_{\beta}+(\alpha \mid \beta) g^{\mathbf{a}+\mathbf{b}} \omega_{\alpha}=g^{\mathbf{a}+\mathbf{b}} \omega_{(\alpha \mid \mathbf{b}) \beta+(\alpha \mid \beta) \mathbf{a}},
$$

as desired.

Definition 1.51. Define a $k$-linear map $\varepsilon: \mathfrak{A} \rightarrow k$ by saying that

$$
\varepsilon\left(g^{\mathbf{a}}\right)= \begin{cases}1 & \text { if } \mathbf{a}=0, \\ 0 & \text { if } \mathbf{a} \in \mathbb{Z}^{\nu}, \mathbf{a} \neq 0 .\end{cases}
$$

By Remark $1.22, \varepsilon$ vanishes on $[\mathfrak{A}, \mathfrak{A}]=(\mathfrak{A}, \mathfrak{A}, \mathfrak{A})$. Defining a $k$-bilinear form $(\cdot, \cdot): \mathfrak{A} \times \mathfrak{A} \rightarrow k$ by

$$
(x, y)=\varepsilon(x y) \text { for } x, y \in \mathfrak{A},
$$

we find that $(\cdot, \cdot)$ is symmetric and associative in the sense that

$$
(x y, z)=(x, y z) \text { for all } x, y, z \in \mathfrak{A} \text {. }
$$

Moreover, it is clear that this form is nondegenerate. By the definition of $\varepsilon$ we find that

$$
\varepsilon\left(d_{i}(x)\right)=0 \text { for any } x \in \mathfrak{A} \text {, and } 1 \leq i \leq \nu .
$$

From this we have $\varepsilon\left(d_{i}(x y)\right)=0$ for all $x, y \in \mathfrak{A}$ so that

$$
\left(d_{i}(x), y\right)=-\left(x, d_{i}(y)\right) \text { for } 1 \leq i \leq \nu, x, y \in \mathfrak{A} .
$$

That is, each degree derivation $d_{i}$ is skew-symmetric relative to the form $(\cdot, \cdot)$. We will use this in our next result. Also, recall that $\langle\mathfrak{A}, \mathfrak{A}\rangle$ and $H C_{1}(\mathfrak{A})$ are both $\mathbb{Z}^{\nu}$-graded with $\operatorname{deg}\left\langle g^{\mathbf{a}}, g^{\mathbf{b}}\right\rangle=\mathbf{a}+\mathbf{b}$ for $\mathbf{a}, \mathbf{b} \in \mathbb{Z}^{\nu}$. 
Lemma 1.56. For our alternative torus $\mathfrak{A}$ we have that $\langle\mathfrak{A}, \mathfrak{A}\rangle_{\mathbf{a}}$ for any $\mathbf{a} \in \mathbb{Z}^{\nu}$ is spanned by the elements $\left\langle g^{\mathbf{a}} g_{i}^{-1}, g_{i}\right\rangle$. We also have $\operatorname{dim}_{k} H C_{1}(\mathfrak{A})_{0}=\nu$.

Proof. Since $\left\langle g^{\mathbf{a}}, g^{\mathbf{b}}\right\rangle=\left\langle g^{\mathbf{a}}\left(g^{\mathbf{b}}\left(g^{\mathbf{b}}\right)^{-1}\right), g^{\mathbf{b}}\right\rangle$ is a multiple of $\left\langle g^{\mathbf{a}+\mathbf{b}}\left(g^{\mathbf{b}}\right)^{-1}, g^{\mathbf{b}}\right\rangle$ and of degree $\mathbf{a}+\mathbf{b}$ we see that $\langle\mathfrak{A}, \mathfrak{A}\rangle_{\mathbf{a}}$ is spanned by elements $\left\langle g^{\mathbf{a}}\left(g^{\mathbf{b}}\right)^{-1}, g^{\mathbf{b}}\right\rangle$ for $\mathbf{b} \in \mathbb{Z}^{\nu}$. Moreover, $\left\langle g^{\mathbf{a}} g_{i}^{-1}, g_{i}\right\rangle$ is in $\langle\mathfrak{A}, \mathfrak{A}\rangle_{\mathbf{a}}$ for $1 \leq i \leq \nu$. We show that if $\mathbf{b}=\sum_{i=1}^{\nu} b_{i} \epsilon_{i} \in \mathbb{Z}^{\nu}$ then $\left\langle g^{\mathbf{a}}\left(g^{\mathbf{b}}\right)^{-1}, g^{\mathbf{b}}\right\rangle$ is a linear combination of the elements $\left\langle g^{\mathbf{a}} g_{i}^{-1}, g_{i}\right\rangle$ by using induction on $|\mathbf{b}|=\sum_{i=1}^{\nu}\left|b_{i}\right|$. This is clear if $|\mathbf{b}|=0$ or if $|\mathbf{b}|=1$. Moreover as

$$
\left\langle g^{\mathbf{a}}\left(g^{\mathbf{b}}\right)^{-1}, g^{\mathbf{b}}\right\rangle=-\left\langle\left(g^{\mathbf{b}}\right)^{-1} g^{\mathbf{b}}, g^{\mathbf{a}}\right\rangle-\left\langle g^{\mathbf{b}} g^{\mathbf{a}},\left(g^{\mathbf{b}}\right)^{-1}\right\rangle=-\left\langle g^{\mathbf{b}} g^{\mathbf{a}},\left(g^{\mathbf{b}}\right)^{-1}\right\rangle
$$

and this is clearly a multiple of $\left\langle g^{\mathbf{a}} g^{\mathbf{b}},\left(g^{\mathbf{b}}\right)^{-1}\right\rangle$ then we can suppose (replacing $\mathbf{b}$ and $\mathbf{b}^{\prime}$ if necessary ) that $\mathbf{b}=\mathbf{b}^{\prime}+\epsilon_{i}$ for some $\mathbf{b}^{\prime} \in \mathbb{Z}^{\nu}$ with $\left|\mathbf{b}^{\prime}\right|<|\mathbf{b}|$. Then $g^{\mathbf{b}}$ is a multiple of $g^{\mathbf{b}^{\prime}} g_{i}$ so that

$$
\left\langle g^{\mathbf{a}}\left(g^{\mathbf{b}}\right)^{-1}, g^{\mathbf{b}}\right\rangle=\left\langle g^{\mathbf{a}}\left(g_{i}^{-1}\left(g^{\mathbf{b}^{\prime}}\right)^{-1}\right), g^{\mathbf{b}^{\prime}} g_{i}\right\rangle
$$

and this is a multiple of $\left\langle\left(g^{\mathbf{a}} g_{i}^{-1}\right)\left(g^{\mathbf{b}^{\prime}}\right)^{-1}, g^{\mathbf{b}^{\prime}} g_{i}\right\rangle$. Using (1.43) and (1.44) we see this is a linear combination of $\left\langle\left(g^{\mathbf{b}^{\prime}}\right)^{-1}\left(g^{\mathbf{b}^{\prime}} g_{i}\right), g^{\mathbf{a}^{\prime}} g_{i}^{-1}\right\rangle$ and $\left\langle\left(g^{\mathbf{b}^{\prime}} g_{i}\right)\left(g^{\mathbf{a}} g_{i}^{-1}\right)\right.$, $\left.\left(g^{\mathbf{b}^{\prime}}\right)^{-1}\right\rangle$. Clearly the first of these is in the $k$-span of $\left\langle g^{\mathbf{a}} g_{i}^{-1}, g_{i}\right\rangle$ while the second term is a multiple of $\left\langle g^{\mathbf{a}} g^{\mathbf{b}^{\prime}},\left(g^{\mathbf{b}^{\prime}}\right)^{-1}\right\rangle$, and so since $\left|\mathbf{b}^{\prime}\right|<|\mathbf{b}|$ we can finish by induction. This proves that $\langle\mathfrak{A}, \mathfrak{A}\rangle_{\mathbf{a}}$ is spanned by $\left\langle g^{\mathbf{a}} g_{i}^{-1}, g_{i}\right\rangle$ for $1 \leq i \leq \nu$.

For the second part of the lemma we define linear functionals $\chi_{i}: \mathfrak{A} \otimes \mathfrak{A} \rightarrow k$ for $1 \leq i \leq \nu$ by

$$
\chi_{i}(x \otimes y)=\left(d_{i}(x), y\right) \text { for all } x, y \in \mathfrak{A} .
$$

As each $d_{i}$ is skew-symmetric relative to $(\cdot, \cdot)$ (see 1.55) then $\chi_{i}(x \otimes y+y \otimes$ $x)=0$ for $x, y \in \mathfrak{A}$. Moreover, using that the form $(\cdot, \cdot)$ is symmetric and associative we have for $x, y, z \in \mathfrak{A}$ that

$$
\begin{aligned}
\chi_{i}(x y & \otimes z+y z \otimes x+z x \otimes y) \\
& =\left(d_{i}(x y), z\right)+\left(d_{i}(y z), x\right)+\left(d_{i}(z x), y\right) \\
& =\left(d_{i}(x) y, z\right)+\left(x d_{i}(y), z\right)+\left(x, d_{i}(y z)\right)+\left(y, d_{i}(z x)\right) \\
& =\left(d_{i}(x), y z\right)+\left(d_{i}(y), z x\right)+\left(x, d_{i}(y z)\right)+\left(y, d_{i}(z x)\right) \\
& =0 .
\end{aligned}
$$

Thus, $\chi_{i}$ induces a functional, which we also denote by $\chi_{i}$, on $\langle\mathfrak{A}, \mathfrak{A}\rangle$ satisfying

$$
\chi_{i}(\langle x, y\rangle)=\left(d_{i}(x), y\right) \text { for all } x, y \in \mathfrak{A} .
$$

We know that the elements $\left\langle g_{i}^{-1}, g_{i}\right\rangle, 1 \leq i \leq \nu$ span the subspace $\langle\mathfrak{A}, \mathfrak{A}\rangle_{0}=$ $H C_{1}(\mathfrak{A})_{0}$ and if $\sum_{i=1}^{\nu} \alpha_{i}\left\langle g_{i}^{-1}, g_{i}\right\rangle=0$ we apply the functional $\chi_{j}$ to this element and obtain

$$
0=\sum_{i=1}^{\nu} \alpha_{i}\left(d_{j}\left(g_{i}^{-1}\right), g_{i}\right)
$$

This gives

$$
0=-\sum_{i=1}^{\nu} \alpha_{i} \delta_{i j}\left(g_{i}^{-1}, g_{i}\right)=-\alpha_{j}
$$

and so $\left\langle g_{1}^{-1}, g_{1}\right\rangle, \ldots,\left\langle g_{\nu}^{-1}, g_{\nu}\right\rangle$ are linearly independent, as desired. 
In our next result we compute $\operatorname{dim}_{k} H C_{1}(\mathfrak{A})_{\mathbf{a}}$ for $\mathbf{a} \in \Gamma$. The result is the same as in the case when the coordinates are the quantum torus $\mathbb{C}_{\mathbf{q}}$ and $\Gamma$ is the radical of $f_{\mathbf{q}}$, see [BGK].

Lemma 1.57. (a) For $\mathbf{a} \in \Gamma, \alpha \in k^{\nu}$ the derivation $\Gamma_{\mathbf{a}, \alpha}$ is skew-symmetric relative to the form $(\cdot, \cdot)$ on $\mathfrak{A}$ if and only if $(\alpha \mid \mathbf{a})=0$.

(b) For $\mathbf{a} \in \Gamma$ we have $\langle\mathfrak{A}, \mathfrak{A}\rangle_{\mathfrak{a}}=H C_{1}(\mathfrak{A})_{\mathbf{a}}$.

(c) $\operatorname{dim}_{k} H C_{1}(\mathfrak{A})_{\mathbf{a}}=\nu-1$ if $\mathbf{a} \in \Gamma \backslash\{0\}$.

Proof. Note that $\Gamma_{\mathbf{a}, \alpha}$ is skew-symmetric relative to the form $(\cdot, \cdot)$ iff $\varepsilon\left(\Gamma_{\mathbf{a}, \alpha}(x y)\right)=0$ for all $x, y \in \mathfrak{A}$. This is equivalent to $\varepsilon\left(\Gamma_{\mathbf{a}, \alpha}\left(g^{\mathbf{b}}\right)\right)=0$ for all $\mathbf{b} \in \mathbb{Z}^{\nu}$, so $\Gamma_{\mathbf{a}, \alpha}$ is skew-symmetric $\Leftrightarrow(\alpha \mid \mathbf{b}) \varepsilon\left(g^{\mathbf{a}+\mathbf{b}}\right)=0$ for all $\mathbf{b} \in$ $\mathbb{Z}^{\nu} \Leftrightarrow(\alpha \mid \mathbf{a})=0$. This proves $(\mathbf{a})$.

For (b) we note that we have the Lie algebra homomorphism $\rho:\langle\mathfrak{A}, \mathfrak{A}\rangle \rightarrow$ $\operatorname{Der}_{K}(\mathfrak{A})$ given by $\rho(\langle x, y\rangle)=D_{x, y}$ for all $x, y \in \mathfrak{A}$ (see Lemma 1.46). This is homogeneous in the $\mathbb{Z}^{\nu}$ grading. Now by Theorem 1.40 we have $\operatorname{Der}_{K}(\mathfrak{A})=$ $\bigoplus_{\mathbf{a} \in \mathbb{Z}^{\nu} \backslash \Gamma} \mathscr{D}_{\mathbf{a}}$ so that if $\mathbf{a} \in \Gamma$ then $\langle\mathfrak{A}, \mathfrak{A}\rangle_{\mathbf{a}} \subseteq \operatorname{ker} \rho=H C_{1}(\mathfrak{A})$ and hence $\langle\mathfrak{A}, \mathfrak{A}\rangle_{\mathfrak{a}}=H C_{1}(\mathfrak{A})_{\mathbf{a}}$ as desired.

To prove (c) note that for $\mathbf{a} \in \Gamma \backslash\{0\},\langle\mathfrak{A}, \mathfrak{A}\rangle_{\mathbf{a}}=\left\{g^{\mathbf{a}} \omega_{\beta} \mid \beta \in k^{\nu}\right\}$ by Lemma $1.50(1)$ and $\langle\mathfrak{A}, \mathfrak{A}\rangle_{\mathfrak{a}}$ is spanned by $\left\langle g^{\mathfrak{a}} g_{i}^{-1}, g_{i}\right\rangle, 1 \leq i \leq \nu$. If $g^{\mathfrak{a}} \omega_{\beta}=0$, then $\left(g^{\mathbf{a}}\right)^{-1} \Gamma_{\alpha}\left(g^{\mathbf{a}} \omega_{\beta}\right)=0$ and this is a non-zero multiple of $\omega_{(\alpha \mid \mathbf{a}) \beta+(\alpha \mid \beta)(-\mathbf{a})}$ by Lemma $1.50(2)$. If $(\alpha \mid \mathbf{a})=0$, then we get $\omega_{(\alpha \mid \beta)(-\mathbf{a})}=0$ so since we know $\left\langle g_{i}^{-1}, g_{i}\right\rangle$ for $1 \leq i \leq \nu$ are linearly independent, we arrive at $(\alpha \mid \beta)=0$. Thus, for any $\alpha \in k^{\nu}$ satisfying $(\mathbf{a} \mid \alpha)=0$ we have $(\alpha \mid \beta)=0$, so this forces $\beta \in k \mathbf{a}$. On the other hand, if $\beta \in k \mathbf{a}$, then $g^{\mathbf{a}} \omega_{\beta}=0$ because $g^{\mathbf{a}} \omega_{\mathbf{a}}=$ $\left\langle g^{\mathbf{a}}\left(g^{\mathbf{a}}\right)^{-1}, g^{\mathbf{a}}\right\rangle=\left\langle 1, g^{\mathbf{a}}\right\rangle=0$ by Lemma 1.50(1). It follows that $\operatorname{dim}_{k}\langle\mathfrak{A}, \mathfrak{A}\rangle_{\mathbf{a}}=$ $\operatorname{dim}_{k} H C_{1}(\mathfrak{A})_{\mathbf{a}}=\nu-1$.

In our next result we determine $\langle\mathfrak{A}, \mathfrak{A}\rangle_{\mathfrak{a}}$ and $H C_{1}(\mathfrak{A})_{\mathfrak{a}}$ when $\mathbf{a} \in \mathbb{Z}^{\nu} \backslash \Gamma$.

Lemma 1.58. Let $\mathbf{a} \in \mathbb{Z}^{\nu} \backslash \Gamma$, then $H C_{1}(\mathfrak{A})_{\mathbf{a}}=(0)$ and $\operatorname{dim}_{k}\langle\mathfrak{A}, \mathfrak{A}\rangle_{\mathfrak{a}}=2$.

Proof. Recall that $\mathfrak{A}$ is a free $K$-module of rank 8 with basis $u_{1}=1, u_{2}, \ldots$, $u_{8}$ and that $g_{1}=u_{2}, g_{2}=u_{3}, g_{3}=u_{5}$ generate $\mathfrak{A}$ as a $K$-algebra. Thus, any monomial in $\mathfrak{A}$ can be written as $g^{\mathbf{a}} u_{i}$ for some $\mathbf{a} \in \Gamma, 1 \leq i \leq 8$. Then, by (1.44) we have

$$
\left\langle\left(g^{\mathbf{a}} u_{i}\right)\left(g^{\mathbf{b}} u_{j}\right), g^{\mathbf{c}}\right\rangle+\left\langle\left(g^{\mathbf{b}} u_{j}\right) g^{\mathbf{c}}, g^{\mathbf{a}} u_{i}\right\rangle+\left\langle g^{\mathbf{c}}\left(g^{\mathbf{a}} u_{i}\right), g^{\mathbf{b}} u_{j}\right\rangle=0,
$$

for $\mathbf{a}, \mathbf{b}, \mathbf{c} \in \Gamma, 1 \leq i, j \leq 8$. This gives

$$
\left\langle g^{\mathbf{a}+\mathbf{b}} u_{i} u_{j}, g^{\mathbf{c}}\right\rangle+\left\langle g^{\mathbf{b}+\mathbf{c}} u_{j}, g^{\mathbf{a}} u_{i}\right\rangle+\left\langle g^{\mathbf{a}+\mathbf{c}} u_{i}, g^{\mathbf{b}} u_{j}\right\rangle=0 .
$$

Interchanging $g^{\mathbf{a}} u_{i}$ and $g^{\mathbf{b}} u_{j}$ gives us

$$
\left\langle g^{\mathbf{a}+\mathbf{b}} u_{j} u_{i}, g^{\mathbf{c}}\right\rangle+\left\langle g^{\mathbf{a}+\mathbf{c}} u_{i}, g^{\mathbf{b}} u_{j}\right\rangle+\left\langle g^{\mathbf{b}+\mathbf{c}} u_{j}, g^{\mathbf{a}} u_{i}\right\rangle=0 .
$$

Adding (1.59) and (1.60) and dividing by 2 yield

$$
\left\langle g^{\mathbf{a}+\mathbf{b}}\left(\frac{u_{i} u_{j}+u_{j} u_{i}}{2}\right), g^{\mathbf{c}}\right\rangle+\left\langle g^{\mathbf{b}+\mathbf{c}} u_{j}, g^{\mathbf{a}} u_{i}\right\rangle+\left\langle g^{\mathbf{a}+\mathbf{c}} u_{i}, g^{\mathbf{b}} u_{j}\right\rangle=0 .
$$

Now if $2 \leq i \neq j \leq 8$ then $u_{i} u_{j}+u_{j} u_{i}=0$, so we get

$$
\left\langle g^{\mathbf{b}+\mathbf{c}} u_{j}, g^{\mathbf{a}} u_{i}\right\rangle+\left\langle g^{\mathbf{a}+\mathbf{c}} u_{i}, g^{\mathbf{b}} u_{j}\right\rangle=0 .
$$


Taking $b=0$ in (1.61) gives us, by using (1.43),

$$
\left\langle u_{j}, g^{\mathbf{a}+\mathbf{c}} u_{i}\right\rangle=\left\langle g^{\mathbf{c}} u_{j}, g^{\mathbf{a}} u_{i}\right\rangle,
$$

for $\mathbf{a}, \mathbf{c}, \in \Gamma$ and $2 \leq i \neq j \leq 8$.

Taking the difference of (1.59) and (1.60) gives us $\left\langle g^{\mathbf{a}+\mathbf{b}}\left(u_{i} u_{j}-u_{j} u_{i}\right), g^{\mathbf{c}}\right\rangle=0$. Since $i$ and $j$ can be chosen so that $u_{i} u_{j}-u_{j} u_{i}$ is a non-zero multiple of $u_{m}$ for any $m$ satisfying $2 \leq m \leq 8$, we obtain

$$
\left\langle g^{\mathbf{a}} u_{m}, g^{\mathbf{b}}\right\rangle=0,
$$

for any $\mathbf{a}, \mathbf{b} \in \Gamma$ and $2 \leq m \leq 8$. Thus, (1.62) holds in the case when exactly one of the two distinct indices $i$ and $j$ equals 1 .

Now fix $\mathbf{a} \in \mathbb{Z}^{\nu} \backslash \Gamma$. By Lemma 1.56 we know that the elements $\left\langle g^{\mathrm{a}} g_{i}^{-1}, g_{i}\right\rangle$ for $1 \leq i \leq 8$ span $\langle\mathfrak{A}, \mathfrak{A}\rangle_{\mathfrak{a}}$. As $g_{i} \in K$ for $4 \leq i \leq \nu$ we find that the 3 elements $\left\langle g^{\mathbf{a}} g_{i}^{-1}, g_{i}\right\rangle$ for $i=1,2,3$ span $\langle\mathfrak{A}, \mathfrak{A}\rangle_{\mathbf{a}}$. We show that these 3 elements are linearly dependent. Since $\mathbf{a} \notin \Gamma$ we can write $g^{\mathbf{a}}=g^{\mathbf{b}} u_{j}$ for some $\mathbf{b} \in \Gamma, 2 \leq j \leq 8$. If $j \in\{2,3,5\}$, then the element with $g_{i}=u_{j}$ above satisfies

$$
\left\langle g^{\mathbf{a}} g_{i}^{-1}, g_{i}\right\rangle=\left\langle g^{\mathbf{b}} u_{j} u_{j}^{-1}, u_{j}\right\rangle=\left\langle g^{\mathbf{b}}, u_{j}\right\rangle=0,
$$

showing our 3 elements span at most a 2 -dimensional space. If $j \in\{4,6,7\}$, then say for convenience that $j=4$, so from [S], p. 5 we find that $u_{j}=u_{4}=$ $u_{2} u_{3}=g_{1} g_{2}$ so that

$$
\left\langle g^{\mathbf{a}} g_{1}^{-1}, g_{1}\right\rangle=\left\langle g^{\mathbf{b}} u_{4} u_{2}^{-1}, u_{2}\right\rangle=\left\langle g^{\mathbf{b}}\left(u_{2} u_{3}\right) u_{2}^{-1}, u_{2}\right\rangle
$$

is a multiple of $\left\langle g^{\mathbf{b}} u_{3}, u_{2}\right\rangle$ and so is

$$
\left\langle g^{\mathbf{a}} g_{2}^{-1}, g_{2}\right\rangle=\left\langle g^{\mathbf{b}} u_{4} u_{3}^{-1}, u_{3}\right\rangle=\left\langle g^{\mathbf{b}}\left(u_{2} u_{3}\right) u_{3}^{-1}, u_{3}\right\rangle
$$

by (1.62). Again we can conclude that elements span a space which is at most 2-dimensional. As $u_{2} u_{5}=-u_{6}, u_{3} u_{5}=-u_{7}$ the same argument for $j=4$ works for $j=6$ and $j=7$, so we only consider $j=8$ and $g^{\mathbf{a}}=g^{\mathbf{b}} u_{8}$. Now $\left(u_{2} u_{3}\right) u_{5}=u_{8}$, so we find $\left\langle g^{\mathbf{a}} g_{1}^{-1}, g_{1}\right\rangle=\left\langle g^{\mathbf{b}}\left(\left(u_{2} u_{3}\right) u_{5}\right) u_{2}^{-1}, u_{2}\right\rangle$ is a multiple of $\left\langle g^{\mathbf{b}} u_{3} u_{5}, u_{2}\right\rangle$, which is a linear combination of the two elements $\left\langle g^{\mathbf{b}} u_{5} u_{2}, u_{3}\right\rangle$ and $\left\langle g^{\mathbf{b}} u_{2} u_{3}, u_{5}\right\rangle$ by (1.44). The first of these is a non-zero multiple of $\left\langle g^{\mathbf{a}} g_{2}^{-1}, g_{2}\right\rangle$ and the second is a non-zero multiple of $\left\langle g^{\mathbf{a}} g_{3}^{-1}, g_{3}\right\rangle$ so again we have a space of at most 2 dimensions. Thus. for $\mathbf{a} \in \mathbb{Z}^{\nu} \backslash \Gamma$, we have $\operatorname{dim}\langle\mathfrak{A}, \mathfrak{A}\rangle_{\mathbf{a}} \leq 2$.

To finish the proof, recall that we have a Lie algebra epimorphism $\rho$ : $\langle\mathfrak{A}, \mathfrak{A}\rangle \rightarrow \operatorname{Der}_{K}(\mathfrak{A})$ which is homogeneous and has $H C_{1}(\mathfrak{A})=\operatorname{ker} \rho$. We know that $\operatorname{Der}_{K}(\mathfrak{A})=\bigoplus_{\mathbf{a} \in \mathbb{Z}^{\nu} \backslash \Gamma} \mathscr{D}_{\mathbf{a}}$ and that $\operatorname{dim}_{k} \mathscr{D}_{\mathbf{a}}=2$ for any $\mathbf{a} \in \mathbb{Z}^{\nu} \backslash \Gamma$ so it follows that for such elements a we have $\rho$ is injective on $\langle\mathfrak{A}, \mathfrak{A}\rangle_{\mathbf{a}}$ and $H C_{1}(\mathfrak{A})_{\mathbf{a}}=(0)$. This is what we want.

For easy reference we state a result summarizing what we know about $\langle\mathfrak{A}, \mathfrak{A}\rangle$ and $H C_{1}(\mathfrak{A})$.

Theorem 1.64. Let $\mathfrak{A}$ be the alternative torus. Then

$$
\operatorname{dim}_{k} H C_{1}(\mathfrak{A})_{\mathbf{a}}= \begin{cases}\nu & \text { if } \mathbf{a}=0, \\ \nu-1 & \text { if } \mathbf{a} \in \Gamma \backslash\{0\}, \\ 0 & \text { if } \mathbf{a} \in \mathbb{Z}^{\nu} \backslash \Gamma .\end{cases}
$$


Moreover, for $\mathbf{a} \in \Gamma$ we have $H C_{1}(\mathfrak{A})_{\mathbf{a}}=\langle\mathfrak{A}, \mathfrak{A}\rangle_{\mathbf{a}}$ so

$$
H C_{1}(\mathfrak{A})=\bigoplus_{\mathbf{a} \in \Gamma} H C_{1}(\mathfrak{A})_{\mathbf{a}}=\bigoplus_{\mathbf{a} \in \Gamma}\langle\mathfrak{A}, \mathfrak{A}\rangle_{\mathbf{a}}
$$

while if $\mathbf{a} \in \mathbb{Z}^{\nu} \backslash \Gamma$ then $\operatorname{dim}_{k}\langle\mathfrak{A}, \mathfrak{A}\rangle_{\mathbf{a}}=2$.

\section{2. $A_{2}$-GRADED LIE ALGEBRAS WITH ALTERNATIVE COORDINATES}

The purpose of this section is to study Lie algebras with alternative coordinates which are $A_{2}$-graded in the sense of [BM]. We show they are central extension of a perfect Lie algebra, $\operatorname{psl}_{3}(S)$, where $S$ is an alternative algebra over the field $k$. As in Section 1 we continue to assume that the characteristic of $k$ is not 2 or 3 . We show the cyclic homology, $H C_{1}(S)$, determines the Schur multiplier, so is the kernel of the natural map of the universal covering algebra of $\operatorname{psl}_{3}(S)$ to this algebra. We then go on to study the properties of these algebras which we need for our classification of the quasi-simple Lie algebras. In particular, we deal with derivations and invariant symmetric bilinear forms and when economy is to be gained by working with the alternative torus $\mathfrak{A}$, rather than a general alternative $k$-algebra $S$, we do so. All of the material here is important when we come to the classification of the next section. The reader should consult $[\mathrm{F}]$ (also [AF]) and [Se] for information and other viewpoints on the Lie algebra we consider. One should note that both of these references deal with Lie algebras of type $A_{2}$ coordinatized by alternative algebras, but from differing viewpoints, which bring out different aspects of the structure. We have chosen to follow [Se] since it seems to us to conform to the structural features which one encounters when dealing with the covering quasi-simple Lie algebras.

We begin by recalling a simple lemma about the Lie algebra $s l_{3}(k)$ which we will need later. As usual, $\operatorname{Tr}(x)$ denotes the trace of the matrix (or linear map) $x$.

Lemma 2.1. For any $x, y, z \in s l_{3}(k)$ we have that

$$
\begin{aligned}
& x y z+x z y+y x z+y z x+z x y+z y x \\
& \quad-\operatorname{Tr}(x y) z-\operatorname{Tr}(y z) x-\operatorname{Tr}(z x) y-\operatorname{Tr}(x y z+y x z) I_{3}=0
\end{aligned}
$$

where $I_{3}$ is the $3 \times 3$ identity matrix.

Proof. It is an easy matter to check that

$$
x^{3}-\frac{1}{2} \operatorname{Tr}\left(x^{2}\right) x-\frac{1}{3} \operatorname{Tr}\left(x^{3}\right) I_{3}=0
$$

for any $x \in s l_{3}(k)$. Linearizing by replacing $x$ by $x+y$ in this and then canceling terms yield that

$$
\begin{aligned}
x^{2} y & +x y x+x y^{2}+y x^{2}+y x y+y^{2} x \\
& -\frac{1}{2} \operatorname{Tr}\left(x^{2}\right) y-\operatorname{Tr}(x y)(x+y)-\frac{1}{2} \operatorname{Tr}\left(y^{2}\right) x-\operatorname{Tr}\left(x^{2} y+x y^{2}\right) I_{3}=0
\end{aligned}
$$

for all $x, y \in s l_{3}(k)$. Finally, linearizing again by replacing $x$ by $x+z$ in this latter identity and then canceling terms give the desired identity. 
Recall from Lemma 1.46 that if $S$ is an alternative $k$-algebra and if $\mathscr{E}$ is a $k$-vector space such that there is a surjective map $\{\cdot, \cdot\}: S \otimes S \rightarrow \mathscr{E}$ satisfying $\{a, b\}+\{b, a\}=0$ and $\{a b, c\}+\{b c, a\}+\{c a, b\}=0$ for all $a, b, c \in S$ and a linear map $\pi: \mathscr{E} \rightarrow \operatorname{Inn}(S)$ satisfying $\pi(\{a, b\})=D_{a, b}$ for all $a, b \in S$, then the $k$-bilinear extension of $[\cdot, \cdot]$ defined by

$$
[\{a, b\},\{c, d\}]=\left\{D_{a, b} c, d\right\}+\left\{c, D_{a, b} d\right\}
$$

for all $a, b, c, d \in S$ to a map $[\cdot, \cdot]: \mathscr{E} \times \mathscr{E} \rightarrow \mathscr{E}$ makes $\mathscr{E}$ into a Lie algebra. In particular, we have two examples of this situation, one by taking $\mathscr{E}=\operatorname{Inn}(S)$ and $\{a, b\}=D_{a, b}, \pi(\{a, b\})=D_{a, b}$ and the other by taking $\mathscr{E}=\langle S, S\rangle$ and $\{a, b\}=\langle a, b\rangle$ and $\pi=\rho$ so $\rho(\langle a, b\rangle)=D_{a, b}$. Our next result shows how to build a Lie algebra of type $A_{2}$ coordinatized by $S$ from this data. These algebras will turn out to be the cores of our quasi-simple Lie algebras.

Proposition 2.2. Let $S$ be an alternative $k$-algebra with identity and $\mathscr{E}$ a $k$ vector space together with a surjective bilinear map $\{\cdot, \cdot\}: S \otimes S \rightarrow \mathscr{E}$ and a $k$-linear map $\pi: \mathscr{E} \rightarrow \operatorname{Inn}(S)$ satisfying

(i) $\{a, b\}+\{b, a\}=0$,

(ii) $\{a b, c\}+\{b c, a\}+\{c a, b\}=0$,

(iii) $\pi(\{a, b\})=D_{a, b}$, for all $a, b, c \in S$.

Let $\mathfrak{g}=\mathscr{E} \oplus\left(s l_{3}(k) \otimes S\right)$ and define a map $[\cdot, \cdot \cdot]: \mathfrak{g} \times \mathfrak{g} \rightarrow \mathfrak{g}$ by bilinear extension of the formulas

$$
\begin{aligned}
& {[x \otimes a, y \otimes b]=\frac{1}{3} \operatorname{Tr}(x y)\{a, b\}+[x, y] \otimes \frac{a b+b a}{2}} \\
& +\left(x y+y x-\frac{2}{3} \operatorname{Tr}(x y) I_{3}\right) \otimes \frac{a b-b a}{2}, \\
& {[\{a, b\}, x \otimes c]=-[x \otimes c,\{a, b\}]=x \otimes D_{a, b} c,} \\
& {[\{a, b\},\{c, d\}]=\left\{D_{a, b} c, d\right\}+\left\{c, D_{a, b} d\right\},}
\end{aligned}
$$

for $a, b, c, d \in S, x, y \in l_{3}(k)$. Then $\mathfrak{g}$ is a Lie algebra with the above bracket product $[\cdot, \cdot]$.

Remark 2.3. We write $(x, y)_{o}$ for the term $x y+y x-\frac{2}{3} \operatorname{Tr}(x y) I_{3}$ in the above when $x, y \in s l_{3}(k)$. Note that $(x, y)_{o} \in s l_{3}(k)$.

Proof. To prove this, we need only verify the Jacobi identity is satisfied in the following cases. We note that Lemma 1.46 already says that this product defines a Lie algebra structure on $\mathscr{E}$. We write

$$
J(A, B, C)=[[A, B], C]+[[B, C], A]+[[C, A], B] .
$$

Case 1. Assume $A=\{a, b\}, B=\{c, d\} \in \mathscr{E}$ and $C=x \otimes e \in s l_{3}(k) \otimes S$. Then we have that

$$
\begin{gathered}
J(A, B, C)=x \otimes\left[D_{a, b}, D_{c, d}\right] e-x \otimes D_{a, b} D_{c, d} e \\
+x \otimes D_{c, d} D_{a, b} e=0
\end{gathered}
$$


Case 2. Assume $A=\{a, b\} \in \mathscr{E}, B=x \otimes c, C=y \otimes d \in s l_{3}(k) \otimes S$. Then

$$
\begin{aligned}
J(A, B & C)=\left[x \otimes D_{a, b} c, y \otimes+d\right]+\left[\frac{1}{3} \operatorname{Tr}(x y)\{c, d\}+[x, y]\right. \\
& \left.\otimes \frac{c d+d c}{2}+(x, y)_{o} \otimes \frac{c d-d c}{2},\{a, b\}\right]+\left[x \otimes c, y \otimes D_{a, b} d\right] \\
= & \frac{1}{3} \operatorname{Tr}(x y)\left\{D_{a, b} c, d\right\}+[x, y] \otimes \frac{\left(D_{a, b} c\right) d+d D_{a, b} c}{2} \\
& +(x, y)_{o} \otimes \frac{\left(D_{a, b} c\right) d-d D_{a, b} c}{2} \\
& +\frac{1}{3} \operatorname{Tr}(x y)[\{c, d\},\{a, b\}]-[x, y] \otimes D_{a, b}\left(\frac{c d+d c}{2}\right) \\
& -(x, y)_{o} \otimes D_{a, b}\left(\frac{c d-d c}{2}\right) \\
& +\frac{1}{3} \operatorname{Tr}(x y)\left\{c, D_{a, b} d\right\}+[x, y] \otimes \frac{c D_{a, b} d+\left(D_{a, b} d\right) c}{2} \\
& -(x, y)_{o} \otimes \frac{c D_{a, b} d-\left(D_{a, b} d\right) c}{2},
\end{aligned}
$$

which is zero by the definition of $[\cdot, \cdot]$ on $\mathscr{E}$ and the fact that $D_{a, b}$ is a derivation.

Case 3. Let $A=x \otimes a, B=y \otimes b, C=z \otimes c \in s l_{3}(k) \otimes S$. We first expand the commutator $[[x \otimes a, y \otimes b], z \otimes c]$ to get

$$
\begin{aligned}
& \frac{1}{3} \operatorname{Tr}(x y) z \otimes D_{a, b} c+\frac{1}{6} \operatorname{Tr}([x, y] z)\{a b+b a, c\}+\frac{1}{6} \operatorname{Tr}\left((x, y)_{o} z\right)\{a b-b a, c\} \\
& \quad+(x y z-y x z-z x y+z y x) \otimes \frac{(a b+b a) c+c(a b+b a)}{4} \\
& \quad+\left(x y z-y x z+z x y-z y x-\frac{2}{3}(\operatorname{Tr}(x y z-y x z)) I_{3}\right. \\
& \quad \otimes \frac{(a b+b a) c-c(a b+b a)}{4} \\
& \quad+(x y z+y x z-z x y-z y x) \otimes \frac{(a b-b a) c+c(a b-b a)}{4} \\
& \quad+\left(x y z+y x z+z x y+z y x-\frac{4}{3} \operatorname{Tr}(x y) z-\frac{2}{3} \operatorname{Tr}(x y z+y x z) I_{3}\right. \\
& \quad \otimes \frac{(a b-b a) c-c(a b-b a)}{4} .
\end{aligned}
$$

We collect terms in the above, and use the shorthand that for $X$, any $3 \times 3$ matrix with entries in $k$, we let $X^{\prime}$ denote the element $X-\frac{1}{3} \operatorname{Tr}(X) I_{3}$, to get

$$
\begin{aligned}
& \frac{1}{3} \operatorname{Tr}(x y) z \otimes\left(D_{a, b} c-(a b-b a) c+c(a b-b a)\right) \\
& \quad+\frac{1}{6} \operatorname{Tr}([x, y] z)\{a b+b a, c\}+\frac{1}{6} \operatorname{Tr}\left((x, y)_{o} z\right)\{a b-b a, c\} \\
& \quad+(x y z)^{\prime}-(y x z)^{\prime}-(z x y)^{\prime}+(z y x)^{\prime} .
\end{aligned}
$$

Recalling the definition of $D_{a, b}$ and the associator $(a, b, c)=(a b) c-a(b c)$ 
the above becomes

$$
\begin{aligned}
& -\operatorname{Tr}(x y) z \otimes(a, b, c)+\frac{1}{6} \operatorname{Tr}([x, y] z)\{a b+b a, c\} \\
& \quad+\frac{1}{6} \operatorname{Tr}\left((x, y)_{o} z\right)\{a b-b a, c\}+(x y z)^{\prime}-(y x z)^{\prime}-(z x y)^{\prime}+(z y x)^{\prime} .
\end{aligned}
$$

Next one writes down the element $J(A, B, C)$ by taking cyclic sums of our last term (2.4). We collect terms in this using associators, properties of $\{\cdot, \cdot\}$ and noting that

$$
\operatorname{Tr}([x, y] z)=\operatorname{Tr}([y, z] x), \quad \operatorname{Tr}\left((x, y)_{o} z\right)=\operatorname{Tr}\left((y, z)_{o} x\right)
$$

to get $J(A, B, C)$ equals

$$
\begin{aligned}
& -\operatorname{Tr}(x y) z \otimes(a, b, c)-\operatorname{Tr}(y z) x \otimes(a, b, c)-\operatorname{Tr}(z x) y \otimes(a, b, c) \\
& +(x y z)^{\prime} \otimes(a, b, c)-(y x z)^{\prime} \otimes(b, a, c)+(z x y)^{\prime} \otimes(c, a, b) \\
& -(z y x)^{\prime} \otimes(c, b, a)+(y z x)^{\prime} \otimes(b, c, a)-(x z y)^{\prime} \otimes(a, c, b) .
\end{aligned}
$$

This equals $X \otimes(a, b, c)$ where

$$
\begin{aligned}
X= & x y z+z x y+y z x+y x z+z y x+x z y \\
& -\operatorname{Tr}(x y) z-\operatorname{Tr}(y z) x-\operatorname{Tr}(z x) y-\operatorname{Tr}(x y z+y x z) I_{3}
\end{aligned}
$$

which is zero by Lemma 2.1. Thus the Jacobi identity is satisfied so $\mathfrak{g}$ is a Lie algebra.

Definition 2.5. We take $\mathscr{E}=\operatorname{Inn}(S)$ in the above construction and denote the resulting Lie algebra by $p s l_{3}(S)$. Thus,

$$
\operatorname{psl}_{3}(S)=\operatorname{Inn}(S) \oplus\left(s l_{3}(k) \otimes S\right) .
$$

If $\mathscr{L}$ is any Lie algebra we let $\mathscr{Z}(\mathscr{L})$ denote its center. Note that if the algebra $S$ is associative then $\operatorname{psl}_{3}(S) \cong s l_{3}(S) / \mathscr{Z}\left(s l_{3}(S)\right)$. This justifies the name $\operatorname{psl}_{3}(S)$ for our algebras.

We now recall the definition of $\Delta$-graded Lie algebras (see [BM], [BGK], [BZ] or $[\mathrm{N}]$ ) and will make use of some facts about such algebras in what follows. We adjust the definition to our particular situation of studying $A_{2}$-graded Lie algebras.

Definition 2.6. Let $\Delta=\left\{0, \pm \alpha_{1}, \pm \alpha_{2}, \pm\left(\alpha_{1}+\alpha_{2}\right)\right\}$ be the root system of type $A_{2}$ with 0 adjoined and let $Q=\mathbb{Z} \alpha_{1} \oplus \mathbb{Z} \alpha_{2}$ be the root lattice. Let $\dot{\mathfrak{g}}$ be the Lie algebra $s l_{3}(k)$ and $\dot{\mathfrak{h}}$ the Cartan subalgebra of $\dot{\mathfrak{g}}$ consisting of diagonal matrices so that $\dot{\mathfrak{h}}$ has basis $h_{1}=E_{11}-E_{22}, h_{2}=E_{22}-E_{33}$ where, as usual, $E_{i j}$ denotes the matrix unit. A Lie algebra $\mathfrak{g}$ over $k$ is $\Delta$-graded (or $A_{2}$-graded) if

(i) $\mathfrak{g}$ has a $Q$-gradation

$$
\mathfrak{g}=\bigoplus_{\alpha \in Q} \mathfrak{g}^{\alpha}
$$

in which $\mathfrak{g}^{\alpha} \neq(0)$ if and only if $\alpha \in \Delta$ (recall $0 \in \Delta$ ),

(ii) the Lie algebra $\dot{\mathfrak{g}}$ is a subalgebra of $\mathfrak{g}$ and if $\dot{\mathfrak{g}}^{\alpha}$ denotes the root space of $\dot{\mathfrak{g}}$ corresponding to $\alpha \in \Delta$ then $\dot{\mathfrak{g}}^{\alpha} \subseteq \mathfrak{g}^{\alpha}$ for all $\alpha \in \Delta$,

(iii) for all $h \in \dot{\mathfrak{h}}, \operatorname{ad}_{\mathfrak{g}}(h)$ act as the scalar $\alpha(h)$ on $\mathfrak{g}^{\alpha}$, and

(iv) $\mathfrak{g}$ is generated by the root spaces $\mathfrak{g}^{\alpha}$ for $\alpha \in \Delta \backslash\{0\}$.

As we are assuming that the characteristic of $k$ is not 2 or 3 then we easily have any $A_{2}$-graded Lie algebra is perfect. 
Corollary 2.7. Let $\mathfrak{g}$ be any of the algebras constructed in Proposition 2.2. Then $\mathfrak{g}$ is $A_{2}$-graded.

Proof. One lets $\mathfrak{g}^{0}=\mathscr{E} \oplus(\dot{\mathfrak{h}} \otimes S)$, and we identify $\dot{\mathfrak{g}}$ with $s l_{3}(k) \otimes 1 \subseteq \mathfrak{g}$, so $\dot{\mathfrak{h}}$ is identified with $\dot{\mathfrak{h}} \otimes 1$. Then let $\mathfrak{g}^{\alpha}=\dot{\mathfrak{g}}^{\alpha} \otimes S$ for $\alpha \in \Delta \backslash\{0\}$. From (a), (b), (c) of Proposition 2.2 we immediately see that (i),(ii),(iii) of Definition 2.6 hold. Moreover, taking $a \in S$ arbitrary and $b=1$ in (a) of Proposition 2.2 yields that $\dot{\mathfrak{h}} \otimes S$ is in the subalgebra of $\mathfrak{g}$ generated by the $\mathfrak{g}^{\alpha}$ with $\alpha \neq 0$. Thus, the term $\frac{1}{3} \operatorname{Tr}(x y)\{a, b\}$ of that formula is also and it follows that (iv) of Definition 2.6 holds, so $\mathfrak{g}$ is $A_{2}$-graded.

Remark 2.8. We have constructed $A_{2}$-graded Lie algebras from the data $(S, \mathscr{E}$, $\{\cdot, \cdot\}, \pi)$ where $S$ is an alternative $k$-algebra with identity, $\{\cdot, \cdot\}: S \times S \rightarrow \mathscr{E}$ a $k$-bilinear map satisfying

(i) $\{a, b\}+\{b, a\}=0$,

(ii) $\{a b, c\}+\{b c, a\}+\{c a, b\}=0$,

(iii) $\mathscr{E}$ is spanned by the elements $\{a, b\}$ for $a, b \in S$,

and where $\pi: \mathscr{E} \rightarrow \operatorname{Inn}(S)$ is a line: $:$ map satisfying $\pi(\{a, b\})=D_{a, b}$ for all $a, b \in S$. There is an obvious notion of isomorphism between such 4tuples. Recalling (1.43)-(1.45) we see that $(\langle S, S\rangle,\langle\cdot, \cdot\rangle, \rho, S)$ is such an object where, as usual, $\rho(\langle a, b\rangle)=D_{a, b}$ and we know $\operatorname{ker} \rho=H C_{1}(S)$. Moreover, by the very definition of $\langle S, S\rangle$ we see that if $(\mathscr{E},\{\cdot, \cdot\}, \pi, S)$ is one of our 4-tuples then there is a morphism

$$
\varphi:(\langle S, S\rangle,\langle\cdot, \cdot\rangle, \rho, S) \rightarrow(\mathscr{E},\{\cdot, \cdot\}, \pi, S)
$$

so in particular there is a surjective $k$-linear map $\varphi:\langle S, S\rangle \rightarrow \mathscr{E}$ such that $\varphi(\langle a, b\rangle)=\{a, b\}$ so $\pi(\varphi(\langle a, b\rangle))=D_{a, b}=\rho(\langle a, b\rangle)$ for all $a, b \in S$. Thus, $\operatorname{ker} \varphi$ is contained in $\operatorname{ker} \rho=H C_{1}(S)$, so since $\langle S, S\rangle / H C_{1}(S) \cong$ $\operatorname{Inn}(S) \cong \mathscr{E} / \operatorname{ker} \pi$ as $k$-space we see that there is a one-to-one correspondence between isomorphism classes of 4-tuples $(S, \mathscr{E},\{\cdot, \cdot\}, \pi)$ and subspaces, $E$ of $H C_{1}(S)$ given by

$$
E \leftrightarrow\left(\langle S, S\rangle / E,\{\cdot, \cdot\}_{E}, \pi_{E}, S\right)
$$

where for $a, b \in S,\{a, b\}_{E}=\langle a, b\rangle+E \in\langle S, S\rangle / E$ and $\pi_{E}(\langle a, b\rangle+E)$ $=D_{a, b}$. To develop the notation a bit more we let $\mathfrak{g}(S, E)=\langle S, S\rangle / E \oplus$ $\left(s l_{3}(k) \otimes S\right)$ be the corresponding Lie algebra so when $E=H C_{1}(S)$ then

$$
\mathfrak{g}(S, E)=\mathfrak{g}\left(S, H C_{1}(S)\right)=p s l_{3}(S)=\operatorname{Inn}(S) \oplus\left(s l_{3}(k) \otimes S\right) .
$$

When $E=(0)$ then

$$
\mathfrak{g}(S, E)=\mathfrak{g}(S,(0))=\langle S, S\rangle \oplus\left(s l_{3}(k) \otimes S\right) .
$$

We can compute the center of the algebras $\mathfrak{g}(S, E)$ as follows.

Corollary 2.9. The center of the algebra $\mathfrak{g}(S, E)$ is

$$
H C_{1}(S) / E \subseteq\langle S, S\rangle / E \subseteq\langle S, S\rangle / E \oplus\left(s l_{3}(k) \otimes S\right)=\mathfrak{g}(S, E) .
$$

In particular, $\mathscr{Z}\left(\right.$ psl $\left._{3}(S)\right)=(0)$.

Proof. Since $\mathfrak{g}(S, E)$ is an $A_{2}$-graded Lie algebra then clearly $\mathscr{Z}(\mathfrak{g}(S, E)) \subseteq$ $\mathfrak{g}(S, E)^{0}=\langle S, S\rangle / E \oplus(\dot{\mathfrak{h}} \otimes S)$. Assume that $z=D+\sum_{i \in I} x_{i} \otimes s_{i} \in \mathscr{Z}(\mathfrak{g}(S, E))$ where $D \in\langle S, S\rangle / E,\left\{s_{i}\right\}_{i \in I}$ is a $k$-basis of $S$ and $x_{i} \in \mathfrak{h}$ for $i \in I$ with $x_{i}=$ 
0 for all but a finite number of indices $i \in I$. Then for $x \in \dot{\mathfrak{g}}, 0=[z, x \otimes 1]=$ $\sum_{i \in I}\left[x_{i}, x\right] \otimes s_{i}$ so that $\left[x_{i}, x\right]=0$ for all $i \in I$ and all $x \in \dot{\mathfrak{g}}$. This implies that $x_{i}=0$ for all $i \in I$ so that $z=D \in\langle S, S\rangle / E$. Then for any $x \in \dot{\mathfrak{g}}, a \in S$ we have in $\mathfrak{g}(S, E)$ that $0=[D, x \otimes a]=x \otimes \pi_{E}(D)(a)$ so that $\pi_{E}(D)=0$. Now if $D=\sum_{i}\left\langle a_{i}, b_{i}\right\rangle+E$ then $\pi_{E}(D)=\sum_{i} D_{a_{i}}, b_{i}$ so we get $\sum_{i}\left\langle a_{i}, b_{i}\right\rangle \in H C_{1}(S)$ and $D \in H C_{1}(S) / E$. Clearly $H C_{1}(S) / E \subseteq \mathscr{Z}(\mathfrak{g}(S, E))$.

For any perfect Lie algebra $\mathfrak{g}$ we let $\hat{\mathfrak{g}}$ denote its universal central extension. We are going to show that $\mathfrak{g}(S,(0))=p \widehat{l_{3}(S)}$ and it follows from this that in fact $\mathfrak{g}(S,(0))=\mathfrak{g}(\widehat{S, E})$ for any subset $E$ of $H C_{1}(S)$. It also follows that $H_{2}(\mathfrak{g}(S, E), k) \cong E$. In order to prove this it is convenient to use the Steinberg Lie algebra which we now introduce.

Definition 2.10. The Steinberg Lie algebra, $s t_{3}(S)$, is defined to be the Lie algebra over $k$ given by

$$
\text { generators: } u_{i j}(a) \text {, for } 1 \leq i \neq j \leq 3, a \in S \text {, }
$$

and

$$
\text { relations: }\left[u_{i j}(a), u_{k l}(b)\right]=\left\{\begin{array}{l}
u_{i l}(a b) \text { if } i \neq l, j=k, \\
0 \text { if } i \neq l, j \neq k .
\end{array}\right.
$$

One knows from Proposition 3.17 of [BM] that $s t_{3}(S)$ is an $A_{2}$-graded Lie algebra whose associated coordinate algebra is $S$ and moreover that $s t_{3}(S)$ is centrally closed (see [G] or [BM]). It follows that $p s \widehat{l_{3}(S)} \cong s t_{3}(S)$.

We let $H_{i j}(a, b)=\left[u_{i j}(a), u_{j i}(b)\right]$ for $1 \leq i \neq j \leq 3$ and $a, b \in S$ so that

$$
H_{i j}(a, b)=-H_{j i}(b, a) .
$$

By the Jacobi identity we get

$$
\begin{aligned}
H_{i j}(a b, c) & =\left[u_{i j}(a b), u_{j i}(c)\right]=\left[\left[u_{i k}(a), u_{k j}(b)\right], u_{j i}(c)\right] \\
& =\left[u_{i k}(a), u_{k i}(b c)\right]-\left[u_{k j}(b),\left[u_{i k}(a), u_{j i}(c)\right]\right] \\
& =H_{i k}(a, b c)+H_{k j}(b, c a)
\end{aligned}
$$

for $a, b, c \in S$ and distinct indices $i, j, k$.

Taking $a=b=1$ in (2.11) we get

$$
H_{i j}(1, c)=H_{i k}(1, c)+H_{k j}(1, c),
$$

interchanging $j$ and $k$ gives

$$
H_{i k}(1, c)=H_{i j}(1, c)+H_{j k}(1, c),
$$

combining these yields

$$
H_{k j}(1, c)=-H_{j k}(1, c)=H_{k j}(c, 1),
$$

for distinct $j$ and $k$ and $c \in S$.

Now let $\{j, k\}=\{2,3\}$ and consider the element $H_{1 j}(a, b)-H_{1 j}(1, b a)$. By (2.11) this equals $H_{1 k}(a, b)+H_{k j}(1, b a)-H_{1 j}(1, b a)$ so by using (2.12) this becomes $H_{1 k}(a, b)-\left(H_{j k}(1, b a)+H_{1 j}(1, b a)\right)$ which by $(2.11)$ again is just $H_{1 k}(a, b)-H_{i k}(1, b a)$. We define $h(a, b)$ by

$$
h(a, b)=H_{1 j}(a, b)-H_{1 j}(1, b a),
$$

and note this does not depend on the choices of $\{2,3\}$ by the above computation. 
Lemma 2.14. For $a, b, c \in S$ we have

(1) $h(a, b)+h(b, a)=0$, and

(2) $h(a b, c)+h(b c, a)+h(c a, b)=H_{12}(1,(a, b, c))+H_{13}(1,(a, b, c))$.

Proof. Since $H_{12}(a b, c)=H_{13}(a, b c)+H_{32}(b, c a)$ then

$h(a b, c)+H_{12}(1, c(a b))=H_{12}(a b, c)=h(a, b c)+H_{13}(\mathbf{i},(b c) a)+H_{32}(b, c a)$.

Also, since

$$
\begin{aligned}
H_{32}(b, c a) & =H_{31}(b, c a)+H_{12}(1,(c a) b) \\
& =-H_{13}(c a, b)+H_{12}(1,(c a) b),
\end{aligned}
$$

we have

$$
\begin{aligned}
& h(a b, c)+H_{12}(1, c(a b)) \\
& \quad=h(a, b c)+H_{13}(1,(b c) a)-H_{13}(c a, b)+H_{12}(1,(c a) b) \\
& \quad=h(a, b c)+H_{13}(1,(b c) a)-h(c a, b)-H_{13}(1, b(c a))+H_{12}(1,(c a) b) .
\end{aligned}
$$

This gives us

$$
h(a b, c)-h(a, b c)+h(c a, b)=H_{12}(1,(c, a, b))+H_{13}(1,(b, c, a)) .
$$

Setting $a=1$ and using that $h(1, b c)=0$ we have $h(b, c)+h(c, b)=0$ which proves (1). Also, we have

$$
h(a b, c)+h(b c, a)+h(c a, b)=H_{12}(1,(a, b, c))+H_{13}(1,(a, b, c))
$$

which is (2).

We now define $T(a, b)$ by

$$
T(a, b)=3 h(a, b)-H_{12}(1, a b-b a)-H_{13}(1, a b-b a),
$$

for $a, b \in S$.

Lemma 2.16. For $a, b, c \in S$ we have

$$
\begin{aligned}
& T(a, b)+T(b, a)=0, \text { and } \\
& T(a b, c)+T(b c, a)+T(c a, b)=0 .
\end{aligned}
$$

Proof. (1) is clear from the definition of $T(a, b)$ and the corresponding property for $h(a, b)$. As for (2) we have by (2) of Lemma 2.14 that

$$
\begin{aligned}
T(a b, c) & +T(b c, a)+T(c a, b) \\
= & 3 H_{12}(1,(a, b, c))+3 H_{13}(1,(a, b, c)) \\
& -H_{12}(1,(a b) c-c(a b))-H_{13}(1,(a b) c-c(a b)) \\
& -H_{12}(1,(b c) a-a(b c))-H_{13}(1,(b c) a-a(b c)) \\
& -H_{12}(1,(c a) b-b(c a))-H_{13}(1,(c a) b-b(c a)) \\
= & 3 H_{12}(1,(a, b, c))+3 H_{13}(1,(a, b, c)) \\
& -H_{12}(1,(a, b, c)+(b, c, a)+(c, a, b)) \\
& -H_{13}(1,(a, b, c)+(b, c, a)+(c, a, b)) \\
= & 0
\end{aligned}
$$

as desired. 
In order to show $\mathfrak{g}(S,(0))$ is isomorphic to $s t_{3}(S)$ we let $\varphi: s_{3}(S) \rightarrow$ $\mathfrak{g}(S,(0))$ be the homomorphism defined by $\varphi\left(u_{i j}(a)\right)=E_{i j} \otimes a$, for $1 \leq i \neq$ $j \leq 3$ and $a \in S$. Here we are using the universal property inherent in the definition of $s_{3}(S)$ and the trivial fact that the elements $E_{i j} \otimes a$ satisfy the necessary relations. Since $\mathfrak{g}(S,(0))$ is an $A_{2}$-graded Lie algebra the elements $E_{i j} \otimes a$ generate $\mathfrak{g}(S,(0))$ so that $\varphi$ is surjective. Moreover, letting $u_{i j}(S)=$ $\left\{u_{i j}(a) \mid a \in S\right\}$, we see that $\varphi$ is one-to-one when restricted to $\bigoplus_{1 \leq i \neq j \leq 3} u_{i j}(S)$.

Next we let $s t_{3}(S)^{0}=\sum_{1 \leq i \neq j \leq 3}\left[u_{i j}(S), u_{j i}(S)\right]$ so that

$$
s t_{3}(S)=s t_{3}(S)^{0} \oplus\left(\bigoplus_{1 \leq i \neq j \leq 3} u_{i j}(S)\right) .
$$

Noting that for $i \neq j$ we have

$$
\varphi\left(H_{i j}(a, 1)\right)=\left[E_{i j} \otimes a, E_{j i} \otimes 1\right]=\left(E_{i i}-E_{j j}\right) \otimes a,
$$

we see if $H$ is the span of all elements $H_{i j}(a, 1)$ for $1 \leq i \neq j \leq 3$ and $a \in S$, then $\varphi(H)=\dot{h} \otimes S$. Also, recalling (2.11) and (2.12) we see that $H=H_{12}(S, 1)+H_{23}(S, 1)$ so since $\varphi(H)=\dot{\mathfrak{h}} \otimes S \cong S \oplus S$ we see that $\varphi$ is one-to-one when restricted to $H$.

Finally put $T$ equal to the span of all elements $T(a, b)$ for $a, b \in S$. Since $T(a, b)$ equals

$$
3\left(H_{12}(a, b)-H_{12}(1, b a)\right)-H_{12}(1, a b-b a)-H_{13}(1, a b-b a),
$$

then since

$$
\varphi\left(H_{12}(a, b)\right)=\frac{1}{3}\langle a, b\rangle+\left(E_{11}-E_{22}\right) \otimes \frac{a b+b a}{2}+\left(E_{12}, E_{21}\right)_{o} \otimes \frac{a b-b a}{2},
$$

and

$$
\varphi\left(H_{13}(a, b)\right)=\frac{1}{3}\langle a, b\rangle+\left(E_{11}-E_{33}\right) \otimes \frac{a b+b a}{2}+\left(E_{13}, E_{31}\right)_{o} \otimes \frac{a b-b a}{2},
$$

we get that

$$
\begin{aligned}
\varphi(T(a, b))= & \langle a, b\rangle+3\left(E_{11}-E_{22}\right) \otimes \frac{a b+b a}{2}+3\left(E_{12}, E_{21}\right)_{o} \\
& \otimes \frac{a b-b a}{2}-3\left(E_{11}-E_{22}\right) \otimes b a-\left(E_{11}-E_{22}\right) \\
& \otimes(a b-b a)-\left(E_{11}-E_{33}\right) \otimes(a b-b a) \\
= & \langle a, b\rangle .
\end{aligned}
$$

By Lemma 2.16 and the universal property inherent in the definition of $\langle S, S\rangle$ we see that $\varphi$ is one-to-one when restricted to $T$.

From the definition of $T(a, b)$ we find that $H_{12}(a, b), H_{13}(a, b) \in T+H$ for all $a, b \in S$ so by $(2.11)$ we see $H_{23}(a, b) \in T+H$ and hence $s_{3}(S)^{0}=$ $T+H$. Moreover,

$$
\varphi\left(s t_{3}(S)^{0}\right)=\varphi(T)+\varphi(H)=\langle S, S\rangle+\dot{\mathfrak{h}} \otimes S=\langle S, S\rangle \oplus(\dot{\mathfrak{h}} \otimes S)
$$

so that $s t_{3}(S)^{0}=T \oplus H$ and hence $\varphi$ is one-to-one on $\operatorname{st}_{3}(S)^{0}$ and so is an isomorphism of $s_{3}(S)$ and $\mathfrak{g}(S,(0))$. This establishes most of our next result. 
Theorem 2.17. If $\mathfrak{g}$ is an $A_{2}$-graded Lie algebra over $k$ with coordinate algebra $S$ which is an alternative algebra with identity over $k$ then there is a subset $E$ of $H C_{1}(S)$ such that $\mathfrak{g}$ is isomorphic to $\mathfrak{g}(S, E)$. Each of the algebras $\mathfrak{g}(S, E)$ is a central extension of $\mathfrak{g}\left(S, H C_{1}(S)\right)=p s l_{3}(S)$ and the universal central extension of each of these is the algebra $\mathfrak{g}(S,(0))$ which is isomorphic to $\mathrm{st}_{3}(S)$.

Proof. We already know that $\mathfrak{g}(S,(0))$ and $s t_{3}(S)$ is isomorphic. The rest of the result follows from this and the results in Section 3 of [BM].

We next want to compute the space of invariant symmetric forms on the algebras $\mathfrak{g}(S, E)$. Our result generalizes the situation when the coordinate algebra is associative, which is dealt with in Lemma 2.8 of [BGK]. Recall the following definition; see [BGK] or [BeK].

Definition 2.18. If $\mathfrak{g}$ is a Lie algebra over $k$ we let $J$ be the subspace of $\mathfrak{g} \otimes \mathfrak{g}$ spanned by all elements of the form $x \otimes y-y \otimes x,[x, y] \otimes z-x \otimes[y, z]$ for all $x, y, z \in \mathfrak{g}$. Let $V(\mathfrak{g})$ be the factor space of $\mathfrak{g} \otimes \mathfrak{g}$ by $J$ so that $V(\mathfrak{g})=\mathfrak{g} \otimes \mathfrak{g} / J$.

Note that the dual space, $V(\mathfrak{g})^{*}$, is just the space of invariant symmetric bilinear forms on $\mathfrak{g}$. Moreover, if $\mathfrak{g}$ is perfect then any invariant form is automatically symmetric so in this case we can leave out the elements $x \otimes y-$ $y \otimes x$ in the definition of $J$.

For our alternative $k$-algebra $S$ we let $[S, S]$ (resp. $(S, S, S)$ ) be the space spanned by all the commutators $[a, b]=a b-b a$ ( resp. associators $(a, b, c)=(a b) c-a(b c))$ for $a, b, c \in S$, and define

$$
\mathscr{I}(S)=[S, S]+(S, S, S) .
$$

Let $\mathfrak{g}$ be one of our $A_{2}$-graded Lie algebras with coordinate algebra $S$. We are going to show that $V(\mathfrak{g}) \cong S / \mathscr{I}(S)$. For notation we let $x \times y=x \otimes y+J$ in $V(\mathfrak{g})$ so that $x \times y=y \times x,[x, y] \times z=x \times[y, z]$ for $x, y, z \in \mathfrak{g}$. Also let $E_{i j}(r)$ denote the element $E_{i j} \otimes r$ in $\mathfrak{g}$.

Lemma 2.20. $V(\mathfrak{g})$ is spanned by the elements $E_{12}(1) \times E_{21}(r)$ for $r \in S$. Moreover, $E_{12}(1) \times E_{21}(r)=0$ if $r \in \mathscr{I}(S)$.

Proof. The usual argument using the two properties of symmetry and invariance together with the fact that $\mathfrak{g}$ is $A_{2}$-graded shows that $\mathfrak{g}^{\alpha} \times \mathfrak{g}^{\beta}=(0)$ for $\alpha, \beta \in$ $\Delta, \alpha+\beta \neq 0$. It easily follows from this that $V(\mathfrak{g})$ is spanned by elements $E_{i j}(r) \times E_{j i}(s)$ for $r, s \in S, 1 \leq i \neq j \leq 3$. Since $E_{13}(r s)=\left[E_{12}(r), E_{23}(s)\right]$ then

$$
\begin{aligned}
& E_{13}(r s) \times E_{31}(t)=\left[E_{12}(r), E_{23}(s)\right] \times E_{31}(t) \\
& \quad=E_{12}(r) \times\left[E_{23}(s), E_{31}(t)\right]=E_{12}(r) \times E_{21}(s t) .
\end{aligned}
$$

That is

$$
E_{13}(r s) \times E_{31}(t)=E_{12}(r) \times E_{21}(s t), \text { for } r, s, t \in S .
$$

Taking $s=1$ yields

$$
E_{13}(r) \times E_{31}(t)=E_{12}(r) \times E_{21}(t), \text { for } r, t \in S .
$$

Also,

$$
\begin{aligned}
E_{12}(r) \times E_{21}(t)=E_{31}(t) \times E_{13}(r) \\
=\left[E_{32}(t), E_{32}(1)\right] \times E_{13}(r)=E_{32}(t) \times E_{23}(r) .
\end{aligned}
$$


so that

$$
E_{12}(r) \times E_{21}(t)=E_{23}(r) \times E_{32}(t), \text { for } r, t \in S .
$$

We also have that $E_{12}(s) \times E_{21}(t)=E_{12}(1) \times E_{21}(s t)$ by taking $r=1$ in (2.21) and using (2.22). It now follows that $V(\mathfrak{g})$ is spanned by the elements $E_{12}(1) \times E_{21}(r)$ for $r \in S$.

We have, by (2.23),

$$
\begin{aligned}
& E_{12}(1) \times E_{21}(r t)=E_{12}(r) \times E_{21}(t)=E_{21}(t) \times E_{12}(r) \\
& =\left[E_{23}(1), E_{31}(t)\right] \times E_{12}(r)=E_{23}(1) \times E_{32}(t r)=E_{12}(1) \times E_{21}(t r) .
\end{aligned}
$$

Thus, $E_{12}(1) \times E_{21}(r)=0$ if $r \in[S, S]$.

Finally we have, by (2.21) and (2.22),

$$
\begin{gathered}
E_{12}(1) \times E_{21}((r s) t)=E_{12}(r s) \times E_{21}(t)=E_{13}(r) \times E_{31}(s t) \\
=E_{12}(r) \times E_{21}(s t)=E_{12}(1) \times E_{21}(r(s t)) .
\end{gathered}
$$

This shows $E_{12}(1) \times E_{21}(r)=0$ if $r \in(S, S, S)$.

Our next result just deals with $S$.

Lemma 2.24. Let $a, b, c, d, e, f \in S$. Then

(1) $\left(D_{a, b} c\right) d-\left(D_{c, d} a\right) b=0(\bmod \mathscr{I}(S))$.

(2) $\left(\left[D_{a, b}, D_{c, d}\right] e\right) f+\left(\left[D_{e, f}, D_{c, d}\right] a\right) b=0(\bmod \mathscr{I}(S))$.

Proof. To prove (1) we compute as follows.

$$
\begin{gathered}
\left(D_{a, b} c\right) d-\left(D_{c, d} a\right) b=((a b-b a) c) d-(c(a b-b a)) d-3((a b) c-a(b c)) d \\
-((c d-d c) a) b+(a(c d-d c)) b+3((c d) a-c(d a)) b .
\end{gathered}
$$

Using that $(S, S, S)+[S, S] \subseteq \mathscr{I}(S)$ we find this is congruent modulo $\mathscr{I}(S)$ to the element

$$
\begin{aligned}
& (a b-b a)(c d)-(d c)(a b-b a)-3(a b)(c d)+3(d a)(b c) \\
& -(c d-d c)(a b)+(b a)(c d-d c)+3(c d)(a b)-3(b c)(d a)
\end{aligned}
$$

Because $[S, S] \subseteq \mathscr{I}(S)$ we find this last element belongs to $\mathscr{I}(S)$.

Next note that the element on the left-hand side of $(2)$ is

$$
\begin{aligned}
& \left(D_{D_{a, b} c, d} e+D_{c, D_{a, b} d} e\right) f+\left(D_{D_{e, f} c, d} a+D_{c, D_{e, f} d} a\right) b \\
& \quad=-\left(D_{d, D_{a, b} c} e\right) f+\left(D_{c, D_{a, b} d} e\right) f-\left(D_{d, D_{e, f} c} a\right) b+\left(D_{c, D_{e, f}} a\right) b .
\end{aligned}
$$

Using (1) we find that modulo $\mathscr{I}(S)$ this is congruent to

$$
-\left(D_{e, f} d\right)\left(D_{a, b} c\right)+\left(D_{e, f} \mathcal{c}\right)\left(D_{a, b} d\right)-\left(D_{a, b} d\right)\left(D_{e, f} \mathcal{C}\right)+\left(D_{a, b} c\right)\left(D_{e, f} d\right)
$$

which is clearly in $\mathscr{F}(S)$.

We can now prove that $V(\mathfrak{g}) \cong S / \mathscr{I}(S)$.

Proposition 2.25. Let $\mathfrak{g}$ be one of the algebras $\mathfrak{g}(S, E)$. Then $V(\mathfrak{g}) \cong S / \mathscr{I}(S)$. 
Proof. One knows (see the remark after Note 1.27 in $[\mathrm{BeK}]$ ) that if $0 \rightarrow \mathscr{Z} \rightarrow$ $\hat{\mathfrak{g}} \rightarrow \mathfrak{g} \rightarrow 0$ is a short exact sequence of Lie algebras with $\mathscr{Z}$ central in $\hat{\mathfrak{g}}$ and $\hat{\mathfrak{g}}$ perfect then $V(\hat{\mathfrak{g}}) \cong V(\mathfrak{g})$. Thus, it is enough for us to assume that

$$
\mathfrak{g}=\mathfrak{g}\left(S, H C_{1}(S)\right)=p s l_{3}(S)=\operatorname{Inn}(S) \oplus\left(s l_{3}(k) \otimes S\right)
$$

Define a linear mapping $\lambda: p s l_{3}(S) \otimes p s l_{3}(S) \rightarrow S / \mathscr{I}(S)$, by the following formulas:

$$
\begin{aligned}
& \lambda((x \otimes a) \otimes(y \otimes b))=\operatorname{Tr}(x y) a b+\mathscr{I}(S), \\
& \lambda\left(D_{a, b} \otimes(x \otimes c)\right)=\lambda\left((x \otimes c) \otimes D_{a, b}\right)=0, \text { and } \\
& \lambda\left(D_{a, b} \otimes D_{c, d}\right)=3\left(D_{a, b} c\right) d+\mathscr{I}(S),
\end{aligned}
$$

for $x, y \in s l_{3}(k), a, b, c, d \in S$.

Note that by Lemma 2.24(1) and the definition of $\lambda$ we have that $\lambda$ is symmetric in the sense that $\lambda\left(l_{1} \otimes l_{2}-l_{2} \otimes l_{1}\right)=0$ for any $l_{1}, l_{2} \in \mathfrak{g}$. We next show $\lambda\left(\left[l_{1}, l_{2}\right] \otimes l_{3}-l_{1} \otimes\left[l_{2}, l_{3}\right]\right)=0$ for any $l_{1}, l_{2}, l_{3} \in \mathfrak{g}$ by considering the following five cases.

Case 1. $l_{1}, l_{2}, l_{3} \in \operatorname{Inn}(S)$. We have for $a, b, c, d, e, f \in S$ that

$$
\begin{gathered}
\lambda\left(\left[D_{a, b}, D_{c, d}\right] \otimes D_{e, f}-D_{a, b} \otimes\left[D_{c, d}, D_{e, f}\right]\right) \\
\quad=\lambda\left(\left[D_{a, b}, D_{c, d}\right] \otimes D_{e, f}\right)-\lambda\left(\left[D_{e, f}, D_{c, d}\right] \otimes D_{a, b}\right) \\
\quad=3\left(\left[D_{a, b}, D_{c, d}\right] e\right) f+3\left(\left[D_{e, f}, D_{c, d}\right] a\right) b+\mathscr{I}(S)
\end{gathered}
$$

and this is 0 by Lemma $2.24(2)$.

Case 2. Two of $l_{1}, l_{2}, l_{3}$ are in $\operatorname{Inn}(S)$ and one is in $s l_{3}(k) \otimes S$. Here we obviously have that $\lambda\left(\left[l_{1}, l_{2}\right] \otimes l_{3}-l_{1} \otimes\left[l_{2}, l_{3}\right]\right)=0$ by definition of $\lambda$.

Case 3. $l_{1} \in \operatorname{Inn}(S), l_{2}, l_{3} \in S l_{3}(k) \otimes S$. We compute as follows:

$$
\begin{aligned}
& \lambda\left(\left[D_{a, b}, x \otimes c\right] \otimes(y \otimes d)-D_{a, b} \otimes[x \otimes c, y \otimes d]\right) \\
&= \lambda\left(\left(x \otimes D_{a, b} c\right) \otimes(y \otimes d)\right) \\
& \quad-\lambda\left(D_{a, b} \otimes\left(\frac{1}{3} \operatorname{Tr}(x y) D_{c, d}+[x, y] \otimes \frac{c d+d c}{2}+(x, y)_{o} \otimes \frac{c d-d c}{2}\right)\right) \\
&= \operatorname{Tr}(x y)\left(D_{a, b} c\right) d-\frac{1}{3} \operatorname{Tr}(x y) \lambda\left(D_{a, b} \otimes D_{c, d}\right)+\mathscr{I}(S) \\
&= 0(\bmod \mathscr{I}(S)) .
\end{aligned}
$$

Case $3^{\prime} . l_{1}, l_{3} \in s l_{3}(k) \otimes S$ and $l_{2} \in \operatorname{Inn}(S)$,

$$
\begin{aligned}
\lambda(-x & \otimes D a, y \otimes b)-\lambda(x \otimes a, y \otimes D b) \\
& =-\operatorname{Tr}(x y)(D a) b-\operatorname{Tr}(x y) a D b+\mathscr{I}(S) \\
& =-\operatorname{Tr}(x y) D(a b)+\mathscr{I}(S) \\
& =0(\bmod \mathscr{I}(S))
\end{aligned}
$$

as one can easily check that $D \mathscr{I}(S) \subseteq \mathscr{I}(S)$. 
Case 4. $l_{1}, l_{2}, l_{3} \in s l_{3}(k) \otimes S$. Here we have $\lambda([x \otimes a, y \otimes b] \otimes(z \otimes c)-(x \otimes a) \otimes[y \otimes b, z \otimes c])$

$$
\begin{aligned}
= & \lambda\left(\left(\frac{1}{3} \operatorname{Tr}(x y) D_{a, b}+[x, y] \otimes \frac{a b+b a}{2}+(x, y)_{o} \otimes \frac{a b-b a}{2}\right) \otimes(z \otimes c)\right) \\
& -\lambda\left((x \otimes a) \otimes\left(\frac{1}{3} \operatorname{Tr}(y z) D_{b, c}+[y, z] \otimes \frac{b c+c b}{2}+(y, z)_{o} \otimes \frac{b c-c b}{2}\right)\right) \\
= & \frac{1}{2} \operatorname{Tr}([x, y] z)((a b+b a) c)+\frac{1}{2} \operatorname{Tr}\left((x, y)_{o} z\right)((a b-b a) c) \\
& -\frac{1}{2} \operatorname{Tr}([x, y] z)(a(b c+c b))-\frac{1}{2} \operatorname{Tr}\left(x(y, z)_{o}\right)(a(b c-c b))+\mathscr{I}(S) \\
= & \frac{1}{2} \operatorname{Tr}([x, y] z)((a b) c+(b a) c-a(b c)-a(c b)) \\
& \left.+\frac{1}{2} \operatorname{Tr}\left((x, y)_{o} z\right)((a b) c-(b a) c-a(b c)+a(c b))\right)+\mathscr{J}(S) \\
= & \frac{1}{2} \operatorname{Tr}([x, y] z)((b a) c-(c b) a)+\frac{1}{2} \operatorname{Tr}\left((x, y)_{o} z\right)(-(b a) c+(c b) a)+\mathscr{I}(S) \\
= & \frac{1}{2} \operatorname{Tr}([x, y] z)((b a) c-c(b a))+\frac{1}{2} \operatorname{Tr}\left((x, y)_{o} z\right)(-(b a) c+c(b a))+\mathscr{I}(S) \\
= & 0(\bmod \mathscr{I}(S)) .
\end{aligned}
$$

It now follows that $\lambda$ induces a map, which we also call $\lambda$, of $V(\mathfrak{g})$ onto $S / \mathscr{I}(S)$ and moreover $\lambda\left(E_{12}(1) \times E_{21}(r)\right)=r+\mathscr{I}(S)$. Next, define a map $\tau: S \rightarrow V(\mathfrak{g})$ by $\tau(r)=E_{12}(1) \times E_{21}(r)$ and note by Lemma $2.20 \tau$ induces a surjective map of $S / \mathscr{I}(S)$ onto $V(\mathfrak{g})$ which clearly satisfies $\lambda \circ \tau=$ id as desired.

When $S=\mathfrak{A}$ is the alternative torus then we know that $\mathfrak{A}^{\prime}=\{x \in \mathfrak{A} \mid t(x)=$ $0\}=[\mathfrak{A}, \mathfrak{A}]=(\mathfrak{A}, \mathfrak{A}, \mathfrak{A})$ so that $\mathscr{I}(\mathfrak{A})=\bigoplus_{i=2}^{8} K u_{i}$ and hence for $\mathfrak{g}=\mathfrak{g}(\mathfrak{A}, E)$ we have

$$
V(\mathfrak{g}) \cong \mathfrak{A} / \mathscr{I}(\mathfrak{A}) \cong K=k\left[t_{1}^{ \pm 1}, \ldots, t_{\nu}^{ \pm 1}\right] .
$$

Remark 2.27. If $\varphi \in(S / \mathscr{I}(S))^{*}$ we define a bilinear form $F_{\varphi}: p s l_{3}(S) \times$ $\operatorname{psl}_{3}(S) \rightarrow k$ by the formulas

$$
\begin{aligned}
& F_{\varphi}((x \otimes a),(y \otimes b))=\operatorname{Tr}(x y) \varphi(a b+\mathscr{I}(S)), \\
& F_{\varphi}\left(D_{a, b},(x \otimes c)\right)=F_{\varphi}\left((x \otimes c), D_{a, b}\right)=0, \text { and } \\
& F_{\varphi}\left(D_{a, b}, D_{c, d}\right)=\varphi\left(3\left(D_{a, b} c\right) d+\mathscr{I}(S)\right),
\end{aligned}
$$

for $a, b, c, d \in S, x, y \in s_{3}(k)$. Then $F_{\varphi}$ is the symmetric invariant bilinear form in $V\left(p s l_{3}(S)\right)^{*}$ associated with the functional $\varphi \in(S / \mathscr{I}(S))^{*}$ as in the previous proposition. For $E \subseteq H C_{1}(S)$ we let $\psi: \mathfrak{g}(S, E) \rightarrow p s l_{3}(S)$ be the natural surjective homomorphism and lift $F_{\varphi}$ to a form on $\mathfrak{g}(S, E)$, which we also denote by $F_{\varphi}$, by

$$
F_{\varphi}\left(l_{1}, l_{2}\right)=F_{\varphi}\left(\psi\left(l_{1}\right), \psi\left(l_{2}\right)\right), \quad l_{1}, l_{2} \in \mathfrak{g}(S, E) .
$$

As in the Proposition we have $V(\mathfrak{g}(S, E))^{*}=\left\{F_{\varphi} \mid \varphi \in(S / \mathscr{I}(S))^{*}\right\}$.

We now make some remarks about derivations of $p s l_{3}(S)$. The results and proofs are exactly as in the case when the coordinate algebra is associative, see 
(2.10)-(2.17) of [BGK], so we will be brief and just recall them here in the following remark.

Remark 2.29. Let $\mathscr{M}$ denote the derivation algebra of $\mathfrak{g}=p s l_{3}(S)$ and let for $\alpha \in \Delta \mathscr{M}^{\alpha}=\left\{D \in \mathscr{M} \mid D\left(\mathfrak{g}^{\beta}\right) \subseteq \mathfrak{g}^{\alpha+\beta}\right.$ for all $\left.\beta \in \Delta\right\}$ Then $\mathscr{M}=\bigoplus_{\alpha \in \Delta} \mathscr{M}^{\alpha}$, and it follows just as in [BGK] that $\mathscr{M}=\operatorname{Inn}\left(\mathrm{psl}_{3}(S)\right)+\mathscr{M}^{0}$, where, of course, $\operatorname{Inn}\left(\operatorname{psl}_{3}(S)\right)$ is the ideal of inner derivations in the Lie algebra $\mathscr{M}$. Also, if $D \in \operatorname{Der}_{k}(S)$ then $D$ gives rise to a derivation of $p s l_{3}(S)$ by the following formulas:

$$
\begin{aligned}
& D(x \otimes a)=x \otimes D a, \\
& D\left(D_{a, b}\right)=\left[D, D_{a, b}\right]=D_{D a, b}+D_{a, D b},
\end{aligned}
$$

for $a, b \in S, x \in s l_{3}(k)$. In this way we have $\operatorname{Der}_{k}(S) \subseteq \mathscr{M}^{0}$ and in fact we have $\mathscr{M}=\operatorname{Inn}\left(p s l_{3}(S)\right)+\operatorname{Der}_{k}(S)$, and $\operatorname{Inn}_{k}(S) \subseteq \operatorname{Inn}\left(p s l_{3}(S)\right) \cap \operatorname{Der}_{k}(S)$.

Recalling the form $F_{\varphi}$ for $\varphi \in(S / \mathscr{I}(S))^{*}$ in Remark 2.27 we make the following definition.

Definition 2.30. For $\varphi \in(S / \mathscr{I}(S))^{*}$ let

$$
\mathscr{M}_{\varphi}\left(\operatorname{psl}_{3}(S)\right)=\mathscr{M}_{\varphi}=\left\{D \in \mathscr{M} \mid D \text { is skew-symmetric w.r.t } F_{\varphi}\right\} .
$$

Similarly, we can define $\mathscr{M}_{\varphi}(\mathfrak{g}(S, E))$ for any subset $E$ of $H C_{1}(S)$ as we know $F_{\varphi}$ gives rise to a symmetric invariant form on $\mathfrak{g}(S, E)$. Notice that we always have the inner derivations $\operatorname{Inn}\left(\operatorname{psl}_{3}(S)\right)$ belong to $\mathscr{M}_{\varphi}$ because the form $F_{\varphi}$ is invariant. Thus, $\mathscr{M}_{\varphi}=\operatorname{Inn}\left(p s l_{3}(S)\right)+\tilde{\mathscr{M}}_{\varphi}$ where

$$
\tilde{\mathscr{M}}_{\varphi}=\left\{D \in \operatorname{Der}_{k}(S) \subseteq \mathscr{M} \mid D \in \mathscr{M}_{\varphi}\right\} .
$$

Lemma 2.32. (1) $\tilde{\mathscr{M}}_{\varphi}=\left\{D \in \operatorname{Der}_{k}(S) \mid \varphi(D a+\mathscr{I}(S))=0\right.$ for all $\left.a \in S\right\}$.

(2) $\mathscr{M}_{\varphi}\left(p s \widehat{l_{3}(S)}\right) \cong \mathscr{M}_{\varphi}\left(p s l_{3}(S)\right)$.

Proof. From Remark 2.27, We have for $D \in \mathscr{M}$,

$$
\begin{aligned}
F_{\varphi}( & (x \otimes a), y \otimes b)+F_{\varphi}(x \otimes, D(y \otimes b)) \\
& =F_{\varphi}(x \otimes D a, y \otimes b)+F_{\varphi}(x \otimes a, y \otimes D b) \\
& =\operatorname{Tr}(x y) \varphi((D a) b+a D b+\mathcal{I}(S)) \\
& =\operatorname{Tr}(x y) \varphi(D(a b)+\mathscr{I}(S)) .
\end{aligned}
$$

Also, we have

$$
F_{\varphi}\left(D\left(D_{a, b}\right), x \otimes c\right)+F_{\varphi}\left(D_{a, b}, D(x \otimes c)\right)=0
$$

and it is easy to see that we have

$$
\begin{aligned}
& F_{\varphi}\left(D\left(D_{a, b}\right), D_{c, d}\right)+F_{\varphi}\left(D_{a, b}, D\left(D_{c, d}\right)\right) \\
& \quad=3 \varphi\left(\left(D_{D a, b} c\right) d+\left(D_{a, D b} c\right) d+\left(D_{a, b}(D c)\right) d+\left(D_{a, b} c\right)(D d)+\mathscr{I}(S)\right) \\
& \quad=3 \varphi\left(D\left(\left(D_{a, b} c\right) d\right)+\mathscr{I}(S),\right.
\end{aligned}
$$

as $D\left(D_{a, b} c\right)=\left[D, D_{a, b}\right] c+D_{a, b}(D c)$. Thus, $D \in \mathscr{M}_{\varphi}$ if and only if $\varphi(D a+\mathscr{I}(S))=0$ for all $a \in S$.

The second assertion of the lemma follows from $[\mathrm{BeM}]$ since $\mathscr{Z}\left(\mathrm{psl}_{3}(S)\right)=$ (0). 
We are now going to construct some Lie algebras which will turn out to give us all of the quasi-simple Lie algebras which we consider. Our classification will be accomplished in the next section. Here we just want to construct the algebras and show they have symmetric non-degenerate invariant bilinear forms.

To begin we take $S=\mathfrak{A}$, the alternative torus. Recalling $\varepsilon: \mathfrak{A} \rightarrow k$ from Definition 1.51 we find that $\varepsilon$ vanishes on $\mathscr{I}(\mathfrak{A})$ and hence induces an element in $(\mathfrak{A} / \mathscr{I}(\mathfrak{A}))^{*}$ which we also denote by $\varepsilon$. We can write $\varepsilon(x)$ for $\varepsilon(x+\mathscr{I}(\mathfrak{A}))$ as $\varepsilon(\mathscr{I}(\mathfrak{A}))=0$. We work with the Lie algebra $\operatorname{ps}_{\mathbf{l}}(\mathfrak{A})=\mathfrak{g}(\mathfrak{A},(0))=\mathfrak{g}$. Now,

$$
\mathfrak{g}=H C_{1}(\mathfrak{A}) \oplus\left(\bigoplus_{\mathfrak{a} \in \mathbb{Z}^{\nu} \backslash \Gamma}\langle\mathfrak{A}, \mathfrak{A}\rangle_{\mathbf{a}}\right) \oplus\left(s l_{3}(k) \otimes \mathfrak{A}\right),
$$

so if $\phi: \mathfrak{g} \rightarrow p s l_{\mathfrak{3}}(\mathfrak{A})$ is the natural homomorphism with kernel $H C_{1}(\mathfrak{A})$ then we know $\phi$ is one-to-one on the subspace $\mathscr{S}=\left(\bigoplus_{\mathfrak{a} \in \mathbb{Z}^{\nu} \backslash \Gamma}\langle\mathfrak{A}, \mathfrak{A}\rangle_{\mathfrak{a}}\right) \oplus\left(s l_{3}(k) \otimes \mathfrak{A}\right)$. Moreover, by Remark 2.27 the form $F=F_{\varepsilon}$ on $\operatorname{psl}_{3}(\mathfrak{A})$ satisfies

$$
\begin{aligned}
& F(x \otimes a, y \otimes b)=\operatorname{Tr}(x y) \varepsilon(a b), \\
& F\left(D_{a, b}, x \otimes c\right)=F\left(x \otimes c, D_{a, b}\right)=0, \text { and } \\
& F\left(D_{a, b}, D_{c, d}\right)=3 \varepsilon\left(\left(D_{a, b} c\right) d\right),
\end{aligned}
$$

for $a, b, c, d \in \mathfrak{A}, x, y \in s l_{3}(k)$.

It is clear that $F$ is non-degenerate on the subspace $s l_{3}(k) \otimes \mathfrak{A} \subseteq p s l_{3}(\mathfrak{A})$. If $D \in \operatorname{Der}_{K}(\mathfrak{A})=\operatorname{Inn}(\mathfrak{A})$ with $F(D, l)=0$ for all $l \in p s l_{3}(\mathfrak{A})$ then for all $a, b \in \mathfrak{A}$ we have

$$
\begin{aligned}
0 & =F\left(D,\left[E_{12} \otimes a, E_{21} \otimes b\right]\right)=F\left(\left[D, E_{12} \otimes a\right], E_{21} \otimes b\right) \\
& =F\left(E_{12} \otimes D a, E_{21} \otimes b\right)=\varepsilon((D a) b) .
\end{aligned}
$$

This clearly implies that $D=0$. Thus, we have

$$
F \text { is non-degenerate on } \operatorname{psl}_{3}(\mathfrak{A}) \text {. }
$$

Thus, since the center of $p \widehat{s l_{3}(\mathfrak{A})}$ is $H C_{1}(\mathfrak{A})$ we get the lift of $F$ to $p \widehat{s l_{3}(\mathfrak{A})}$ has radical equal to $H C_{1}(\mathfrak{A})$. We again denote this lift by $F$. Therefore, on $\mathfrak{g}=p \widehat{s l_{3}(\mathfrak{A})}$ we have $F$ satisfies

$$
\begin{aligned}
& F(x \otimes a, y \otimes b)=\operatorname{Tr}(x y) \varepsilon(a b), \\
& F(\langle a, b\rangle, x \otimes c)=F(x \otimes c,\langle a, b\rangle)=0, \text { and } \\
& F(\langle a, b\rangle,\langle c, d\rangle)=3 \varepsilon\left(\left(D_{a, b} c\right) d\right),
\end{aligned}
$$

for all $a, b, c, d \in \mathfrak{A}, x, y \in s l_{3}(k)$.

As in (2.32) we have $\tilde{\mathscr{M}}_{\varepsilon}=\left\{D \in \operatorname{Der}_{k}(\mathfrak{A}) \mid \varepsilon(D a)=0\right.$ for all $\left.a \in \mathfrak{A}\right\}$. Also, we know $\operatorname{Der}_{k}(\mathfrak{A})=\operatorname{Inn}(\mathfrak{A})+\operatorname{Der}_{k}(K)$ and since our form $F$ is invariant we have $\operatorname{Inn}(\mathfrak{A}) \subseteq \tilde{\mathscr{M}}_{\varepsilon}$. Let

$$
\tilde{\mathscr{D}}=\left\{D \in \operatorname{Der}_{k}(K) \subseteq \operatorname{Der}_{k}(\mathfrak{A}) \subseteq \operatorname{Der}_{k}\left(p \widehat{l_{3}(\mathfrak{A})}\right) \mid D \in \tilde{\mathscr{M}}_{\varepsilon}\right\}
$$

Recalling from Theorem 1.40 that $\operatorname{Der}_{k}(K)=\bigoplus_{\mathbf{a} \in \Gamma} \mathscr{D}_{\mathbf{a}}$ where $\mathscr{D}_{\mathbf{a}}=\left\{\Gamma_{\mathbf{a}, \alpha} \mid \alpha \in\right.$ $\left.k^{\nu}\right\}$ we compute $\Gamma_{\mathbf{a}, \alpha}\left(\left(g^{\mathbf{a}}\right)^{-1}\right)=-(\alpha \mid \mathbf{a}) \cdot 1$ by $(1.31)$ so find that $\varepsilon\left(\Gamma_{\mathbf{a}, \alpha}\left(\left(g^{\mathbf{a}}\right)^{-1}\right)\right)$ $=0$ iff $(\alpha \mid \mathbf{a})=0$. Letting

$$
\tilde{\mathscr{D}}_{\mathbf{a}}=\left\{\Gamma_{\mathbf{a}, \alpha} \mid \alpha \in k^{\nu},(\alpha \mid \mathbf{a})=0\right\},
$$


we find that $\mathscr{D}_{0}=\tilde{\mathscr{D}}_{0}$ and that $\tilde{\mathscr{D}}=\bigoplus_{\mathrm{a} \in \Gamma} \tilde{\mathscr{D}}_{\mathbf{a}}$ so that

$$
\tilde{\mathscr{M}}_{\varepsilon}=\operatorname{Inn}(\mathfrak{A})+\tilde{\mathscr{D}} \text {. }
$$

Now (1.32) implies that $\tilde{\mathscr{D}}$ as well as $\bigoplus_{\mathrm{a} \in \Gamma \backslash\{0\}} \tilde{\mathscr{D}}_{\mathbf{a}}$ are subalgebras of $\operatorname{Der}_{k}(K)$ and that $\tilde{\mathscr{D}}$ consists of the skew-symmetric derivations from Lemma 1.57(a). We have

$$
\operatorname{dim}_{k} \tilde{\mathscr{D}}_{\mathbf{a}}= \begin{cases}\nu & \text { if } \mathbf{a}=0, \\ \nu-1 & \text { if } \mathbf{a} \in \Gamma \backslash\{0\}\end{cases}
$$

By Theorem 1.64

$$
\operatorname{dim}_{k} \tilde{\mathscr{D}}_{\mathbf{a}}=\operatorname{dim}_{k} H C_{1}(\mathfrak{A})_{\mathbf{a}}, \text { for all } \mathbf{a} \in \Gamma .
$$

We now take the semi-direct product of the Lie algebra $p s \widehat{l_{3}(\mathfrak{A})}$ and the algebra $\tilde{\mathscr{D}}$ of derivations of this algebra and from the Lie algebra which we denote $L_{\max }$. Thus,

$$
L_{\max }=p s \widehat{l_{3}(\mathfrak{A})} \oplus \tilde{\mathscr{D}}=\langle\mathfrak{A}, \mathfrak{A}\rangle \oplus\left(s l_{3}(k) \otimes \mathfrak{A}\right) \oplus \tilde{\mathscr{D}} .
$$

For convenience of the reader we recall the multiplication in $L_{\max }$.

$$
\begin{aligned}
{[x} & \left.\otimes g^{\mathbf{a}}, y \otimes g^{\mathbf{b}}\right]=\frac{1}{3} \operatorname{Tr}(x y)\left\langle g^{\mathbf{a}}, g^{\mathbf{b}}\right\rangle+[x, y] \otimes \frac{g^{\mathbf{a}} g^{\mathbf{b}}+g^{\mathbf{b}} g^{\mathbf{a}}}{2} \\
& +\left(x y+y x-\frac{2}{3} \operatorname{Tr}(x y) I_{3}\right) \otimes \frac{g^{\mathbf{a}} g^{\mathbf{b}}-g^{\mathbf{b}} g^{\mathbf{a}}}{2},
\end{aligned}
$$

(2.51) $\left[\Gamma_{\mathbf{a}, \alpha},\left\langle g^{\mathbf{b}}, g^{\mathbf{c}}\right\rangle\right]=-\left[\left\langle g^{\mathbf{b}}, g^{\mathbf{c}}\right\rangle, \Gamma_{\mathbf{a}, \alpha}\right]=\left\langle\Gamma_{\mathbf{a}, \alpha}\left(g^{\mathbf{b}}\right), g^{\mathbf{c}}\right\rangle+\left\langle g^{\mathbf{b}}, \Gamma_{\mathbf{a}, \alpha}\left(g^{\mathbf{c}}\right)\right\rangle$, for all $x, y \in s_{3}(k), \mathbf{a}, \mathbf{b}, \mathbf{c}, \mathbf{d} \in \mathbb{Z}^{\nu}$ and $\alpha, \beta \in k^{\nu}$ with $(\mathbf{a} \mid \alpha)=(\mathbf{b} \mid \beta)=0$.

We wish to extend the form $F$ on $p \widehat{s l_{3}(\mathfrak{A})}$ to the algebra $L_{\max }$ in such a way that we get a form $F$ on $L_{\max }$ which is a non-degenerate invariant symmetric bilinear form. For this write $\mathfrak{g}=p \widehat{s \bar{l}_{3}(\mathfrak{A})}$ and let $\mathfrak{g}_{0}$ be the subspace of $\mathfrak{g}$ given by saying $\mathfrak{g}_{0}=\left(\oplus_{\mathbf{a} \in \mathbb{Z}^{\nu} \backslash \Gamma}\langle\mathfrak{A}, \mathfrak{A}\rangle\right) \oplus\left(s l_{3}(k) \otimes \mathfrak{A}\right)$. Then $F$ on $\mathfrak{g}$ has radical $H C_{1}(\mathfrak{A})$ and is non-degenerate on $\mathfrak{g}_{0}$. We have $L_{\max }=H C_{1}(\mathfrak{A}) \oplus \mathfrak{g}_{0} \oplus \tilde{\mathscr{D}}$. Notice that (2.44) suggests we can extend $F$ by pairing $H C_{1}(\mathfrak{A})$ together with $\tilde{\mathscr{D}}$ in a non-degenerate fashion. We will require that the extension satisfies

$$
\begin{aligned}
& F(\tilde{\mathscr{D}}, \tilde{\mathscr{D}})=(0)=F\left(\tilde{\mathscr{D}}, \mathfrak{g}_{0}\right), \text { and } \\
& F\left(\tilde{\mathscr{D}}_{\mathbf{a}}, H C_{1}(\mathfrak{A})_{\mathbf{b}}\right)=0 \text { unless } \mathbf{a}+\mathbf{b}=0 \text { for } \mathbf{a}, \mathbf{b} \in \mathbb{Z}^{\nu} .
\end{aligned}
$$

Recalling that Lemma 1.56 tells us $H C_{1}(\mathfrak{A})_{\mathbf{a}}$ is spanned by the elements $\left\langle g^{\mathbf{a}} g_{i}^{-1}, g_{i}\right\rangle, \quad 1 \leq i \leq \nu$ when $\mathbf{a} \in \mathbb{Z}^{\nu}$. Thus, if $F$ exists it must satisfy $F\left(\Gamma_{-\mathbf{a}, \alpha},\left[E_{12} \otimes g^{\mathbf{a}} g_{i}^{-1}, E_{21} \otimes g_{i}\right]\right)=F\left(E_{12} \otimes \Gamma_{-\mathbf{a}, \alpha}\left(g^{\mathbf{a}} g_{i}^{-1}\right), E_{21} \otimes g_{i}\right)=-\left(\alpha, \epsilon_{i}\right)$. 
On the other hand, assuming (2.52) and (2.53) and using (2.46) we have

$$
F\left(\Gamma_{-\mathbf{a}, \alpha},\left[E_{12} \otimes g^{\mathbf{a}} g_{i}^{-1}, E_{21} \otimes g_{i}\right]\right)=\frac{1}{3} F\left(\Gamma_{-\mathbf{a}, \alpha},\left\langle g_{a} g_{i}^{-1}, g_{i}\right\rangle\right) .
$$

This leads to

$$
F\left(\Gamma_{-\mathbf{a}, \alpha},\left\langle g^{\mathbf{a}} g_{i}^{-1}, g_{i}\right\rangle\right)=-3\left(\alpha \mid \epsilon_{i}\right) \cdot 1, \quad 1 \leq i \leq \nu, \mathbf{a} \in \mathbb{Z}^{\nu} .
$$

Recalling the notation of Lemma 1.50 we would then have

$$
F\left(\Gamma_{-\mathbf{a}, \alpha}, g^{\mathbf{a}} \omega_{\beta}\right)=F\left(g^{\mathbf{a}} \omega_{\beta}, \Gamma_{-\mathbf{a}, \alpha}\right)=-3(\alpha \mid \beta),
$$

where $\mathbf{a} \in \mathbb{Z}^{\nu}, \alpha, \beta \in k^{\nu}$ and $(\mathbf{a} \mid \alpha)=0$. Moreover, we know from the proof of Lemma 1.57 that $g^{\mathbf{a}} \omega_{\beta}=0$ if and only if $\beta \in k \mathbf{a}$ so the above formulas can be used to give a well-defined extension to a symmetric bilinear form on $L_{\max }$, which is clearly non-degenerate. We check this form is invariant in the next lemma.

Lemma 2.55. (1) Let $\mathbf{a} \in \Gamma, \alpha \in k^{\nu}$ with $(\mathbf{a} \mid \alpha)=0$ and let $\mathbf{b}, \mathbf{c} \in \mathbb{Z}^{\nu}$. Then $F\left(\Gamma_{-\mathbf{a}, \alpha},\left\langle g^{\mathbf{b}}, g^{\mathbf{c}}\right\rangle\right)=3 \varepsilon\left(\Gamma_{-\mathbf{a}, \alpha}\left(g^{\mathbf{b}}\right) g^{\mathbf{c}}\right)$.

(2) The form $F$ is an invariant non-degenerate symmetric bilinear form on $L_{\max }$.

Proof. If $\mathbf{b}+\mathbf{c} \neq \mathbf{a}$ then both sides in (1) are zero so assume $\mathbf{b}+\mathbf{c}=\mathbf{a} \in \Gamma$. Thus $\mathbf{b}=\mathbf{a}-\mathbf{c}$ and hence $\left\langle g^{\mathbf{b}}, g^{\mathbf{c}}\right\rangle=\left\langle g^{\mathbf{a}-\mathbf{c}}, g^{\mathbf{c}}\right\rangle=\left\langle g^{\mathbf{a}} g^{-\mathbf{c}}, g^{\mathbf{c}}\right\rangle$ because $\mathbf{a} \in \Gamma$ so $g^{\mathbf{a}} \in K$. Let $g^{-\mathbf{c}}=s\left(g^{\mathbf{c}}\right)^{-1}$ where $s \in k, s \neq 0$. Then $\left\langle g^{\mathbf{b}}, g^{\mathbf{c}}\right\rangle=$ $s\left\langle g^{\mathbf{a}}\left(g^{\mathbf{c}}\right)^{-1}, g^{\mathbf{c}}\right\rangle=s \sum_{i=1}^{\nu} c_{i}\left\langle g^{\mathbf{a}} g_{i}^{-1}, g_{i}\right\rangle$ by Lemma 1.50. Thus,

$$
F\left(\Gamma_{-\mathbf{a}, \alpha},\left\langle g^{\mathbf{b}}, g^{\mathbf{c}}\right\rangle\right)=s \sum_{i=1}^{\nu} c_{i} F\left(\Gamma_{-\mathbf{a}, \alpha},\left\langle g^{\mathbf{a}} g_{i}^{-1}, g_{i}\right\rangle\right)=-3 s(\mathbf{c} \mid \alpha)=3 s(\mathbf{b} \mid \alpha)
$$

because $\mathbf{b}=\mathbf{a}-\mathbf{c}$ and $(\mathbf{a} \mid \alpha)=0$. Also, we have

$$
3 \varepsilon\left(\Gamma_{-\mathbf{a}, \alpha}\left(g^{\mathbf{b}}\right) g^{\mathbf{c}}\right)=3(\alpha \mid \mathbf{b}) \varepsilon\left(\left(g^{-\mathbf{a}} g^{\mathbf{b}}\right) g^{\mathbf{c}}\right)=3(\alpha \mid \mathbf{b}) s,
$$

and this proves (1).

As for invariance we know that $F$ is already invariant on $\mathfrak{g}$ and if $D_{1}, D_{2}$, $D_{3} \in \tilde{\mathscr{D}}$ then both of $F\left(\left[D_{1}, D_{2}\right], D_{3}\right)$ and $F\left(D_{1},\left[D_{2}, D_{3}\right]\right)$ are zero. Thus, if $g, g_{1}, g_{2} \in \mathfrak{g}$ and $D, D_{1}, D_{2} \in \mathscr{D}$ we need only check

$$
\begin{aligned}
& F\left(D,\left[g_{1}, g_{2}\right]\right)=F\left(\left[D, g_{1}\right], g_{2}\right), \\
& F\left(D_{1},\left[D_{2}, g\right]\right)=F\left(\left[D_{1}, D_{2}\right], g\right), \text { and } \\
& F\left(\left[D_{1}, g\right], D_{2}\right)=F\left(D_{1},\left[g, D_{2}\right]\right),
\end{aligned}
$$

noting that we already know $F\left(\left[D, g_{1}\right], g_{2}\right)=-F\left(g_{1},\left[D, g_{2}\right]\right)$ since $\tilde{\mathscr{D}}$ consists of skew-symmetric derivations of $\mathfrak{g}$ with respect to $F$.

We do these in order. If one of $g_{1}$ or $g_{2}$ is in $H C_{1}(\mathfrak{A})$ then both sides of $(\mathrm{A})$ are zero. Thus, we can assume $g_{1}, g_{2} \in \mathfrak{g}_{0}$. If one of $g_{1}$ or $g_{2}$ is in $\langle\mathfrak{A}, \mathfrak{A}\rangle_{\mathbf{a}}$ for $\mathbf{a} \notin \Gamma$ and the other is in $s l_{3}(k) \otimes \mathfrak{A}$ then again both sides are zero. Thus, we can assume that either $g_{1}, g_{2} \in s l_{3}(k) \otimes \mathfrak{A}$ or both are in $\bigoplus_{\mathbf{a} \in \mathbb{Z}^{\nu} \backslash \Gamma}\langle\mathfrak{A}, \mathfrak{A}\rangle_{\mathbf{a}}$. For the first of these we take $D=\Gamma_{\mathbf{a}, \alpha}, g_{1}=x \otimes g^{\mathbf{b}}, g_{2}=y \otimes g^{\mathbf{c}}$ when $\mathbf{a} \in \Gamma, \alpha \in k^{\nu},(\mathbf{a} \mid \alpha)=0, x, y \in \operatorname{sl}_{3}(k)$ and $\mathbf{b}, \mathbf{c} \in \mathbb{Z}^{\nu}$. We may as well assume 
that $\mathbf{a}+\mathbf{b}+\mathbf{c}=0$ for otherwise both sides equal zero. Now the left-hand side of $(\mathrm{A})$ is, by Lemma 2.55(1) and Definition (1.31),

$$
\begin{aligned}
F\left(\Gamma_{\mathbf{a}, \alpha},\left[x \otimes g^{\mathbf{b}}, y \otimes g^{\mathbf{c}}\right]\right) & =F\left(\Gamma_{\mathbf{a}, \alpha}, \frac{1}{3} \operatorname{Tr}(x y)\left\langle g^{\mathbf{b}}, g^{\mathbf{c}}\right\rangle\right) \\
& =(\alpha \mid \mathbf{b}) \operatorname{Tr}(x y) \varepsilon\left(\left(g^{\mathbf{a}} g^{\mathbf{b}}\right) g^{\mathbf{c}}\right) .
\end{aligned}
$$

Next we have $\left[\Gamma_{\mathbf{a}, \alpha}, x \otimes g^{\mathbf{b}}\right]=(\alpha \mid \mathbf{b}) x \otimes g^{\mathbf{a}} g^{\mathbf{b}}$ so the right-hand side of $(\mathrm{A})$ is

$$
(\alpha \mid \mathbf{b}) F\left(x \otimes g^{\mathbf{a}} g^{\mathbf{b}}, y \otimes g^{\mathbf{c}}\right)=(\alpha \mid \mathbf{b}) \operatorname{Tr}(x y) \varepsilon\left(\left(g^{\mathbf{a}} g^{\mathbf{b}}\right) g^{\mathbf{c}}\right)
$$

as desired.

Now assume both $g_{1}, g_{2}$ are in $\bigoplus_{\mathbf{a} \in \mathbb{Z}^{\nu} \backslash \Gamma}\langle\mathfrak{A}, \mathfrak{A}\rangle_{\mathbf{a}}$ and say $g_{1}=\left\langle g^{\mathbf{b}}, g^{\mathbf{c}}\right\rangle, g_{2}=$ $\left\langle g^{\mathbf{d}}, g^{\mathbf{e}}\right\rangle$ and $D \in \tilde{\mathscr{D}}$. We must show

$$
F\left(D,\left[\left\langle g^{\mathbf{b}}, g^{\mathbf{c}}\right\rangle,\left\langle g^{\mathbf{d}}, g^{\mathbf{e}}\right\rangle\right]\right)=F\left(\left[D,\left\langle g^{\mathbf{b}}, g^{\mathbf{c}}\right\rangle\right],\left\langle g^{\mathbf{d}}, g^{\mathbf{e}}\right\rangle\right) \text {. }
$$

The left-hand side of this is, by Lemma 2.55(1),

$$
\begin{aligned}
F(D, & \left.\left\langle D_{g^{\mathbf{b}}, g^{\mathbf{c}}}\left(g^{\mathbf{d}}\right), g^{\mathbf{e}}\right\rangle+\left\langle g^{\mathbf{d}}, D_{g^{\mathbf{b}}, g^{\mathbf{c}}}\left(g^{\mathbf{e}}\right)\right\rangle\right) \\
& =3 \varepsilon\left(\left(D\left(D_{g^{\mathbf{b}}, g^{\mathbf{c}}}\left(g^{\mathbf{d}}\right)\right)\right) g^{\mathbf{e}}+\left(D\left(g^{\mathbf{d}}\right)\right)\left(D_{g^{\mathbf{b}}, g^{\mathbf{c}}}\left(g^{\mathbf{e}}\right)\right)\right) .
\end{aligned}
$$

The right-hand side is

$$
\begin{aligned}
& F\left(\left\langle D\left(g^{\mathbf{b}}\right), g^{\mathbf{c}}\right\rangle+\left\langle g^{\mathbf{b}}, D\left(g^{\mathbf{c}}\right)\right\rangle,\left\langle g^{\mathbf{d}}, g^{\mathbf{e}}\right\rangle\right) \\
& \quad=3 \varepsilon\left(\left(D_{D\left(g^{\mathbf{b}}\right), g^{\mathbf{c}}}\left(g^{\mathbf{d}}\right)\right) g^{\mathbf{e}}+\left(D_{g^{\mathbf{b}}, D\left(g^{\mathbf{c}}\right)}\left(g^{\mathbf{d}}\right)\right) g^{\mathbf{e}}\right) .
\end{aligned}
$$

Now $D\left(D_{a, b}(c)\right)=D_{D a, b}(c)+D_{a, D b}(c)+D_{a, b}(D c)$ so the left-hand side becomes

$$
3 \varepsilon\left(D_{D\left(g^{\mathbf{b}}\right), g^{\mathbf{c}}}\left(g^{\mathbf{d}}\right) g^{\mathbf{e}}+D_{g^{\mathbf{b}}, D\left(g^{\mathbf{c}}\right)}\left(g^{\mathbf{d}}\right) g^{\mathbf{e}}+D_{g^{\mathbf{b}}, g^{\mathbf{c}}}\left(D g^{\mathbf{d}}\right) g^{\mathbf{e}}+\left(D\left(g^{\mathbf{d}}\right)\right) D_{g^{\mathbf{b}}, g^{\mathbf{c}}}\left(g^{\mathbf{e}}\right)\right) \text {. }
$$

Taking the difference of the two sides gives

$$
3 \varepsilon\left(D_{g^{\mathrm{b}}, g^{\mathrm{c}}}\left(D\left(g^{\mathbf{d}}\right)\right) g^{\mathbf{e}}+D\left(g^{\mathbf{d}}\right) D_{g^{\mathrm{b}}, g^{\mathrm{c}}}\left(g^{u} e\right)\right)=3 \varepsilon\left(D_{g^{\mathrm{b}}, g^{\mathrm{c}}}\left(D\left(g^{\mathbf{d}}\right) g^{\mathbf{e}}\right)\right)=0
$$

because of (2.31) and the fact that $\operatorname{Inn}(\mathfrak{A}) \subseteq \tilde{\mathscr{M}}_{\varepsilon}$. This takes care of case (A).

To check (B) note that both sides are zero if $g \in \mathfrak{g}_{0}$ so we assume $g \in$ $H C_{1}(\mathfrak{A})$. Take $D_{1}=\Gamma_{\mathbf{a}, \alpha}, D_{2}=\Gamma_{\mathbf{b}, \beta}, g=g^{\mathbf{c}} \omega_{\gamma}$ where $\mathbf{a}, \mathbf{b}, \mathbf{c} \in \Gamma,(\mathbf{a} \mid \alpha)=$ $(\mathbf{b} \mid \beta)=0$ and $\alpha, \beta, \gamma \in k^{\nu}$. By (1.32) we have $\left[\Gamma_{\mathbf{a}, \alpha}, \Gamma_{\mathbf{b}, \beta}\right]=\Gamma_{\mathbf{a}+\mathbf{b},(\alpha \mid \mathbf{b}) \beta-(\beta \mid \mathbf{a}) \alpha}$ so noting both sides of (B) are zero unless $\mathbf{a}+\mathbf{b}+\mathbf{c}=0$ we have from (2.54) that

$$
F\left(\left[\Gamma_{\mathbf{a}, \alpha}, \Gamma_{\mathbf{b}, \beta}\right], g^{\mathbf{c}} \omega_{\gamma}\right)=-3((\alpha \mid \mathbf{b}) \beta-(\beta \mid \mathbf{a}) \alpha \mid \gamma) .
$$

By Lemma 1.50 we have $\Gamma_{\mathbf{b}, \beta}\left(g^{\mathbf{c}} \omega_{\gamma}\right)=g^{\mathbf{b}+\mathbf{c}} \omega_{(\beta \mid \mathbf{c})+(\beta \mid \gamma) \mathbf{b}}$, so

$$
\begin{gathered}
F\left(\Gamma_{\mathbf{a}, \alpha},\left[\Gamma_{\mathbf{b}, \beta}, g^{\mathbf{c}} \omega_{\gamma}\right]\right)=-3(\alpha \mid(\beta \mid \mathbf{c}) \gamma+(\beta \mid \gamma) \mathbf{b}) \\
=-3(\alpha \mid \gamma)(\beta \mid \mathbf{c})-3(\beta \mid \gamma)(\alpha \mid \mathbf{b}) .
\end{gathered}
$$

Now as $\mathbf{a}+\mathbf{b}+\mathbf{c}=0$ and $(\beta \mid \mathbf{b})=0$ we see that $(\beta \mid \mathbf{c})=-(\beta \mid \mathbf{a})$ and so both sides are equal. This takes care of $(B)$, and case $(C)$ is checked in exactly the same way.

It is worth noting that Lemma 2.55 (1) can be written as

$$
F(D,\langle x, y\rangle)=3 \varepsilon(D(x) y),
$$

for all $D \in \tilde{\mathscr{D}}$ and $x, y \in \mathfrak{A}$. 
We now introduce the class of Lie algebras which will be the objects of study in the next section. Let $\mathscr{D}^{\prime}$ be any subalgebra of $\tilde{\mathscr{D}}$ which contains $\mathscr{D}_{0}$ and note that since $\mathscr{D}_{0}$ has as basis the degree derivations $d_{1}, \ldots, d_{\nu}$ then $\mathscr{D}^{\prime}$ is a homogeneous subalgebra of $\tilde{\mathscr{D}}$ in the $\Gamma$-gradation. We let $\mathscr{E}\left(\mathscr{D}^{\prime}\right)$ be the orthogonal complement of $\mathscr{D}^{\prime}$ in $H C_{1}(\mathfrak{A})$. That is

$$
\mathscr{E}\left(\mathscr{D}^{\prime}\right)=\mathscr{D}^{\prime \perp} \cap H C_{1}(\mathfrak{A}),
$$

and this subspace of $H C_{1}(\mathfrak{A})$ is also homogeneous in the $\Gamma$-gradation since $F\left(\tilde{\mathscr{D}}_{\mathbf{a}}, H C_{1}(\mathfrak{A})_{\mathbf{b}}\right)=0$ unless $\mathbf{a}+\mathbf{b}=0$. Since $F$ is non-degenerate on $\tilde{\mathscr{D}} \times$ $H C_{1}(\mathfrak{A})$ we get an induced non-degenerate form on $\mathscr{D}^{\prime} \times H C_{1}(\mathfrak{A}) / \mathscr{E}\left(\mathscr{D}^{\prime}\right)$. Moreover if $x \in \mathscr{E}\left(\mathscr{D}^{\prime}\right), E_{1}, E_{2} \in \mathscr{D}^{\prime}$, then $F\left(E_{1},\left[E_{2}, x\right]\right)=F\left(\left[E_{1}, E_{2}\right], x\right)$ $=0$ as $\mathscr{D}^{\prime}$ is a subalgebra. Thus, $\left[\mathscr{D}^{\prime}, \mathscr{E}\left(\mathscr{D}^{\prime}\right)\right] \subseteq \mathscr{E}\left(D^{\prime}\right)$. We then form the semidirect product Lie algebra $p s \widehat{l_{3}(\mathfrak{A})} \oplus \mathscr{D}^{\prime} \subseteq L_{\max }$ and note that $\mathscr{E}\left(\mathscr{D}^{\prime}\right)$ is then an ideal of this Lie algebra. Factoring and doing the obvious identifications we obtain the Lie algebra

$$
H C_{1}(\mathfrak{A}) / \mathscr{E}\left(\mathscr{D}^{\prime}\right) \oplus\left(\bigoplus_{\mathfrak{a} \in \mathbb{Z}^{\nu} \backslash \Gamma}\langle\mathfrak{A}, \mathfrak{A}\rangle_{\mathbf{a}}\right) \oplus\left(s l_{3}(k) \otimes \mathfrak{A}\right) \oplus \mathscr{D}^{\prime}=\mathfrak{g}\left(\mathfrak{A}, \mathscr{E}\left(\mathscr{D}^{\prime}\right)\right) \oplus \mathscr{D}^{\prime}
$$

We name this algebra in the following definition.

Definition 2.58. The Lie algebra $L\left(\mathscr{D}^{\prime}\right)$ is defined as $L\left(\mathscr{D}^{\prime}\right)=\mathfrak{g}\left(\mathfrak{A}, \mathscr{E}\left(\mathscr{D}^{\prime}\right)\right) \oplus$ $\mathscr{D}^{\prime}$. The special case when $\mathscr{D}^{\prime}=\mathscr{D}_{0}$ has $\mathscr{E}\left(\mathscr{D}^{\prime}\right)=\bigoplus_{\mathrm{a} \in \Gamma \backslash\{0\}} H C_{1}(\mathfrak{A})_{\mathbf{a}}$ so that $H C_{1}(\mathfrak{A}) / \mathscr{E}\left(\mathscr{D}^{\prime}\right) \cong H C_{1}(\mathfrak{A})_{0}$ (and we usually identify these spaces) and we denote the resulting algebra by $L_{\text {min }}$. Thus $L_{\text {min }}=\mathfrak{g}\left(\mathfrak{A}, \mathscr{E}\left(\mathscr{D}_{0}\right)\right) \oplus \mathscr{D}_{0}$, and $L_{\max }=L(\tilde{\mathscr{D}})$.

It is clear that the restriction of our form $F$ on $L_{\max }$ to the subalgebra ps $\widehat{l_{3}(\mathfrak{A})} \oplus \mathscr{D}^{\prime}$ has radical equal to $\mathscr{E}\left(\mathscr{D}^{\prime}\right)$, so the quotient algebra inherits a non-degenerate form which we also denote by $F$.

Corollary 2.59. The form on $L_{\max }$ gives rise to an invariant symmetric nondegenerate bilinear form on $L\left(\mathscr{D}^{\prime}\right)$ for any subalgebra $\mathscr{D}^{\prime}$ of $\tilde{\mathscr{D}}$ which contains $\mathscr{D}_{0}$.

There is one further refinement which we need to make to our construction. As in $[B G K]$ we can adjust the algebras $L\left(\mathscr{D}^{\prime}\right)$ by a homogeneous 2-cocycle of invariant type. To present the definition we need to develop a little more notation as follows. If $\mathscr{D}^{\prime}$ is a subalgebra of $\tilde{\mathscr{D}}$ containing $\mathscr{D}_{0}$ we have

$$
\begin{aligned}
& \mathscr{D}^{\prime}=\mathscr{D}_{0} \oplus_{\mathbf{a} \in \Gamma \backslash\{0\}} \mathscr{D}_{\mathbf{a}}^{\prime}, \text { where } \\
& \mathscr{D}_{\mathbf{a}}^{\prime}=\mathscr{D}^{\prime} \cap \tilde{D}_{\mathbf{a}} \text { for } \mathbf{a} \in \Gamma, \text { and we let } \\
& \mathscr{D}^{\prime \prime}=\bigoplus_{\mathbf{a} \in \Gamma \backslash\{0\}} \mathscr{D}_{\mathbf{a}}^{\prime}, \text { so that } \mathscr{D}^{\prime}=\mathscr{D}_{0} \oplus \mathscr{D}^{\prime \prime} .
\end{aligned}
$$

The center of $\mathfrak{g}\left(\mathfrak{A}, \mathscr{E}\left(\mathscr{D}^{\prime}\right)\right)$ is $H C_{1}(\mathfrak{A}) / \mathscr{E}\left(\mathscr{D}^{\prime}\right)$ and this is homogeneous in the $\Gamma$-gradation. We write this as $\mathscr{Z}$ (or $\mathscr{Z}\left(\mathscr{D}^{\prime}\right)$ if we need to make reference to 
$\mathscr{D}^{\prime}$. Thus,

$$
\begin{aligned}
& \mathscr{Z}=H C_{1}(\mathfrak{A}) / \mathscr{E}\left(\mathscr{D}^{\prime}\right) \text {, and } \\
& \mathscr{Z}=\bigoplus_{\mathbf{a} \in \Gamma} \mathscr{Z}_{\mathbf{a}}, \text { where we know } \\
& \operatorname{dim} \mathscr{Z}_{\mathbf{a}}=\operatorname{dim} \mathscr{D}_{\mathbf{a}}^{\prime}, \text { for all } \mathbf{a} \in \Gamma .
\end{aligned}
$$

Definition 2.66. Let the notation be as above. A mapping $\tau: \mathscr{D}^{\prime \prime} \times \mathscr{D}^{\prime \prime} \rightarrow$ $\mathscr{Z}\left(\mathscr{D}^{\prime}\right)$ is a homogeneous 2-cocycle of invariant type if and only if

$$
\begin{aligned}
& \tau\left(\mathscr{D}_{\mathbf{a}}^{\prime}, \mathscr{D}_{\mathbf{b}}^{\prime}\right) \subset \mathscr{Z}_{\mathbf{a}+\mathbf{b}}, \text { for } \mathbf{a}, \mathbf{b} \in \Gamma \backslash\{0\}, \\
& \tau(x, y)=-\tau(y, x), \\
& \tau([x, y], z)+\tau([y, z], x)+\tau([z, x], y) \\
& \quad+[\tau(x, y), z]+[\tau(y, z), x]+[\tau(z, x), y]=0, \text { and } \\
& F(\tau(x, y), z)=F(x, \tau(y, z)) \text { for all } x, y, z \in \mathscr{E}^{\prime} .
\end{aligned}
$$

We can use any cocycle of Definition 2.66 to twist the multiplication in $L\left(\mathscr{D}^{\prime}\right)$ and obtain an algebra $L\left(\mathscr{D}^{\prime}, \tau\right)$. The new multiplication in $L\left(\mathscr{D}^{\prime}, \tau\right)$ is exactly like the old one in $L\left(\mathscr{D}^{\prime}\right)$ except that product of two elements, say $x, y \in \mathscr{D}^{\prime}$, is given by

$$
[x, y]_{\text {new }}=[x, y]_{\text {old }}+\tau(x, y) .
$$

Now (a) of Definition 2.66 will insure that the grading of $L\left(\mathscr{D}^{\prime}\right)$ induces one of $L\left(\mathscr{D}^{\prime}, \tau\right)$ while it is clear that (b) and (c), along with the fact that $\mathfrak{g}\left(\mathfrak{A}, \mathscr{E}\left(\mathscr{D}^{\prime}\right)\right)$ is an ideal of $L\left(\mathscr{D}^{\prime}\right)$, give us that $L\left(\mathscr{D}^{\prime}, \tau\right)$ is a Lie algebra. Finally condition (d) together with the fact that $\mathscr{Z}\left(\mathscr{D}^{\prime}\right)$ is central in $\mathfrak{g}\left(\mathfrak{A}, \mathscr{D}^{\prime}\right) \subseteq L\left(\mathscr{D}^{\prime}\right)$ gives that the form $F$ on $L\left(\mathscr{D}^{\prime}, \tau\right)$ is still a non-degenerate invariant symmetric bilinear form. We record this as follows.

Corollary 2.68. For any subalgebra $\mathscr{D}^{\prime}$ of $\tilde{\mathscr{D}}$ containing $\mathscr{D}_{0}$ and for any homogeneous 2-cocycle $\tau: \mathscr{D}^{\prime \prime} \times \mathscr{D}^{\prime \prime} \rightarrow \mathscr{Z}\left(\mathscr{D}^{\prime}\right)$ which is of invariant type, the Lie algebra $L\left(\mathscr{D}^{\prime}, \tau\right)$ has a non-degenerate invariant symmetric bilinear form obtained from the form on $L\left(\mathscr{D}^{\prime}\right)$.

Remark 2.69. (i) If $\mathscr{D}^{\prime}=\mathscr{D}_{0}$ no cocyles are allowed according to Definition 2.66, so $L\left(\mathscr{D}_{0}, \tau\right)$ will just denote $L\left(\mathscr{D}_{0}\right)=L_{\text {min }}$.

(ii) The zero map of $\mathscr{D}^{\prime \prime} \times \mathscr{D}^{\prime \prime} \rightarrow \mathscr{Z}\left(\mathscr{D}^{\prime}\right)$ is always an invariant homogeneous 2-cocycle and this just yields the algebra $L\left(\mathscr{D}^{\prime}\right)$. That is, $L\left(\mathscr{D}^{\prime}\right)=L\left(\mathscr{D}^{\prime}, 0\right)$.

\section{The Classification of QS algebras of TYPE $A_{2}$}

In this final section we are going to classify the tame elliptic irreducible quasisimple Lie algebras of type $A_{2}$ over the complex field $\mathbb{C}$. We call them QS algebras and it turns out that if the coordinate algebra is an alternative algebra which is not associative then the QS algebra is isomorphic to one of our algebras $L\left(\mathscr{D}^{\prime}, \tau\right)$ of Section 2 . In the case when the coordinates are associative then the coordinate algebra is a quantum torus $\mathbb{C}_{\mathbf{q}}$ for some $\nu \times \nu$ matrix $\mathbf{q}$ as in (1.1), and the QS algebra is just like those considered in [BGK], and so we only deal with the alternative, not the associative, case here. In fact, even in this case the arguments are almost exactly as in [BGK] and hence we will be brief and refer the reader to [BGK] for more details. Indeed the general theory of QS 
algebras as developed in [H-KT] and [BGK] is what we need in this section, so we will only recall the basic definitions and results which we need to establish our main theorem. All our algebras will be over the base field $\mathbb{C}$ of complex numbers.

To begin the definitions we need from [H-KT] and [BGK] we let $\mathscr{L}$ be a Lie algebra and assume that

$$
(\cdot, \cdot): \mathscr{L} \times \mathscr{L} \rightarrow \mathbb{C},
$$

is a non-degenerate invariant symmetric bilinear form and let $\mathscr{H} \subseteq \mathscr{L}$ be a finite dimensional abelian subalgebra such that

$$
\operatorname{ad}_{\mathscr{L}} h \text { is diagonalizable for all } h \in \mathscr{H} \text {. }
$$

We let $\mathscr{L}_{\alpha}=\{x \in \mathscr{L} \mid[h, x]=\alpha(h) x$ for all $h \in \mathscr{H}\}$ for all $\alpha \in \mathscr{H}^{*}$ (the dual space of $\mathscr{H})$, and let $R=\left\{\alpha \in \mathscr{H}^{*} \mid \mathscr{L}_{\alpha} \neq(0)\right\}$. We assume that

$$
\mathscr{L}_{0}=\mathscr{H},
$$

and note that we have the root space decomposition

$$
\mathscr{L}=\bigoplus_{\alpha \in R} \mathscr{L}_{\alpha}
$$

Then one can show that $\left(\mathscr{L}_{\alpha}, \mathscr{L}_{\beta}\right)=(0)$ if $\alpha+\beta \neq 0$ so the form $(\cdot, \cdot)$ is nondegenerate on $\mathscr{H}$ and on $\mathscr{L}_{\alpha}+\mathscr{L}_{-\alpha}$, for all $\alpha \in R$ so that $-R=R$. Moreover, $\left[\mathscr{L}_{\alpha}, \mathscr{L}_{\beta}\right] \subseteq \mathscr{L}_{\alpha+\beta}$ and if $x_{\alpha} \in \mathscr{L}_{\alpha}, x_{-\alpha} \in \mathscr{L}_{-\alpha}$ then letting $h_{\alpha} \in \mathscr{H}$ be given by $\left(h_{\alpha}, h\right)=\alpha(h)$ for all $h \in \mathscr{H}, \alpha \in \mathscr{H}^{*}$ we obtain

$$
\left[x_{\alpha}, x_{-\alpha}\right]=\left(x_{\alpha}, x_{-\alpha}\right) h_{\alpha} \text {. }
$$

One transfers the form $(\cdot, \cdot)$ on $\mathscr{H}$ to $\mathscr{H}^{*}$ by

$$
(\lambda, \mu)=\left(h_{\lambda}, h_{\mu}\right)=\lambda\left(h_{\mu}\right)=\mu\left(h_{\lambda}\right),
$$

for all $\lambda, \mu \in \mathscr{H}^{*}$.

We assume that

$$
R \text { is discrete, and }
$$

Thus, on the real vector space $\mathscr{H}_{R}^{*}=\sum_{\alpha \in R} \mathbb{R} \alpha$ (the real span of the roots), the form takes on real values.

An element $\delta \in \mathscr{H}^{*}$ is isotropic if and only if $(\delta, \delta)=0$ and we assume that

$$
\text { if } \alpha \in R \text { is non-isotropic and } x \in \mathscr{L}_{\alpha} \text {, then } \operatorname{ad}_{\mathscr{L}} x \text { is nilpotent. }
$$

Any Lie algebra $\mathscr{L}$ satisfying (3.1) through (3.9) is said to be a quasi-simple Lie algebra. If $\alpha \in R$ is non-isotropic and lets $\omega_{\alpha} \in G L\left(\mathscr{H}^{*}\right)$ be defined by

$$
\omega_{\alpha}(\beta)=\beta-\frac{2(\beta, \alpha)}{(\alpha, \alpha)} \alpha .
$$

One of the main results on quasi-simple algebras from [H-KT] is the following, also see [BGK]. 
Theorem 3.11. Let $\mathscr{L}$ be a quasi-simple Lie algebra with root system $R$ and let $\alpha \in R$ be non-isotropic. Then

(i) $\operatorname{dim} \mathscr{L}_{\alpha}=1$,

(ii) $\frac{2(\beta, \alpha)}{(\alpha, \alpha)} \in \mathbb{Z}$ (the integers) for all $\beta \in R$,

(iii) $\omega_{\alpha}(\beta) \in R$ for all $\beta \in R$ so $\omega_{\alpha}(R)=R$,

(iv) for $k \in \mathbb{C}$ we have $k \alpha \in R$ if and only if $k= \pm 1$ or $k=0$,

(v) for any $\beta \in R$ there exist nonnegative integers $n_{-}, n_{+}$such that for $n \in \mathbb{C}, \beta+n \alpha \in R$ if and only if $n \in \mathbb{Z}$ and $-n_{-} \leq n \leq n_{+}$, and also $n_{-}-n_{+}=\frac{2(\beta, \alpha)}{(\alpha, \alpha)}$,

(vi) for any isotropic root $\delta \in R$ we have $(\beta, \delta)=0$ for all $\beta \in R$.

(3.12) One says that $\mathscr{L}$ is of elliptic type if the form on $\mathscr{H}_{R}^{*}$ is positive semidefinite.

We always assume $\mathscr{L}$ is elliptic and we let

$$
\Lambda=\{\delta \in R \mid \delta \text { is isotropic }\} \text {. }
$$

We define a map $\mathfrak{l}: \mathscr{H}_{R}^{*} \rightarrow\left(\mathscr{H}_{R}^{*}\right)^{*}$ by $\mathfrak{l}(\lambda)(\mu)=(\lambda, \mu)$ for all $\lambda, \mu \in \mathscr{H}_{R}^{*}$, where $\left(\mathscr{H}_{R}^{*}\right)^{*}$ is the real dual of $\mathscr{H}_{R}^{*}$. Then (vi) of Theorem 3.11 implies that the real span of $\Lambda$ is in the kernel of $\mathfrak{l}$. Using this linear map $\mathfrak{l}$ one transfers the form from $\mathscr{H}_{R}^{*}$ to $\mathfrak{l}\left(\mathscr{H}_{R}^{*}\right)$ and lets $\Delta=\mathfrak{l}(R) \subseteq \mathfrak{l}\left(\mathscr{H}_{R}^{*}\right)$. It is easy to see (as in [H-KT]) the transferred form on $\mathrm{l}\left(\mathscr{H}_{R}^{*}\right)$ is positive definite and $\Delta$ is a finite root system which is possibly non-reduced. One now assumes that the root system of $\mathscr{L}$ is irreducible in the sense that

$\Delta$ is an irreducible finite root system, and

(3.15) if $\delta \in R$ is isotropic there is some non-isotropic root $\alpha \in R$ for which $\alpha+\delta \in R$.

We say $\mathscr{L}$ is of type $X_{l}$ if $\Delta$ is a finite root system of type $X_{l}$ and we now assume $\Delta$ is of type $A_{2}$. Letting $K_{R}$ be the kernel of $\mathfrak{l}$ we define the nullity of $\mathscr{L}$, denoted $\nu$ or $\nu(\mathscr{L})$, by

$$
\nu=\operatorname{dim} K_{R} .
$$

Clearly $\operatorname{dim} \mathscr{H}_{R}^{*}=\nu+2$.

As in Definition 2.6 we let $\Delta$ be the root system of type $A_{2}$. Then as in [H-KT] one has $R$ can be identified with $\Delta \times \Lambda$ so that

$$
R=\Delta \times \Lambda \subseteq \mathscr{H}_{R}^{*} \subseteq \mathscr{H}^{*} \text {. }
$$

Moreover, $\Lambda$ is a lattice in $K_{R}$ of rank $\nu$ so letting $\delta_{1}, \ldots, \delta_{\nu}$ be a basis of $\Lambda$ which is also a basis of $K_{R}$, we have

$$
\Lambda=\mathbb{Z} \delta_{1} \oplus \cdots \oplus \mathbb{Z} \delta_{\nu} .
$$

As in [BGK] there are elements $h_{1}=h_{\alpha_{1}}, h_{2}=h_{\alpha_{2}}, h_{\delta_{1}}, \ldots, h_{\delta_{\nu}}$ corresponding to the roots $\alpha_{1}, \alpha_{2}, \delta_{1}, \ldots, \delta_{\nu}$ and hence there are elements $d_{1}, \ldots, d_{\nu} \in$ $\mathscr{H} \subseteq \mathscr{L}$ such that

$$
\left(h_{i}, d_{j}\right)=0,\left(d_{j}, d_{k}\right)=0, \quad\left(h_{\delta_{k}}, d_{j}\right)=\delta_{k j},
$$

for $1 \leq i \leq 2,1 \leq j, k \leq \nu$. Letting $\mathscr{D}_{0}=\bigoplus_{j=1}^{\nu} \mathbb{C} d_{j}$ we have since $\left[\mathscr{L}_{\alpha}, \mathscr{L}_{-\alpha}\right]$ $=\left(\mathscr{L}_{\alpha}, \mathscr{L}_{-\alpha}\right) h_{\alpha}$ for all $\alpha \in R$ that $\mathscr{D}_{0} \cap[\mathscr{L}, \mathscr{L}]=(0)$ and as $h_{1}, h_{2}, h_{\delta_{1}}$, 
$\ldots, h_{\delta_{\nu}}, d_{1}, \ldots, d_{\nu}$ are linearly independent then $\operatorname{dim} \mathscr{H} \geq 2 \nu+2$. Moreover, the above notation for $d_{j}$ is justified because if $\alpha=\sum_{i=1}^{2} n_{i} \alpha_{i}+\sum_{j=1}^{\nu} m_{j} \delta_{j}$ then

$$
\left[d_{j}, x\right]=m_{j} x \text {, for all } x \in \mathscr{L}_{\alpha}, 1 \leq j \leq \nu .
$$

That is,$d_{j}$ is just the $j^{\text {th }}$ degree derivation, so we have

$$
\left[d_{j}, x\right]=\left(d_{j}, h_{\alpha}\right) x=\alpha\left(d_{j}\right) x, \text { for } x \in \mathscr{L}_{\alpha} .
$$

As in [BGK] one defines the core of $\mathscr{L}$ as follows.

Definition 3.22. (i) The core of $\mathscr{L}$, denoted $\mathscr{L}_{c}$, is the subalgbera of $\mathscr{L}$ generated by the non-isotropic root spaces $\mathscr{L}_{\alpha}$ for $\alpha \in R \backslash \Lambda$.

(ii) We say $\mathscr{L}$ is tame if the orthogonal complement of the core is just the center of the core. That is, $\mathscr{L}$ is tame if and only if $\mathscr{L}_{c}^{\perp}=\mathscr{Z}\left(\mathscr{L}_{c}\right)$.

(iii) If $\mathscr{L}$ is an elliptic irreducible tame quasi-simple Lie algebra we just say $\mathscr{L}$ is a $Q S$ Lie algebra.

Remark 3.23. It is easy to see that the core, $\mathscr{L}_{c}$, is an ideal of $\mathscr{L}$ as in [BGK] and if $\rho: \mathscr{L} \rightarrow \operatorname{End}\left(\mathscr{L}_{c}\right)$ denotes the representation afforded by the adjoint representation then one has $\operatorname{ker} \rho=C_{\mathscr{L}}\left(\mathscr{L}_{c}\right)=\mathscr{L}_{c}^{\perp}$ where $C_{\mathscr{L}}\left(\mathscr{L}_{c}\right)$ is the centralizer of the core in $\mathscr{L}$. Thus, $\mathscr{L}$ is tame if and only if

$$
\mathscr{Z}\left(\mathscr{L}_{c}\right)=\operatorname{ker} \rho=C_{\mathscr{L}}\left(\mathscr{L}_{c}\right)=\mathscr{L}_{c}^{\perp} \text {. }
$$

Letting $Q(\Delta)=\mathbb{Z} \alpha_{1} \oplus \mathbb{Z} \alpha_{2}$ be the root lattice of our root system $\Delta$ it is clear from (3.17) that $\mathscr{L}$ and $\mathscr{L}_{c}$ are both graded by $Q(\Delta) \oplus \Lambda \cong \mathbb{Z}^{\nu+2}$. Moreover, $\mathscr{L}_{c} \cap \mathscr{H}$ has dimension $\nu+2$ with basis $h_{1}, h_{2}, h_{\delta_{1}}, \ldots, h_{\delta_{\nu}}$. If $\mathscr{L}$ is tame then the orthogonal complement of the span of $h_{1}, h_{2}, h_{\delta_{1}}, \ldots, h_{\delta_{\nu}}, d_{1}, \ldots, d_{\nu}$ in $\mathscr{H}$ would be in $\mathscr{L}_{c}^{\perp}=\mathscr{Z}\left(\mathscr{L}_{c}\right)=$ the span of $h_{\delta_{1}}, \ldots, h_{\delta_{2}}$ and hence is zero so $\mathscr{L}$ tame implies $\operatorname{dim} \mathscr{H}=2+2 \nu$. It is clear also that $\dot{\mathfrak{g}}=s l_{3}(\mathbb{C})$ is contained in $\mathscr{L}_{c}$ as $s l_{3}(\mathbb{C})$ is generated by the root spaces $\mathscr{L}_{ \pm \alpha_{1}}, \mathscr{L}_{ \pm \alpha_{2}}$. Moreover, letting $\left(\mathscr{L}_{c}\right)^{\alpha}=\mathscr{L}_{c} \cap \mathscr{L}^{\alpha}$ where $\mathscr{L}^{\alpha}=\sum_{\delta \in \Lambda} \mathscr{L}_{\alpha+\delta}$ for all $\alpha \in \Delta$ then we have $\mathscr{L}_{c}=$ $\bigoplus_{\alpha \in \Delta}\left(\mathscr{L}_{c}\right)^{\alpha}$ is a Lie algebra graded by $\Delta$ of type $A_{2}$ according to Definition 2.6. Thus, by Proposition 3.17 of [BM] there is an alternative algebra $S$ with identity over $\mathbb{C}$, and a surjective homomorphism $\varphi: s t_{3}(S) \rightarrow \mathscr{L}_{c}$ whose kernel is central. Using the notation from these taking any $\alpha \in \Delta \backslash\{0\}$ we can write $\mathscr{L}^{\alpha}=\left(\mathscr{L}_{c}\right)^{\alpha}=\sum_{\delta \in \Lambda} \mathscr{L}_{\alpha+\delta}$, so since we may write $\left(\mathscr{L}_{c}\right)^{\alpha}=e_{\alpha}(S)$ where $\left\{h_{1}, h_{2}, e_{\alpha} \mid \alpha \in \Delta \backslash\{0\}\right\}$ is a Chevalley basis of $\dot{\mathfrak{g}}=s l_{3}(\mathbb{C})$ then we find we can let $S_{\delta}$ be the subspace of $S$ defined to satisfy

$$
e_{\alpha}\left(S_{\delta}\right)=\mathscr{L}_{\alpha+\delta} \subseteq \mathscr{L}_{c}, \text { for } \alpha \in \Delta \backslash\{0\}, \delta \in \Lambda .
$$

This does not depend on the choice of $\alpha$ and this can be seen exactly as one sees in [BM] that $S$ does not depend on $\alpha$. Our next result determines $S$. Note that we don't need the tameness assumption for this result which is about the core.

Lemma 3.25. Let $\mathscr{L}_{c}$ be the core of a QS algebra of type $A_{2}$ and let $S=\bigoplus_{\delta \in \Lambda} S_{\delta}$ be the coordinates as above. Then $S$ satisfies (1.4)-(1.7). Thus, either $S$ is a quantum torus $\mathbb{C}_{\mathbf{q}}$, for some $\nu \times \nu$ matrix $\mathbf{q}$ satisfying (1.1) or $S$ is the alternative torus $\mathfrak{A}=\mathfrak{A}\left(K, t_{1}, t_{2}, t_{3}\right)$ of Definition 1.21 where $K=\mathbb{C}\left[t_{1}^{ \pm 1}, \ldots, t_{\nu}^{ \pm 1}\right]$ and $\nu \geq 3$ is the nullity of $\mathscr{L}$.

Proof. Obviously since $\mathscr{L}^{\alpha}=\bigoplus_{\delta \in \Lambda} \mathscr{L}_{\alpha+\delta}$ for any $\alpha \in \Delta \backslash\{0\}$ we have $S=$ $\bigoplus_{\delta \in \Lambda} S_{\delta}$. Moreover, Theorem 3.11(i) yields $\operatorname{dim} S_{\delta}=1$ for all $\delta \in \Lambda$ and 
since $\left[\mathscr{L}_{\alpha+\delta}, \mathscr{L}_{\beta+\lambda}\right] \subseteq \mathscr{L}_{(\alpha+\beta)+(\delta+\lambda)}$ for $\alpha, \beta \in \Delta \backslash\{0\}$ with $\alpha+\beta \in \Delta \backslash\{0\}$ and any $\delta, \lambda \in \Lambda$ then it follows that $S_{\delta} S_{\lambda} \subseteq S_{\delta+\lambda}$. Also it is clear that $S_{0}=\mathbb{C} \cdot 1$ where 1 is the identity element of $S$ so we need only show $S_{\delta} S_{\lambda}=S_{\delta+\lambda}$, for $\delta, \lambda \in \Lambda$. Interpreting this on the level of root spaces we need to show if $\alpha, \beta \in$ $\Delta \backslash\{0\}$ and $\alpha+\beta \in \Delta \backslash\{0\}$ then for any $\delta, \lambda \in \Lambda$ we have $\left[\mathscr{L}_{\alpha+\delta}, \mathscr{L}_{\beta+\lambda}\right]=$ $\mathscr{L}_{(\alpha+\beta)+\delta+\lambda}$. Because non-isotropic root spaces are 1 -dimensional it is enough to show $\left[\mathscr{L}_{\alpha+\delta}, \mathscr{L}_{\beta+\lambda}\right] \neq(0)$. Using the Weyl group $W$ of $R=\Delta \times \Lambda$ we might as well assume that $\delta=0$ so that $\mathscr{L}_{\alpha+\delta}=\mathscr{L}_{\alpha}=\mathbb{C} e_{\alpha}(1)$ in the notation of [BM] while $\mathscr{L}_{\beta+\lambda}=e_{\beta}\left(S_{\lambda}\right)$. Take $0 \neq a_{\lambda} \in S_{\lambda}$ and then we have for

$$
n_{\alpha}=\exp \left(\operatorname{ad} e_{\alpha}\right) \exp \left(\operatorname{ad}\left(-e_{-\alpha}\right)\right) \exp \left(\operatorname{ad} e_{\alpha}\right) \in \operatorname{Aut}(\mathscr{L})
$$

that $\left(n_{\alpha} e_{\beta}\right)\left(a_{\lambda}\right)=n_{\alpha}\left(e_{\beta}\left(a_{\lambda}\right)\right)$ by Lemma 1.15 of [BM], so that $\left(n_{\alpha} e_{\beta}\right)\left(a_{\lambda}\right) \neq 0$ in $\mathscr{L}_{\alpha+\beta+\lambda}$ as $\alpha+\beta \in \Delta \backslash\{0\}$. This implies that ad $e_{\alpha}\left(e_{\beta}\left(a_{\lambda}\right)\right) \neq 0$, and hence that $\left[\mathscr{L}_{\alpha}, \mathscr{L}_{\beta+\lambda}\right] \neq(0)$ as desired. Lemma 1.8 and Corollary 1.26 now give our result.

When the coordinates of $\mathscr{L}$, as above, are the quantum torus the analysis proceeds as in [BGK] and we say no more about this case here. Thus, we assume that the coordinates are alternative but not associative. Then we have

Corollary 3.26. If $\mathscr{L}_{c}$ is as in Lemma 3.25 with coordinates which are not associative, then there is a subset $E$ of $H C_{1}(\mathfrak{A})$ where $\mathfrak{A}$ is the alternative torus such that $\mathscr{L}_{c}$ is isomorphic to the algebra $\mathfrak{g}(\mathfrak{A}, E)$ of Remark 2.8. Here $E \subseteq \bigoplus_{\mathbf{a} \in \mathbb{Z}^{\nu} \backslash\{0\}} H C_{1}(\mathfrak{A})_{\mathbf{a}}$.

Proof. We know that $\left(\mathscr{L}_{c}\right)^{0}$ is of dimension $2+\nu$ where $\nu$ is the nullity and the elements $h_{\delta_{1}}, \ldots, h_{\delta_{\nu}}$ are central in $\mathscr{L}_{c}$ as $\delta_{j} \in \Lambda, 1 \leq j \leq \nu$. The rest is clear by Theorem 2.17 .

Example 3.27. Let $\mathscr{D}^{\prime}$ be a graded subalgebra of $\tilde{D}$ containing $\mathscr{D}_{0}$ and let $\tau: \mathscr{D}^{\prime \prime} \times \mathscr{D}^{\prime \prime} \rightarrow \mathscr{Z}\left(\mathscr{D}^{\prime}\right)$ be any homogeneous 2-cocycle of invariant type as in Corollary 2.68. We know $L=L\left(\mathscr{D}^{\prime}, \tau\right)=\mathfrak{g}\left(\mathfrak{A}, \mathscr{E}\left(\mathscr{D}^{\prime}\right)\right) \oplus \mathscr{D}^{\prime}$ has an invariant symmetric non-degenerate bilinear form $F$. As $\mathfrak{g}\left(\mathfrak{A}, \mathscr{E}\left(\mathscr{D}^{\prime}\right)\right)$ is a homomorphic image of $s t_{3}(\mathfrak{A})$ we write $\{a, b\} \in \mathfrak{g}\left(\mathfrak{A}, \mathscr{E}\left(\mathscr{D}^{\prime}\right)\right)$ for the image of $\langle a, b\rangle \in s t_{3}(\mathfrak{A})$ under this natural map with kernel $\mathscr{E}\left(\mathscr{D}^{\prime}\right)$. Thus, $H C_{1}(\mathfrak{A}) / \mathscr{E}\left(\mathscr{D}^{\prime}\right)=\bigoplus_{\mathfrak{a} \in \mathbb{Z}^{\nu} \backslash \Gamma}\{\mathfrak{A}, \mathfrak{A}\}_{\mathfrak{a}}$ and so we have

$$
L\left(\mathscr{D}^{\prime}, \tau\right)=H C_{1}(\mathfrak{A}) / \mathscr{E}\left(\mathscr{D}^{\prime}\right) \oplus\left(\bigoplus_{\mathbf{a} \in \mathbb{Z}^{\nu} \backslash \Gamma}\{\mathfrak{A}, \mathfrak{A}\}_{\mathbf{a}}\right) \oplus\left(p s l_{3}(\mathbb{C}) \otimes \mathfrak{A}\right) \oplus \mathscr{D}^{\prime},
$$

with multiplication as in Section 2. One lets $\mathscr{H}=\mathscr{H}\left(\mathscr{D}^{\prime}, \tau\right)$ be the space spanned by the elements $h_{1} \otimes 1, h_{2} \otimes 1,\left\{t_{j}^{-1}, t_{j}\right\}, d_{j}, 1 \leq j \leq \nu$ so that $\mathscr{H}$ is abelian of dimension $2+\nu$. Notice that the form is non-degenerate on $\mathscr{H}$ and define $\delta_{1}, \ldots, \delta_{\nu} \in \mathscr{H}^{*}$ by saying $h_{\delta_{j}}=-\frac{1}{3}\left\{t_{j}^{-1}, t_{j}\right\} \in H C_{1}(\mathfrak{A}) / \mathscr{E}\left(\mathscr{D}^{\prime}\right)$. Then (2.56) gives us that

$$
F\left(d_{j}, h_{\delta_{k}}\right)=\delta_{j k}, \text { for } 1 \leq j, k \leq \nu .
$$

Letting $R=\Delta \times \Lambda \subseteq \mathscr{L}^{*}$ where $\Lambda=\mathbb{Z} \delta_{1} \oplus \cdots \oplus \mathbb{Z} \delta_{\nu}$ we have easily that $\mathscr{L}=\bigoplus_{\alpha \in R} \mathscr{L}_{\alpha}$. Indeed, if $\alpha=\beta+\delta$ where $\beta \in \Delta \backslash\{0\}, \lambda \in \Lambda$ then $\mathscr{L}_{\alpha}=$ $s l_{3}(\mathbb{C}) \times \mathfrak{A}_{\lambda}$, while if $\alpha=\lambda$ for $\lambda \in \Lambda$ then $\mathscr{L}_{\alpha}=\{\mathfrak{A}, \mathfrak{A}\}_{\lambda} \oplus \mathscr{D}_{\lambda}^{\prime} \oplus\left(\dot{\mathfrak{h}} \otimes \mathbb{C} t^{\lambda}\right)$ if 
$\lambda \in \Gamma \backslash\{0\}$. Thus, (3.3) and (3.4) hold. Certainly $R$ is discrete and (3.8) and (3.9) hold. The associated finite root system is just $\Delta$ so $\mathscr{L}$ is irreducible and clearly the transferred form to the real span of the roots (this is just the real span of $h_{\alpha_{1}}, h_{\alpha_{2}}, h_{\delta_{1}}, \ldots, h_{\delta_{\nu}}$ ) is positive semi-definite so $\mathscr{L}$ is irreducible of elliptic type. The core of $\mathscr{L}$ is just $\mathscr{L}_{c}=\mathfrak{g}\left(\mathfrak{A}, \mathscr{E}\left(\mathscr{D}^{\prime}\right)\right)$ so that $\mathscr{L}_{c}^{\perp}=$ $H C_{1}(\mathfrak{A}) / \mathscr{E}\left(\mathscr{D}^{\prime}\right)$ which is the center of $\mathscr{L}_{c}$ so $\mathscr{L}$ is tame. Thus, $L\left(\mathscr{D}^{\prime}, \tau\right)$ is a QS algebra according to Definition 3.22(iii).

We now want to show that any QS algebra is isomorphic to $L\left(\mathscr{D}^{\prime}, \tau\right)$ for some subalgebra $\mathscr{D}^{\prime}$ of $\tilde{\mathscr{D}}$ containing $\mathscr{D}_{0}$ and some homogeneous cocycle $\tau$ of invariant type. The arguments here are nearly like those of [BGK] (see (3.23) through (3.30) in [BGK]), but we repeat them because there are slight changes.

To begin we let $\mathscr{L}$ be a QS algebra so its core, $\mathscr{L}_{c}$, can be identified as in Corollary 3.26. In particular by Proposition 2.25 we have $V\left(\mathscr{L}_{c}\right) \cong \mathfrak{A} / \mathscr{I}(\mathfrak{A})$ so the restriction of the form, $F$, of $\mathscr{L}$ to $\mathscr{L}_{c}$ is given by $F_{\varphi}$ as in Remark 2.27 for some $\varphi \in V\left(\mathscr{L}_{c}\right)^{*}$. Now the degree derivations $d_{1}, \ldots, d_{\nu}$ are in the QS algebra $\mathscr{L}$ and so act as skew-symmetric derivations related to $F_{\varphi}$. Thus, $\varphi\left(d_{i}\left(g^{\mathbf{a}}\right)+\mathscr{I}(\mathfrak{A})\right)=0$ for any $\mathbf{a} \in \mathbb{Z}^{\nu}, 1 \leq i \leq \nu$. It follows that if we identify $\varphi$ with an element in $\mathfrak{A}^{*}$ which vanishes on $\mathscr{F}(\mathfrak{A})$ then $\varphi\left(g^{\mathfrak{a}}\right)=0$ for all $\mathbf{a} \in \mathbb{Z}^{\nu} \backslash\{0\}$ and hence $\varphi$ is a multiple of $\epsilon$ so that $F_{\varphi}$ is a non-zero multiple of our usual form $F$. Thus, we can now assume that

(3.30) the restriction of the form $F$ on $\mathscr{L}_{c}$ agrees with our previous form $F_{\varepsilon}$.

Recalling that $R=\Delta \times \Lambda$ is the root system of $\mathscr{L}$ let $0 \neq \delta=m_{1} \delta_{1}+\cdots+$ $m_{\nu} \delta_{\nu} \in R$ be isotropic and let $\mathbf{m}=\left(m_{1}, \ldots, m_{\nu}\right) \in \mathbb{Z}^{\nu}$. Suppose first that $\mathbf{m} \notin \Gamma$. Then we have (writing $\{\mathfrak{A}, \mathfrak{A}\}_{\delta}$ for $\{\mathfrak{A}, \mathfrak{A}\}_{\mathbf{m}}$ ) that

$$
\left\{\begin{array}{l}
\left(\mathscr{L}_{c}\right)_{\delta}=\{\mathfrak{A}, \mathfrak{A}\}_{\delta} \oplus\left(\dot{\mathfrak{h}} \otimes g^{\mathbf{m}}\right), \\
\left(\mathscr{L}_{c}\right)_{-\delta}=\{\mathfrak{A}, \mathfrak{A}\}_{-\delta} \oplus\left(\mathfrak{h} \otimes\left(g^{\mathbf{m}}\right)^{-1}\right) .
\end{array}\right.
$$

Moreover, the form is non-degenerate on $\{\mathfrak{A}, \mathfrak{A}\}_{\delta} \oplus\{\mathfrak{A}, \mathfrak{A}\}_{-\delta}$ as well as on $\left(\dot{\mathfrak{h}} \otimes g^{\mathbf{m}}\right) \oplus\left(\dot{\mathfrak{h}} \otimes\left(g^{\mathbf{m}}\right)^{-1}\right)$, and these two spaces are orthogonal as we see from (2.36)-(2.39). Letting $M_{ \pm \delta}=\left(\left(\mathscr{L}_{c}\right)_{\mp \delta}\right)^{\perp} \cap \mathscr{L}_{ \pm \delta}$ we see that the form is nondegenerate on $M_{\delta} \oplus M_{-\delta}$ and we have

$$
\left\{\begin{array}{l}
\mathscr{L}_{\delta}=\{\mathfrak{A}, \mathfrak{A}\}_{\delta} \oplus\left(\dot{\mathfrak{h}} \otimes g^{\mathbf{m}}\right) \oplus M_{\delta} \\
\mathscr{L}_{-\delta}=\{\mathfrak{A}, \mathfrak{A}\}_{-\delta} \oplus\left(\dot{\mathfrak{h}} \otimes\left(g^{\mathbf{m}}\right)^{-1}\right) \oplus M_{-\delta}
\end{array}\right.
$$

Because $\left(\mathscr{L}_{\alpha}, \mathscr{L}_{\beta}\right)=0$ if $\alpha+\beta \neq 0$ we obtain that $M_{\delta} \oplus M_{-\delta} \subseteq\left(\mathscr{L}_{c}\right)^{\perp}=$ $\operatorname{ker} \rho=\mathscr{Z}\left(\mathscr{L}_{c}\right) \subseteq \mathscr{L}_{c}$ since $\mathscr{L}$ is tame. Thus, $M_{\delta} \oplus M_{-\delta}=(0)$, and if $\delta \notin \Gamma$ then

$$
\left\{\begin{array}{l}
\mathscr{L}_{\delta}=\left(\mathscr{L}_{c}\right)_{\delta}=\{\mathfrak{A}, \mathfrak{A}\}_{\delta} \oplus\left(\dot{\mathfrak{h}} \otimes g^{\mathbf{m}}\right) \\
\mathscr{L}_{-\delta}=\left(\mathscr{L}_{c}\right)_{-\delta}=\{\mathfrak{A}, \mathfrak{A}\}_{-\delta} \oplus\left(\dot{\mathfrak{h}} \otimes\left(g^{\mathbf{m}}\right)^{-1}\right)
\end{array}\right.
$$

Assume now that $\delta \in \Gamma$ (i.e., $\mathbf{m} \in \Gamma$ ). Then

$$
\left\{\begin{array}{l}
\left(\mathscr{L}_{c}\right)_{\delta}=\left(\dot{\mathfrak{h}} \otimes g^{\mathbf{m}}\right) \oplus\{\mathfrak{A}, \mathfrak{A}\}_{\mathbf{m}}, \\
\left(\mathscr{L}_{c}\right)_{-\delta}=\left(\dot{\mathfrak{h}} \otimes\left(g^{\mathbf{m}}\right)^{-1}\right) \oplus\{\mathfrak{A}, \mathfrak{A}\}_{-\mathbf{m}},
\end{array}\right.
$$

where $\left(\dot{\mathfrak{h}} \otimes g^{\mathbf{m}}\right) \oplus\left(\dot{\mathfrak{h}} \otimes\left(g^{\mathbf{m}}\right)^{-1}\right)$ is non-degenerate, and $\{\mathfrak{A}, \mathfrak{A}\}_{\mathbf{m}} \oplus\{\mathfrak{A}, \mathfrak{A}\}_{-\mathbf{m}}$ is the radical of the invariant form on $\left(\mathscr{L}_{c}\right)_{\delta} \oplus\left(\mathscr{L}_{c}\right)_{-\delta}$ as this space is central in 
$\mathscr{L}_{c}$. The restriction of our form on $\mathscr{L}_{\delta} \oplus \mathscr{L}_{-\delta}$ is non-degenerate so we can find isotropic subspaces $W_{ \pm \delta}, M_{ \pm \delta} \subseteq \mathscr{L}_{ \pm \delta}$ such that

$$
\left\{\begin{array}{l}
\mathscr{L}_{\delta}=\left(\mathscr{L}_{c}\right)_{\delta} \oplus W_{\delta} \oplus M_{\delta}, \\
\mathscr{L}_{-\delta}=\left(\mathscr{L}_{c}\right)_{-\delta} \oplus W_{-\delta} \oplus M_{-\delta},
\end{array}\right.
$$

where the spaces $\left(\dot{\mathfrak{h}} \otimes g^{\mathbf{m}}\right) \oplus\left(\dot{\mathfrak{h}} \otimes\left(g^{\mathbf{m}}\right)^{-1}\right),\{\mathfrak{A}, \mathfrak{A}\}_{\mathbf{m}} \oplus W_{-\delta},\{\mathfrak{A}, \mathfrak{A}\}_{-\mathbf{m}} \oplus W_{\delta}$, and $M_{\delta} \oplus M_{-\delta}$ are non-degenerate and orthogonal to one another. It follows that

$$
\operatorname{dim}\{\mathfrak{A}, \mathfrak{A}\}_{\mathbf{m}}=\operatorname{dim} W_{-\delta}, \operatorname{dim}\{\mathfrak{A}, \mathfrak{A}\}_{-\mathbf{m}}=\operatorname{dim} W_{\delta} .
$$

Moreover, we have that $\{\mathfrak{A}, \mathfrak{A}\}_{\mathbf{m}} \oplus M_{\delta}=\left(\left(\mathscr{L}_{c}\right)_{-\delta}\right)^{\perp} \cap \mathscr{L}_{\delta}$, and $\{\mathfrak{A}, \mathfrak{A}\}_{-\mathbf{m}} \oplus$ $M_{-\delta}=\left(\left(\mathscr{L}_{c}\right)_{\delta}\right)^{\perp} \cap \mathscr{L}_{-\delta}$, so that $M_{\delta} \oplus M_{-\delta} \subseteq \operatorname{ker} \rho=\mathscr{Z}\left(\mathscr{L}_{c}\right)$ and again we get $M_{\delta} \oplus M_{-\delta}=(0)$. Thus, for $\delta \in \Gamma$ we have

$$
\left\{\begin{array}{l}
\mathscr{L}_{\delta}=\left(\mathscr{L}_{c}\right)_{\delta} \oplus W_{\delta}=\left(\dot{\mathfrak{h}} \otimes g^{\mathbf{m}}\right) \oplus\{\mathfrak{A}, \mathfrak{A}\}_{\mathbf{m}} \oplus W_{\delta}, \\
\mathscr{L}_{-\delta}=\left(\mathscr{L}_{c}\right)_{-\delta} \oplus W_{-\delta}=\left(\dot{\mathfrak{h}} \otimes\left(g^{\mathbf{m}}\right)^{-1}\right) \oplus\{\mathfrak{A}, \mathfrak{A}\}_{-\mathbf{m}} \oplus W_{-\delta}
\end{array}\right.
$$

Also, we have already seen that

$$
\mathscr{L}_{0}=(\dot{\mathfrak{h}} \otimes 1) \oplus\{\mathfrak{A}, \mathfrak{A}\}_{0} \oplus \mathscr{D}_{0},
$$

where $\mathscr{D}_{0}$ is the span of $d_{1}, \ldots, d_{\nu}$.

For $\delta=m_{1} \delta_{1}+\cdots+m_{\nu} \delta_{\nu}$ as above it is clear that the elements of $W_{\delta}$ act as skew-symmetric derivations on $\mathscr{L}_{c}$ of degree $\delta$. Thus, if $w_{\delta} \in W_{\delta} \backslash\{0\}$, then $\rho\left(w_{\delta}\right)=\operatorname{ad}\left(h \otimes g^{\mathbf{m}}\right)+\Gamma_{\mathbf{m}, \alpha}$ for some $h \in \dot{\mathfrak{h}}, \alpha \in \mathbb{C}^{\nu}$, with $(\alpha \mid \mathbf{m})=0$. Just as in $[\mathrm{BGK}]$ we have the following result.

Lemma 3.36. $\rho\left(w_{\delta}\right) \in \tilde{\mathfrak{D}}_{\mathrm{m}}$, and $\left.\rho\right|_{W_{\delta}}: W_{\delta} \rightarrow \tilde{\mathfrak{D}}_{\mathbf{m}}$ is injective.

Proof. $W_{\delta}$ and $\dot{\mathfrak{h}} \otimes\left(g^{\mathbf{m}}\right)^{-1}$ are orthogonal so that (3.5) implies that $\rho\left(w_{\delta}\right)(\dot{\mathfrak{h}} \otimes$ $\left.\left(g^{\mathbf{m}}\right)^{-1}\right)=(0)$. But

$$
\begin{aligned}
\rho\left(w_{\delta}\right)\left(\dot{\mathfrak{h}} \otimes\left(g^{\mathbf{m}}\right)^{-1}\right) & =\left(\operatorname{ad}\left(h \otimes g^{\mathbf{m}}\right)+\Gamma_{\mathbf{m}, \alpha}\right)\left(\dot{\mathfrak{h}} \otimes\left(g^{\mathbf{m}}\right)^{-1}\right) \\
& =\operatorname{ad}\left(h \otimes g^{\mathbf{m}}\right)\left(\dot{\mathfrak{h}} \otimes\left(g^{\mathbf{m}}\right)^{-1}\right),
\end{aligned}
$$

since $\Gamma_{\mathbf{m}, \alpha}\left(\dot{h} \otimes\left(g^{\mathbf{m}}\right)^{-1}\right)=(0)$ because $(\alpha \mid \mathbf{m})=0$. This implies that $h \otimes g^{\mathbf{m}}$ is orthogonal to $\dot{\mathfrak{h}} \otimes\left(g^{\mathbf{m}}\right)^{-1}$ so that $h=0$ as desired.

That $\rho$ is $1-1$ on $W_{\delta}$ follows since $\operatorname{ker} \rho \subseteq \mathscr{L}_{c}$ and $W_{\delta} \cap \mathscr{L}_{c}=(0)$.

Letting $\mathscr{W}=\bigoplus_{\delta \in \Gamma} W_{\delta}$ where we take $W_{0}=\mathscr{L}_{0}$ and $\mathscr{W}^{\prime}=\bigoplus_{\delta \in \Gamma \backslash\{0\}} W_{\delta}$, we find that $\left.\rho\right|_{\mathscr{W}}$ is an injective map of $\mathscr{W}$ to a subspace of $\tilde{\mathscr{D}}$ which contains $\mathscr{D}_{0}$. Moreover, it is clear that the image $\rho(\mathscr{W})$ is in fact a subalgebra $\mathscr{D}^{\prime}$ of $\tilde{\mathscr{D}}$ containing $\mathscr{D}_{0}$ because the invariance of the form implies $\left[W_{\delta}, W_{\lambda}\right] \subseteq$ $W_{\delta+\lambda}+\{\mathfrak{A}, \mathfrak{A}\}_{\delta+\lambda}$ for $\delta, \lambda \in \Gamma$ and $\{\mathfrak{A}, \mathfrak{A}\}_{\delta+\lambda}$ is central in $\mathscr{L}_{c}$. We identify $\mathscr{W}$ with $\mathscr{D}^{\prime}$ and $\mathscr{W}^{\prime}$ with $\mathscr{D}^{\prime \prime}=\bigoplus_{\mathbf{a} \in \Gamma \backslash\{0\}} \mathscr{D}_{\mathbf{a}}^{\prime}$ and note that then as the form is non-degenerate on $\mathscr{L}$ that the center of $\mathscr{L}_{c}$, which is $\bigoplus_{\mathbf{a} \in \Gamma}\{\mathfrak{A}, \mathfrak{A}\}_{\mathfrak{a}}$, is nondegenerately paired with $\mathscr{D}^{\prime}$ and so can be identified with $H C_{1}(\mathfrak{A}) / \mathscr{E}\left(\mathscr{D}^{\prime}\right)$. 
Thus, $\mathscr{L}_{c} \cong \mathfrak{g}\left(\mathfrak{A}, \mathscr{E}\left(\mathscr{D}^{\prime}\right)\right)$ and there is a homogeneous 2-cocycle $\tau: \mathscr{D}^{\prime \prime} \times \mathscr{D}^{\prime \prime} \rightarrow$ $\mathscr{Z}\left(\mathscr{D}^{\prime}\right)$ of invariant type as in Definition 2.66 so that $\mathscr{L} \cong L\left(\mathscr{D}^{\prime}, \tau\right)$. Hence, we have our main result.

Theorem 3.37. Let $\mathscr{L}$ be any $Q S$ algebra of nullity $\nu$ and let $\mathscr{L}_{c}$ be its core. Assume that the coordinates of $\mathscr{L}_{c}$ are not associative. Then $\nu \geq 3$ and there is a subalgebra $\mathscr{D}^{\prime}$ of $\tilde{D}$ which contains $\mathscr{D}_{0}$ and a homogeneous 2-cocycle $\tau: \mathscr{D}^{\prime \prime} \times \mathscr{D}^{\prime \prime} \rightarrow \mathscr{Z}\left(\mathscr{D}^{\prime}\right)$ of invariant type such that $\mathscr{L} \cong L\left(\mathscr{D}^{\prime}, \tau\right)$. Moreover any of the algebras, $L\left(\mathscr{D}^{\prime}, \tau\right)$, is a $Q S$ algebra.

Note that we have the following bounds on root space dimensions:

$$
\begin{aligned}
& \text { if } \alpha \in R \text { is non-isotropic, } \operatorname{dim} \mathscr{L}_{\alpha}=1, \\
& \text { if } \alpha \in \Lambda \backslash \Gamma \text {, then } \operatorname{dim} \mathscr{L}_{\alpha}=2+2=4, \\
& \text { if } \alpha \in \Gamma \backslash\{0\}, \operatorname{then} 2 \leq \operatorname{dim} \mathscr{L}_{\alpha} \leq 2+2(\nu-1)=2 \nu, \\
& \text { if } \alpha=0 \text {, then } \operatorname{dim} \mathscr{L}_{0}=2+2 \nu .
\end{aligned}
$$

These bounds are sharp since $L_{\max }$ satisfies the upper bound while $L_{\min }$ satisfies the lower bound.

\section{REFERENCES}

[AF] B. N. Allison and J. R. Faulkner, Nonassociative coefficient algebras for Steinberg unitary Lie algebras, J. Algebra 161 (1993), 1-19.

[BGK] S. Berman, Y. Gao, and Y. Krylyuk, Quantum tori and the structure of elliptic quasi-simple Lie algebras, J. Funct. Anal. (to appear).

[BeK] S. Berman and Ya. S. Krylyuk, Universal central extensions of twisted and untwisted Lie algebras extended over commutative rings, J. Algebra (to appear).

[BM] S. Berman and R. V. Moody, Lie algebras graded by finite root systems and the intersection matrix algebras of Slodowy, Invent. Math 108 (1992), 323-347.

[BeM] G. M. Benkart and R. V. Moody, Derivations, central extensions and affine Lie algebras, Algebras, Groups and Geometries 3 (1986), 456-492.

[BZ] G. M. Benkart and E. I. Zelmanov, Lie algebras graded by finite root systems and intersection matrix algebras, Preprint.

[BK] H. Braun and M. Koecher, Jordan-Algebren, Springer-Verlag, Berlin, 1966.

[F] J. R. Faulkner, Barbilian planes, Geom. Dedicata 30 (1989), 125-181.

[G] Y. Gao, Skew-dihedral homology and involutive Lie algebras graded by finite root systems, Ph.D. Thesis, University of Saskatchewan, 1994.

[H-KT] R. Høegh-Krohn and B. Torresani, Classification and construction of quasi-simple Lie algebras, J. Funct. Anal. 89 (1990), 106-136.

[KL] C. Kassel and J. L. Loday, Extensions centrales d'algèbres de Lie, Ann. Inst. Fourier (Grenoble) 32 (1982), 119-142.

[M] Y. I. Manin, Topics in noncommutative geometry, Princeton University Press, 1991.

[McC] K. McCrimmon, Derivations and Cayley derivations of generalized Cayley-Dickson algebras, Pacific J. Math 117 (1985), 163-182.

[N] E. Neher, Lie algebras graded by 3-graded root systems and Jordan pairs covered by a grid, Preprint.

[S] R. D. Schafer, An introduction to nonassociative algebras, Academic Press, New York and London, 1966.

[Se] G. B. Seligman, Rational methods in Lie algebras, Lecture Notes Pure Appl. Math., Marcel Dekker, New York, 1976. 
[SSSZ] I. P. Shestakov, A. I. Shirshov, A. M. Slin'ko and K. A. Zhevlakov, Rings that are nearly associative, Academic Press, New York, 1982.

[W] M. Wambst, Homologie de Hochschild et homologie cyclique du tore quantique multipara métré, Preprint.

(Stephen Berman and Yun Gao) Department of Mathematics and Statistics, University of Saskatchewan, Saskatoon, Saskatchewan, Canada S7N 0W0

E-mail address: berman@snoopy. usask.ca

Department of Mathematics, Chernivtsi State University, Chernivtsi, Ukraine 274012

Department of Mathematics, University of Ottawa, Ottawa, Ontario, Canada K1N $6 \mathrm{~N} 5$

E-mail address: neher@acadvml.uottawa.ca 